\title{
Mild and selective mono-iodination of unprotected peptides as initial step for the synthesis of bioimaging probes
}

\author{
Romain Bertrand, ${ }^{\dagger}$ Michael Wagner, ${ }^{\dagger}$ Volker Derdau, ${ }^{\dagger, *}$ and Oliver Plettenburg ${ }^{\dagger, \ddagger, \S,{ }^{*}}$ \\ ${ }^{\dagger}$ Research \& Development, Integrated Drug Discovery, Sanofi-Aventis Deutschland GmbH, Frankfurt am Main, \\ 65926, Germany \\ ‡ Leibniz Universität Hannover, Schneiderberg 1B , 30167 Hannover, Germany \\ $\S$ Institute of Medicinal Chemistry, Helmholtz Zentrum München GmbH, German Research Center for \\ Environmental Health, Ingolstädter Landstr. 1, 85764 Neuherberg, Germany \\ Email: Volker.Derdau@sanofi.com; Oliver.Plettenburg@helmholtz-muenchen.de
}

\section{Supporting Information}

\section{$\underline{\text { Table of Contents }}$}

Figures S1 Preparation of the iodination stock solution (p.2)

Figures S2 lodination reaction occurs quickly at room temperature (p.3)

Figures S3 lodination is specific to tyrosine residue (p.4)

Figures S4 Mono-iodination of a methionine containing peptide: $\left[\right.$ Tyr $\left.^{8}\right]$-Substance P (p.7)

General methods (p.9)

Peptide mono-iodination (p.13)

NMR Spectra and proton assignment of mono-iodinated peptides ( $p .42$ )

Supporting References (p.70) 


\section{Figure S1 - lodination stock solution preparation}

lodination stock solution $50 \mathrm{mM}$ in acetonitrile was prepared freshly. Selectfluor (15 mg, $42.3 \mu \mathrm{mol}$ ) was dissolved in $840 \mu \mathrm{L}$ of acetonitrile by vigorous vortexing (solution A) followed by Nal (6.8 mg, $45.3 \mu \mathrm{mol}$ ). The addition of sodium iodide was easily visible by change in color: the transparent Selectfluor solution turned into a brown caramel mixture after 10 seconds (solution B).

Upon addition of the iodination stock solution to Tyr-peptides dissolved in DCM + 20\% TFA (solution C), the reaction mixture turned light pink (solution $\mathbf{D}$ ).
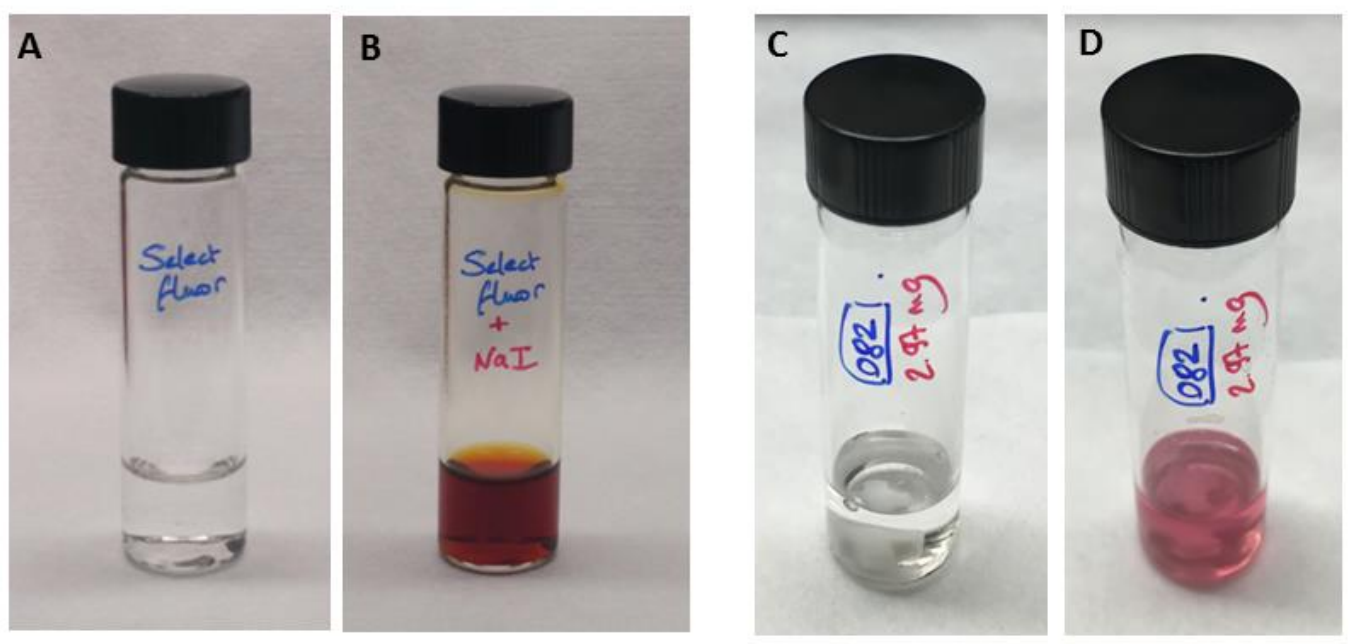


\section{Figure S2 - lodination reaction occurs quickly at room temperature}

Ac-Tyr-NH-Me $(2.0 \mathrm{mg}, 8.5 \mu \mathrm{mol})$ was dissolved in $1 \mathrm{~mL}$ of DCM $+10 \%$ TFA. Then was added dropwise 1.1 eq of a $50 \mathrm{mM}$ freshly prepared iodination stock solution. Reaction was allowed to stir at room temperature and monitored by LC-MS after 15 minutes and after 2 hours. No difference was observed. Analytical LC-MS chromatograms are shown below:
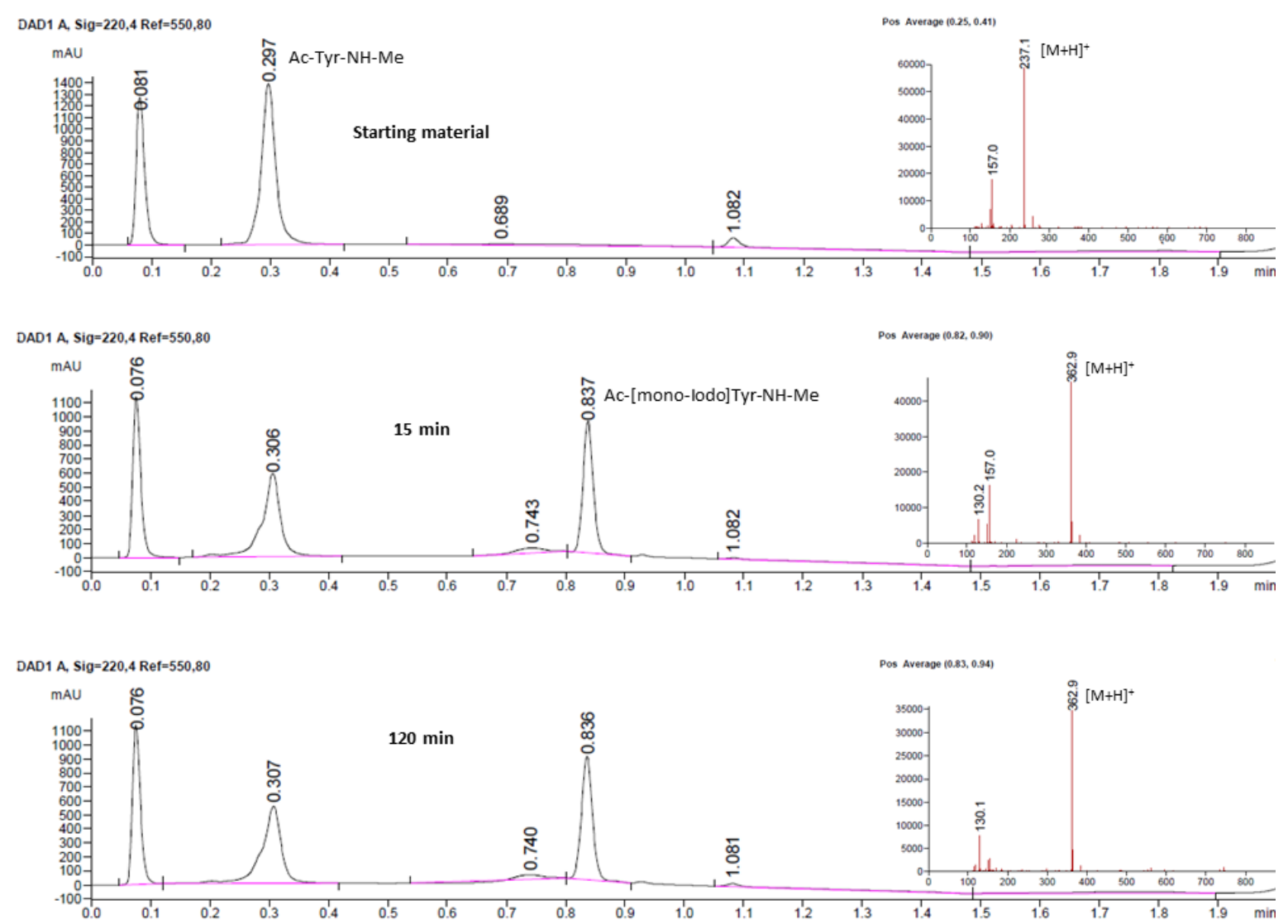


\section{Figure S3 - lodination is specific to Tyr residue}

Ac-Tyr-NH-Me $(2.0 \mathrm{mg}, 8.5 \mu \mathrm{mol})$, Ac-Phe-Tyr-NH-Me, Ac-Phe-His-NH-Me and Ac-Phe-Trp-NH-Me were respectively dissolved in $1 \mathrm{~mL}$ of DCM $+10 \%$ TFA. Then was added dropwise 1.1 eq of a $50 \mathrm{mM}$ freshly prepared iodination stock solution. Reaction was allowed to stir at room temperature and monitored by LC-MS after 2 minutes and after 15 minutes. Under these conditions, iodination proceeded only on Tyr residues. Analytical LC-MS chromatograms are shown below:
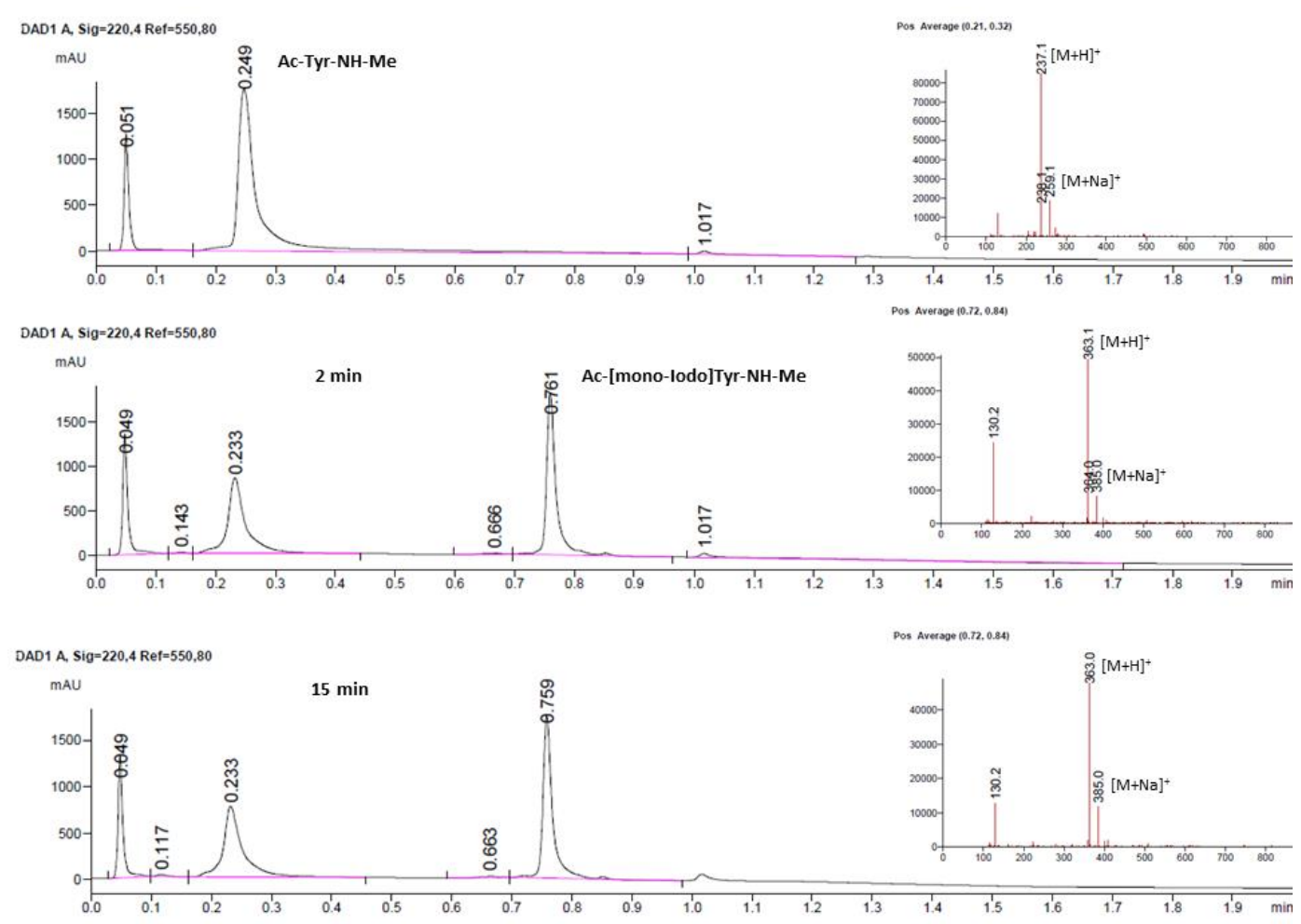

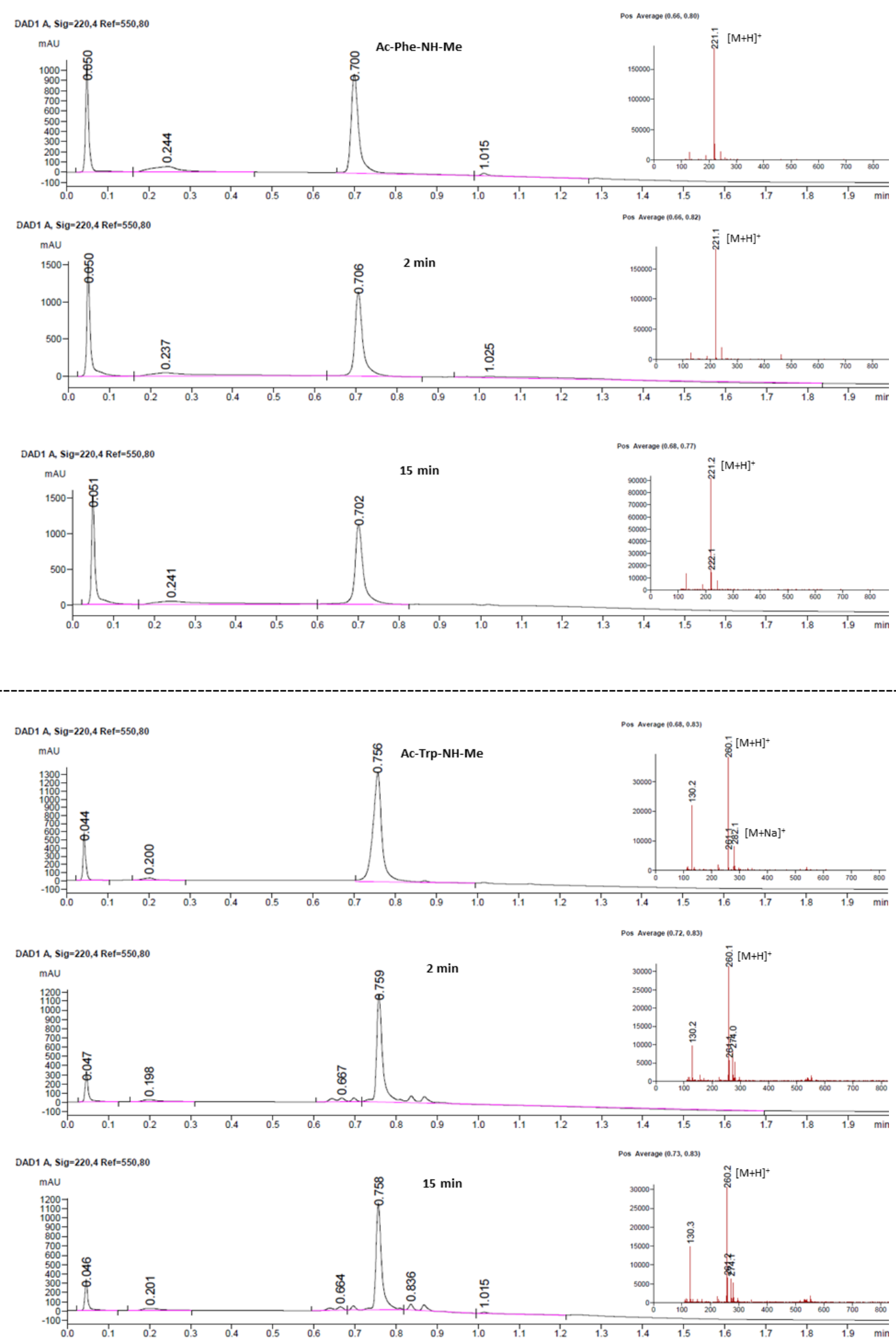

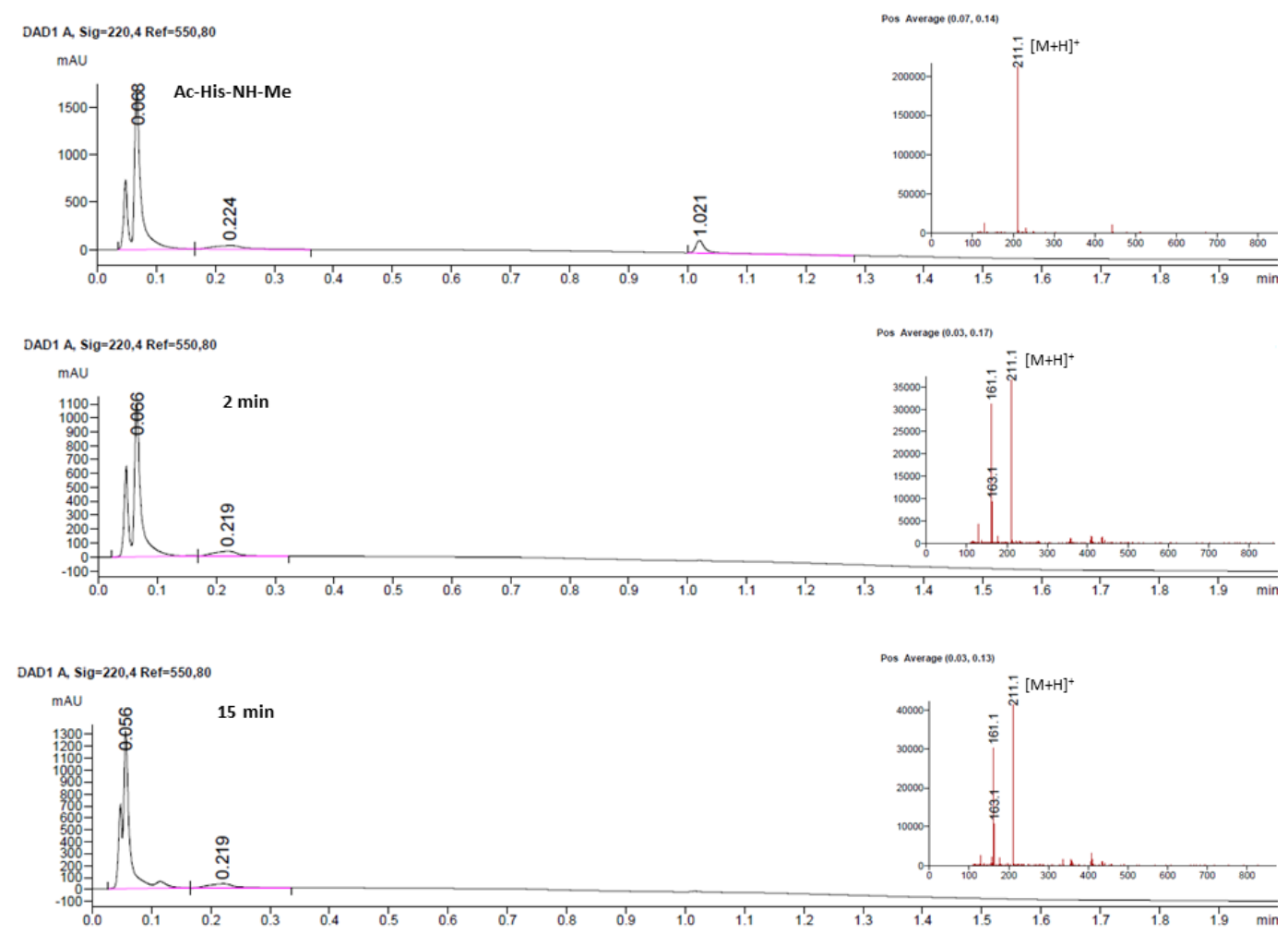
Figure S4 - Mono-iodination of a Met-containing peptide: [Tyr ${ }^{8}$ ]-Substance $\mathbf{P}$

$\left[\right.$ Tyr $^{8}$ ]-Substance $P(0.81 \mathrm{mg}, 0.59 \mu \mathrm{mol})$ was dissolved in $340 \mu \mathrm{L}$ of DCM and $80 \mu \mathrm{L}$ of TFA. Then were added dropwise $16 \mu \mathrm{L}$ (1.4 eq.) of a $50 \mathrm{mM}$ freshly prepared iodination stock solution. Reaction was allowed to stir at room temperature and monitored by LC-MS. A mixture containing the SM, the starting material with the oxidized methionine SM[Ox], and the MI. Addition of $4 \mu \mathrm{L}$ ( 0.35 eq.) of a $50 \mathrm{mM}$ iodination stock solution generated the oxidized methionine mono-iodinated compound $\mathrm{MI}[\mathrm{Ox}]$. Two other successive additions of $4 \mu \mathrm{L}$ ( $3 \times 0.35$ eq.) of a $50 \mathrm{mM}$ iodination stock solution afforded $\mathrm{MI}$ and $\mathrm{MI}[\mathrm{Ox}]$ as the major product. Finally, addition of $100 \mu \mathrm{L}$ of the reducing cocktail (freshly prepared: potassium iodide $\mathrm{KI}(10 \mathrm{mg})$ and ascorbic acid $(10 \mathrm{mg})$ were sonicated in $500 \mu \mathrm{L}$ of TFA for 10 minutes) enabled reduction of MI[Ox] towards MI. Analytical LC-MS chromatograms are shown below:
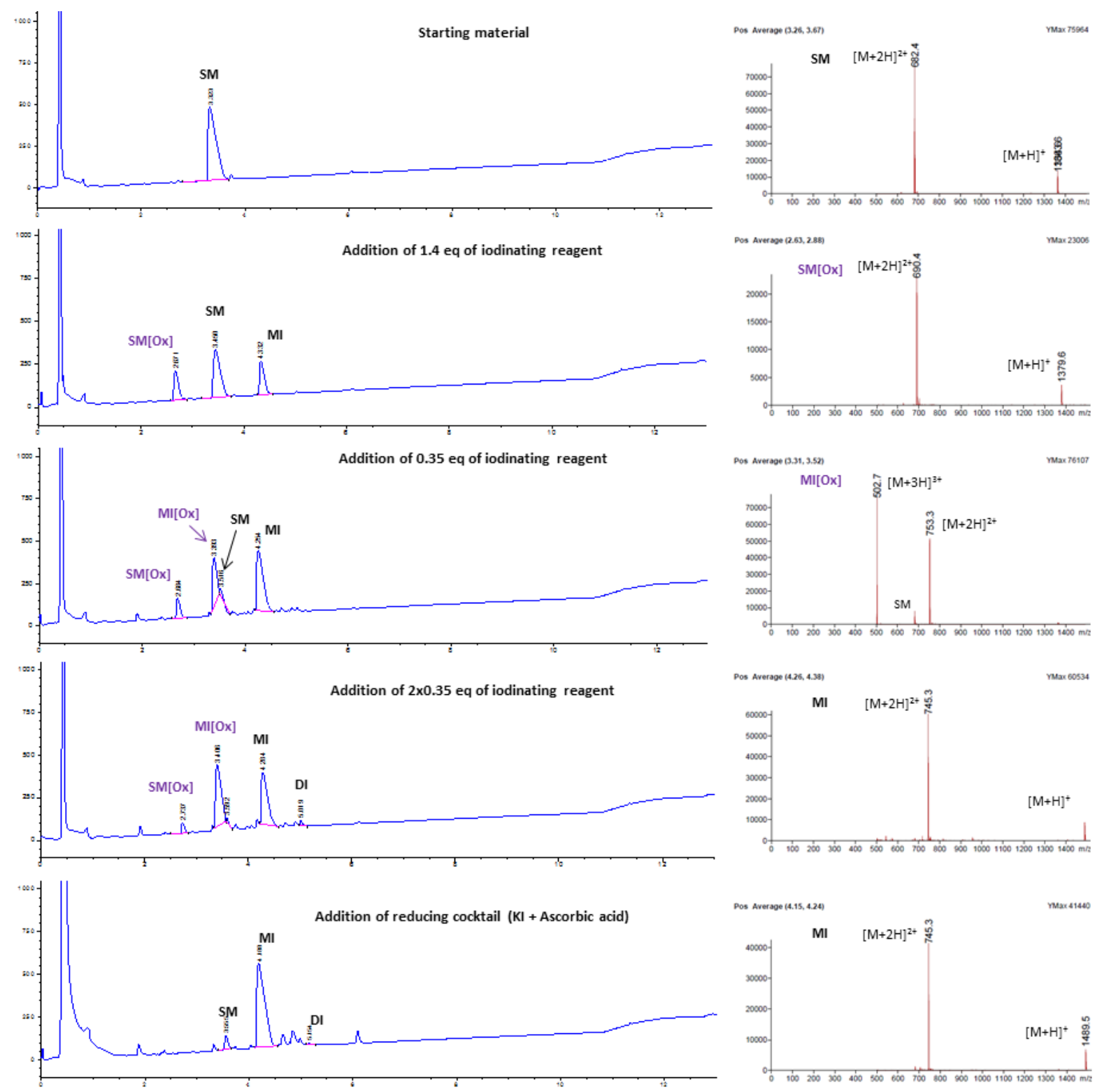

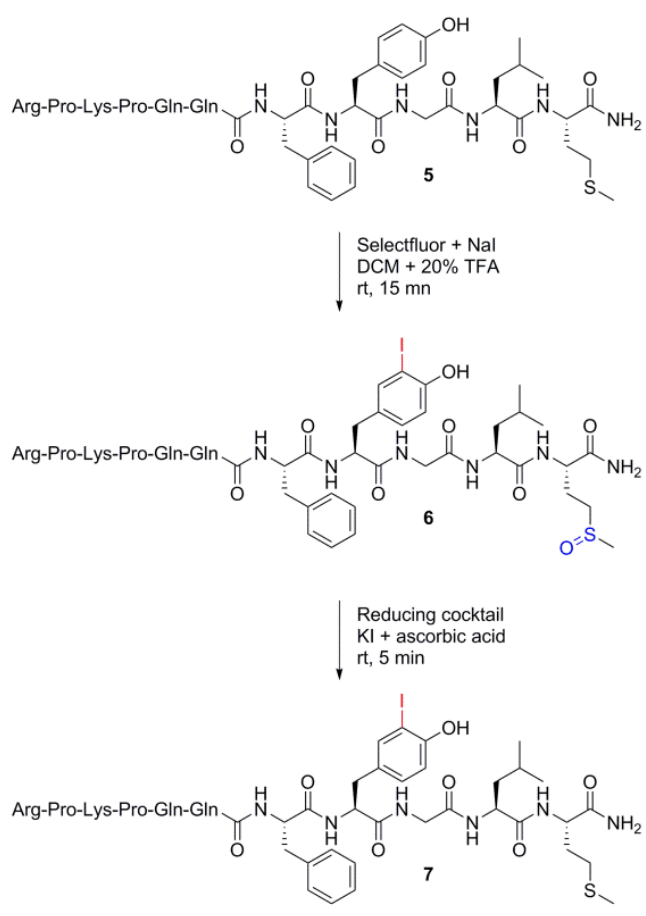

[Tyr ${ }^{8}$ ]-Substance $\mathbf{P}$

SM: $5.9 \%$ / MI: $93.6 \%$ / DI: 0.5\%

$2.2 \mu \mathrm{mol}$ - yield $72 \%$ 


\section{General methods}

Unless otherwise noted, all reagents and solvents were purchased from commercial suppliers (Sigma Aldrich, ThermoFisher, Merck Millipore) and used without further purification.

Reactions were monitored by LC-MS. For small molecules: data were acquired using the Agilent 1100 MSD system with a Phenomenex Luna column (C-18, 100Å pore size, $3 \mu \mathrm{m}$ particle size, $10 \times 2.0 \mathrm{~mm}$, flow: $1.1 \mathrm{~mL} / \mathrm{min})$. Gradient: 0 min 1\% ACN (+0.05\% TFA) / 99\% $\mathrm{H}_{2} \mathrm{O}$ (+0.05\% TFA); 0.3 min \% ACN (+0.05\% TFA); 1.3 min 95\% ACN (+0.05\% TFA); 1.75 min 1\% ACN (+0.05\% TFA); 1.80 min 1\% ACN (+0.05\% TFA). Mass detection range: $110-1000 \mathrm{MW}$. Temperature: $30^{\circ} \mathrm{C}$. For peptides: data were acquired using the Agilent 1100 MSD system with a Phenomenex Aeris Widepore column (XB-C18, 200Å pore size, $3.6 \mu \mathrm{m}$ particle size, $100 \times 2.1 \mathrm{~mm}$, flow: $0.5 \mathrm{~mL} / \mathrm{min}$ ). Gradient: 0 min 5\% ACN (+0.1\% formic acid) / 95\% $\mathrm{H}_{2} \mathrm{O}$ (+0.1\% formic acid) to $10 \mathrm{~min}-50 \% \mathrm{ACN}$ ( $+0.1 \%$ formic acid); $11 \mathrm{~min} 90 \% \mathrm{ACN}$ (+0.1\% formic acid) to $12.5 \mathrm{~min} ; 12.5 \mathrm{~min}$ to $13.5 \mathrm{~min} 5 \% \mathrm{ACN}$ (+0.1\% formic acid). Mass detection range: $500-1500 \mathrm{MW}$. Temperature: $38^{\circ} \mathrm{C}$.

Purifications on reverse-phase preparative HPLC were performed on the HP-Agilent 1100 with either i) a column from Agilent (Zorbax Rx C18, $5 \mu \mathrm{m}$ particle size, 250x9.4mm, flow: $4 \mathrm{~mL} / \mathrm{min}$ ). Gradient: $0 \mathrm{~min}$ to 5 min $10 \%$ ACN (+0.1\% TFA) / 90\% $\mathrm{H}_{2} \mathrm{O}\left(+0.1 \%\right.$ TFA); 5 min to 30 min 95\% ACN (+0.1\% TFA) / 5\% $\mathrm{H}_{2} \mathrm{O}$ (+0.1\% TFA); 30 min to 32 min 95\% ACN (+0.1\% TFA) / 5\% $\mathrm{H}_{2} \mathrm{O}(+0.1 \%$ TFA); 32 min to 35 min 10\% ACN $\left(+0.1 \%\right.$ TFA) $/ 90 \% \mathrm{H}_{2} \mathrm{O}(+0.1 \%$ TFA) or ii) a column from Waters (Xbridge Prep C18 OBD, $5 \mu \mathrm{m}$ particle size, 250x19mm, flow: $16 \mathrm{~mL} / \mathrm{min})$. Gradient: 0 min to $5 \mathrm{~min} 10 \% \mathrm{ACN}(+0.1 \% \mathrm{TFA}) / 90 \% \mathrm{H}_{2} \mathrm{O}(+0.1 \%$ TFA); 5 min to $30 \mathrm{~min} 95 \% \mathrm{ACN}\left(+0.1 \%\right.$ TFA) / 5\% $\mathrm{H}_{2} \mathrm{O}(+0.1 \%$ TFA); $30 \mathrm{~min}$ to $32 \mathrm{~min} 95 \% \mathrm{ACN}(+0.1 \%$ TFA) / 5\% $\mathrm{H}_{2} \mathrm{O}$ (+0.1\% TFA); 32 min to $35 \mathrm{~min} 10 \% \mathrm{ACN}(+0.1 \% \mathrm{TFA}) / 90 \% \mathrm{H}_{2} \mathrm{O}(+0.1 \% \mathrm{TFA})$ or iii) a

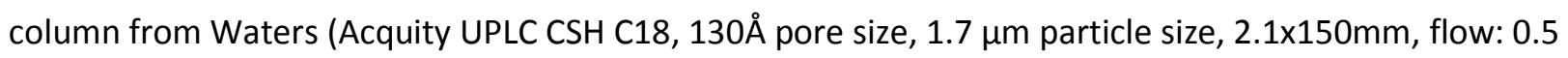
$\mathrm{mL} / \mathrm{min})$. Gradient: 0 min to $3 \mathrm{~min} 20 \% \mathrm{ACN}(+0.05 \% \mathrm{TFA}) / 80 \% \mathrm{H}_{2} \mathrm{O}(+0.05 \% \mathrm{TFA}) ; 3 \mathrm{~min}$ to $23 \mathrm{~min} 75 \%$ ACN (+0.05\% TFA) / 25\% $\mathrm{H}_{2} \mathrm{O}$ (+0.05\% TFA); $23 \mathrm{~min}$ to $23.5 \mathrm{~min} 95 \% \mathrm{ACN}(+0.05 \% \mathrm{TFA}) / 5 \% \mathrm{H}_{2} \mathrm{O}(+0.05 \%$ TFA). 23.5 min to 25.5 min 95\% ACN (+0.05\% TFA) / 5\% $\mathrm{H}_{2} \mathrm{O}$ (+0.05\% TFA). Temperature: $50{ }^{\circ} \mathrm{C}$. Purification on silica gel chromatography was performed on CombiFlash Rf-Isco Teledyne.

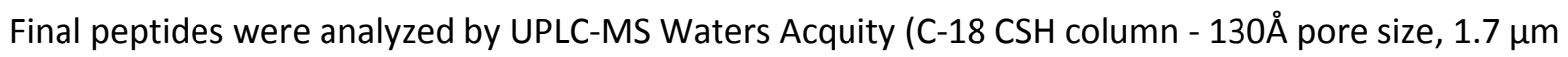
particle size, $150 \times 2.1 \mathrm{~mm}$, flow: $0.5 \mathrm{~mL} / \mathrm{min}$ - Gradient 1: 0 min 10\% ACN (+0.1\% formic acid) / $90 \% \mathrm{H}_{2} \mathrm{O}$ (+0.1\% formic acid) to $19.2 \mathrm{~min}-90 \% \mathrm{ACN}$ (+0.1\% formic acid); $20 \mathrm{~min} 90 \% \mathrm{ACN}(+0.1 \%$ formic acid). Gradient 2: 0 min 2\% ACN ( $+0.1 \%$ formic acid) / 98\% $\mathrm{H}_{2} \mathrm{O}$ (+0.1\% formic acid) to $9.12 \mathrm{~min}-40 \% \mathrm{ACN}$ (+0.1\% formic acid); 12 min 40\% ACN (+0.1\% formic acid). Mass detection range: $500-2000 \mathrm{MW}$.

Temperature: $40^{\circ} \mathrm{C}$. High resolution mass HRMS were recorded on the Agilent 6200 Series AccurateMass Time-of-flight (TOF). ${ }^{1} \mathrm{H}$ and ${ }^{13} \mathrm{C}$ NMR spectra were recorded on a Bruker DRX-400 or 600 systems in $\mathrm{d}_{6}$-DMSO or $\mathrm{CDCl}_{3}$. Chemical shifts are given in parts per million (ppm) with tetramethylsilane as an internal standard. Abbreviations are used as follows: $s=$ singlet, $d=$ doublet, $t=$ triplet, $q=$ quartet, $p=$ pentet, $\mathrm{m}=$ multiplet or unresolved, $\mathrm{dd}=$ doublets of doublet, $\mathrm{br}=$ broad. Coupling constants ( $J$ values) are given in Hertz $(\mathrm{Hz})$. Absorption and Emission spectra were acquired with a Thermo Varioskan using the Skanlt 2.4.3 software. 


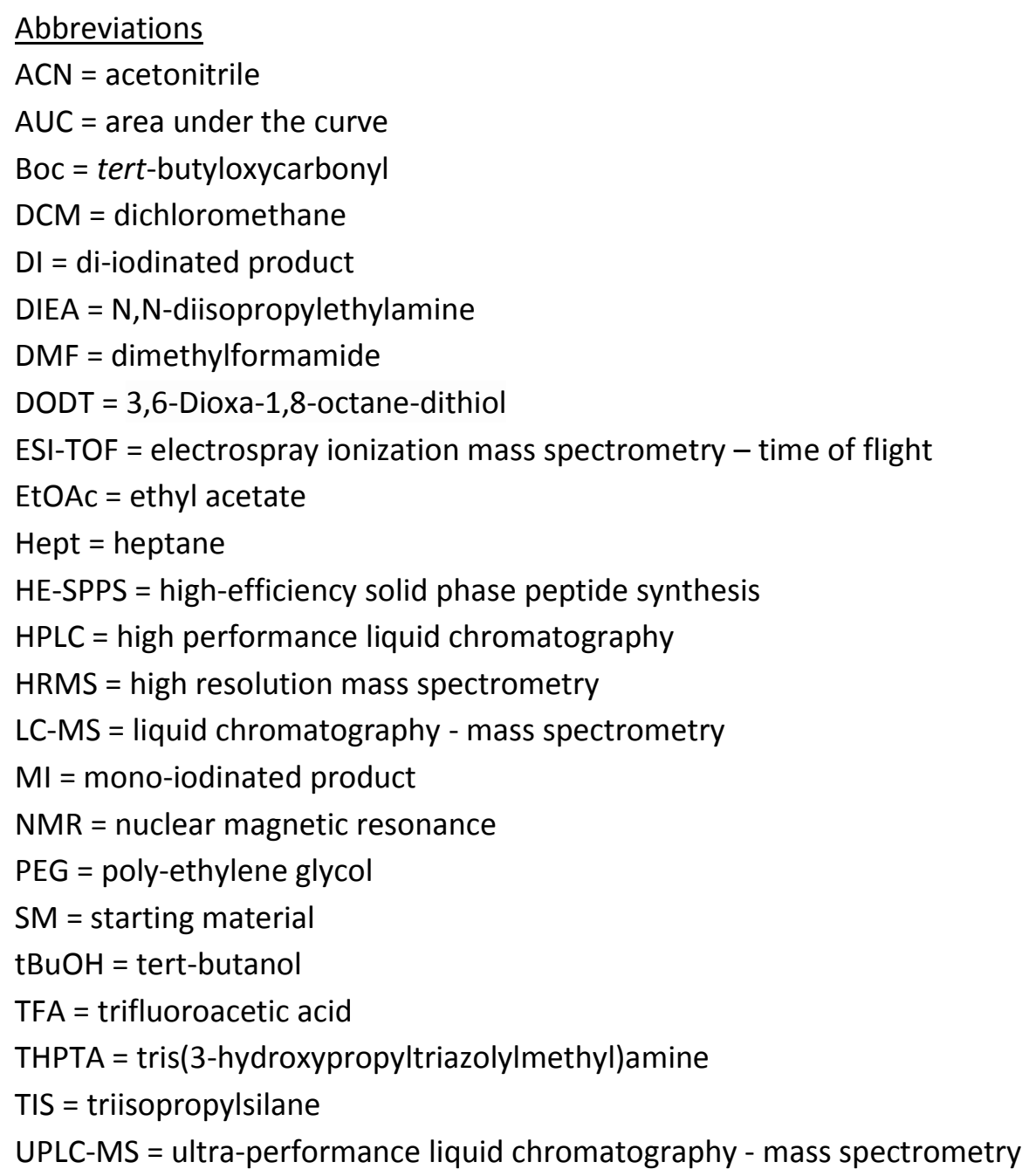

\section{Peptides}

Tocinoic acid, Goserelin acetate and $\left[\mathrm{Tyr}^{8}\right]$-Substance $\mathrm{P}$ were purchased from Sigma Aldrich.

Cyclo(RGGyK) was purchased from Selleckchem. AcMeYVAD-CHO, [Tyr $\left.{ }^{0}\right]$-Bradykinin, and human GLP-1 (7-37) were purchased from Bachem.

\section{$\underline{\text { HE-SPPS }}$}

Leucin-Enkephalinamide, Angiotensin III and ACP fragment (65-74) were synthesized by High-Efficiency Solid Phase Peptide Synthesis ${ }^{1}$ using a CEM Liberty Blue system on a $0.1 \mathrm{mmol}$ scale with a Rink Amide AM resin low loading $(0.29 \mathrm{mmol} / \mathrm{g})$ 100-200 mesh from Novabiochem using 5-fold excess of reagents [0.2 M Fmoc amino acid solution (in DMF) with $0.5 \mathrm{M} \mathrm{DIC} \mathrm{(in} \mathrm{DMF)} \mathrm{and} 1.0 \mathrm{M}$ Oxyma (in DMF)] and 20\% pipieridine in DMF for the Fmoc-deprotection cycles. Immediately after synthesis, the peptide resin was washed three times with $10 \mathrm{~mL}$ of DMF and then three times with $10 \mathrm{~mL}$ of DCM. Cleavage was then performed in all cases with $10 \mathrm{~mL}$ of a freshly prepared King's cocktail (TFA 82.5\% / Phenol 5\% / Thioanisol 5\% / $\mathrm{H}_{2} \mathrm{O} 5 \%$ / DODT 2.5\%) for 3 hours before being precipitated in $70 \mathrm{~mL}$ of ice cold diisopropyl ether. Precipitate was centrifuged (4 min, $4000 \mathrm{rpm}, 4{ }^{\circ} \mathrm{C}$ ) and washed with ice cold diisopropyl ether three times. Finally, the resin was filtered off and the peptide precipitate was dissolved 
in $\mathrm{H}_{2} \mathrm{O}+25 \% \mathrm{ACN}+0.5 \% \mathrm{AcOH}$ and lyophilized. Crude purity was > 95\% for Leucin-Enkephalinamide. Angiotensin III and ACP fragment (65-74) were purified with reverse-phase preparative HPLC.

\section{Solvent screening for Ac-Tyr-NH-Me mono-iodination}

Ac-Tyr-NH-Me $(2.0 \mathrm{mg}, 8.5 \mu \mathrm{mol})$ was dissolved in $1 \mathrm{~mL}$ of different solvents (indicated in Table 1). Then was added dropwise 1.1 eq of a $50 \mathrm{mM}$ freshly prepared iodination stock solution. Reaction was allowed to stir at room temperature and monitored by LC-MS instantly after addition of iodination solution, and after 15 minutes. Relative amounts of SM, MI, and DI shown in Table 1 were quantified using AUC integration (absorbance at $220 \mathrm{~nm}$ ). Analytical LC-MS chromatograms are shown below:
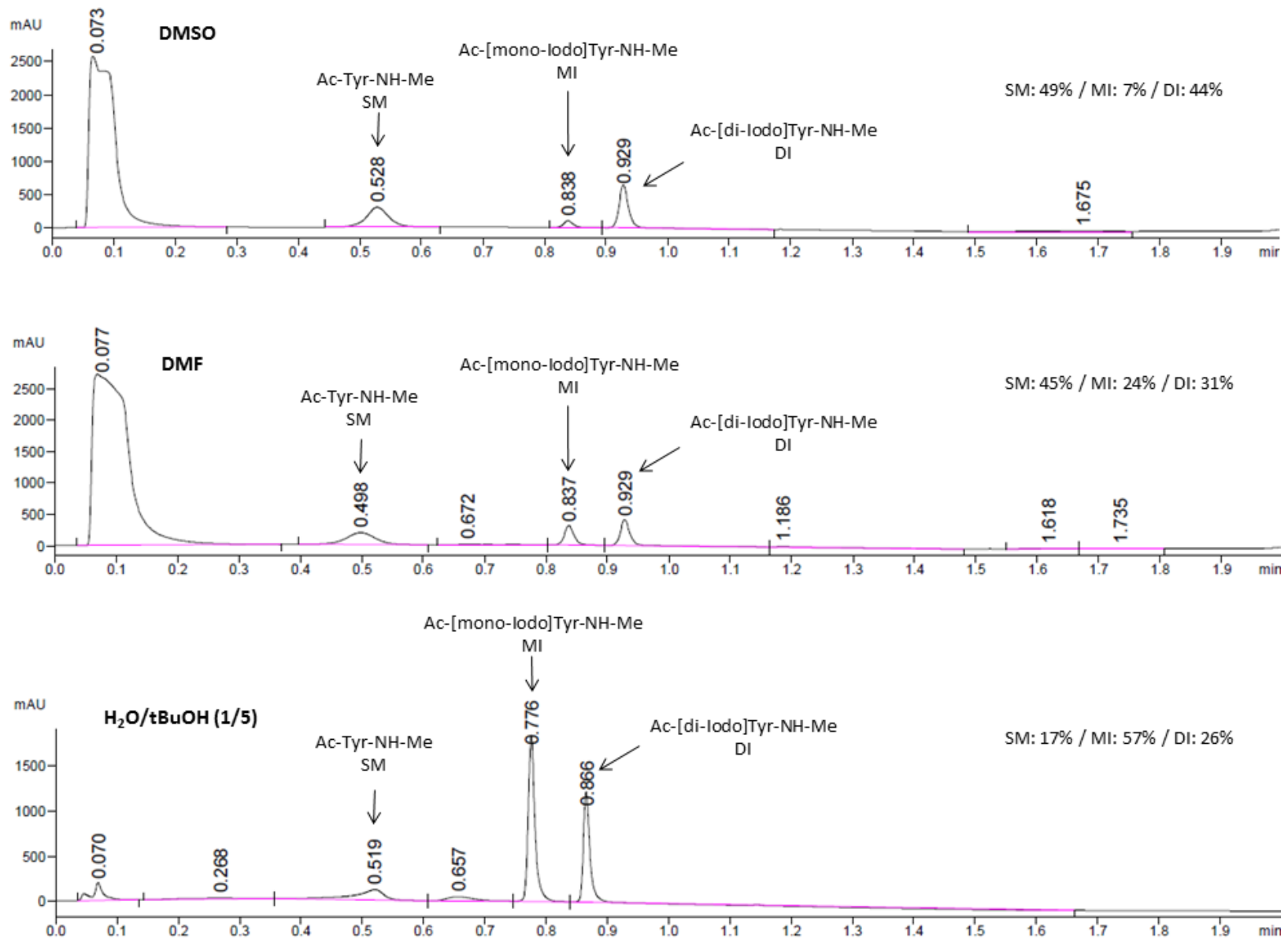

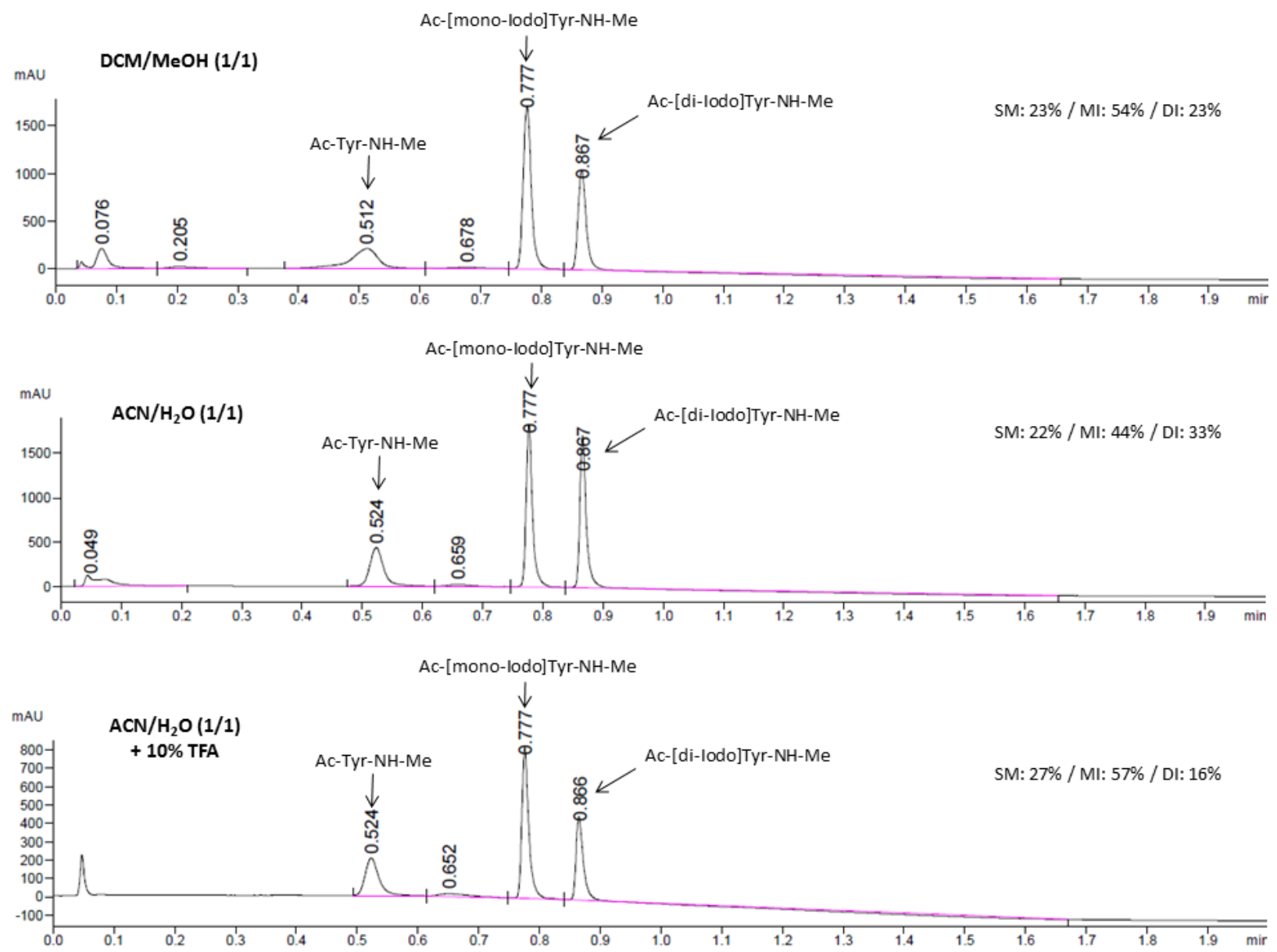

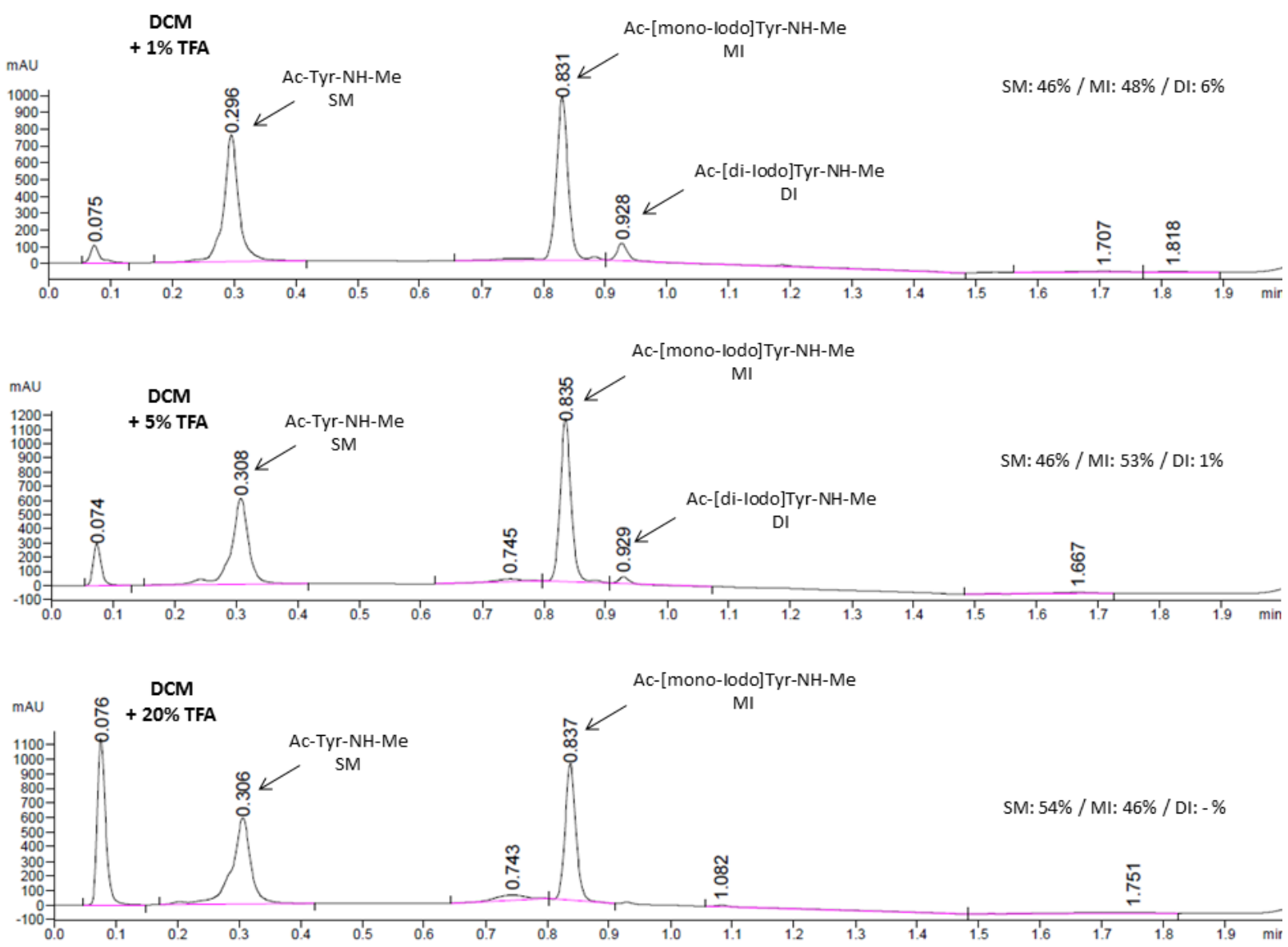


\section{General Mono-iodination procedure}

Tyr-containing peptide was dissolved in DCM+20\% TFA at a concentration of 1-2 mM. Then were added dropwise 1.4 eq of a $50 \mathrm{mM}$ freshly prepared iodination stock solution. Reaction was allowed to stir at room temperature for 15 minutes and was monitored by LC-MS as followed: $10 \mu \mathrm{L}$ of the reaction mixture were quenched with $20 \mu \mathrm{L}$ of distilled water which were submitted to analysis. Depending on the conversion, extra 0.25 eq of iodination stock solution were added sequentially to reach the described ratio of starting material (SM), mono-iodinated product (MI) and di-iodinated product (DI) in Scheme 2. Relative amounts of SM, MI and DI were quantified using AUC integration (absorbance at $215 \mathrm{~nm}$ ) corresponding to their respective masses. Analytical LC-MS chromatograms are shown below:

\section{Cyclo(RGD[mono-iodo]yK)}
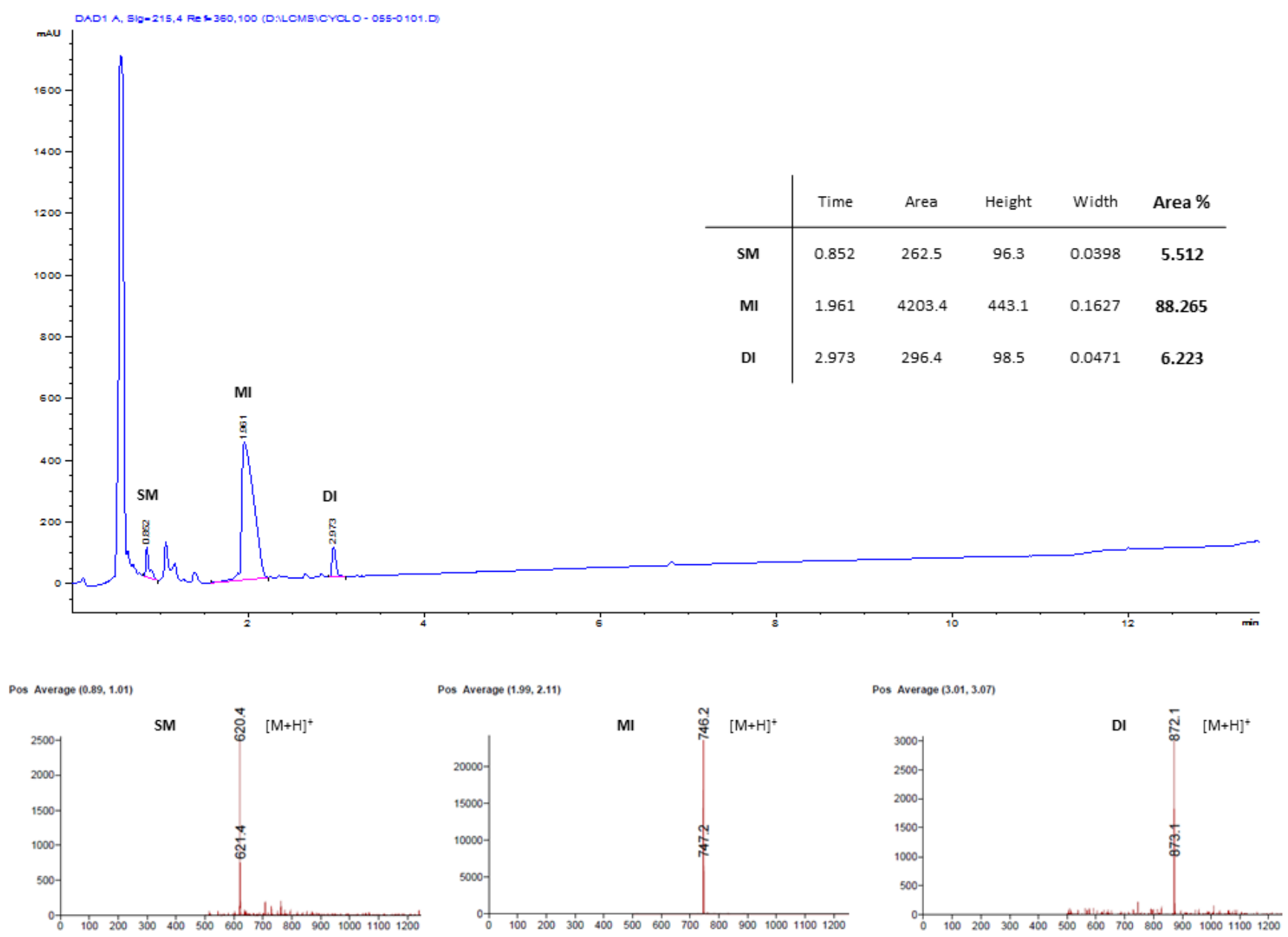


\section{Leucin-Enkephalinamide}

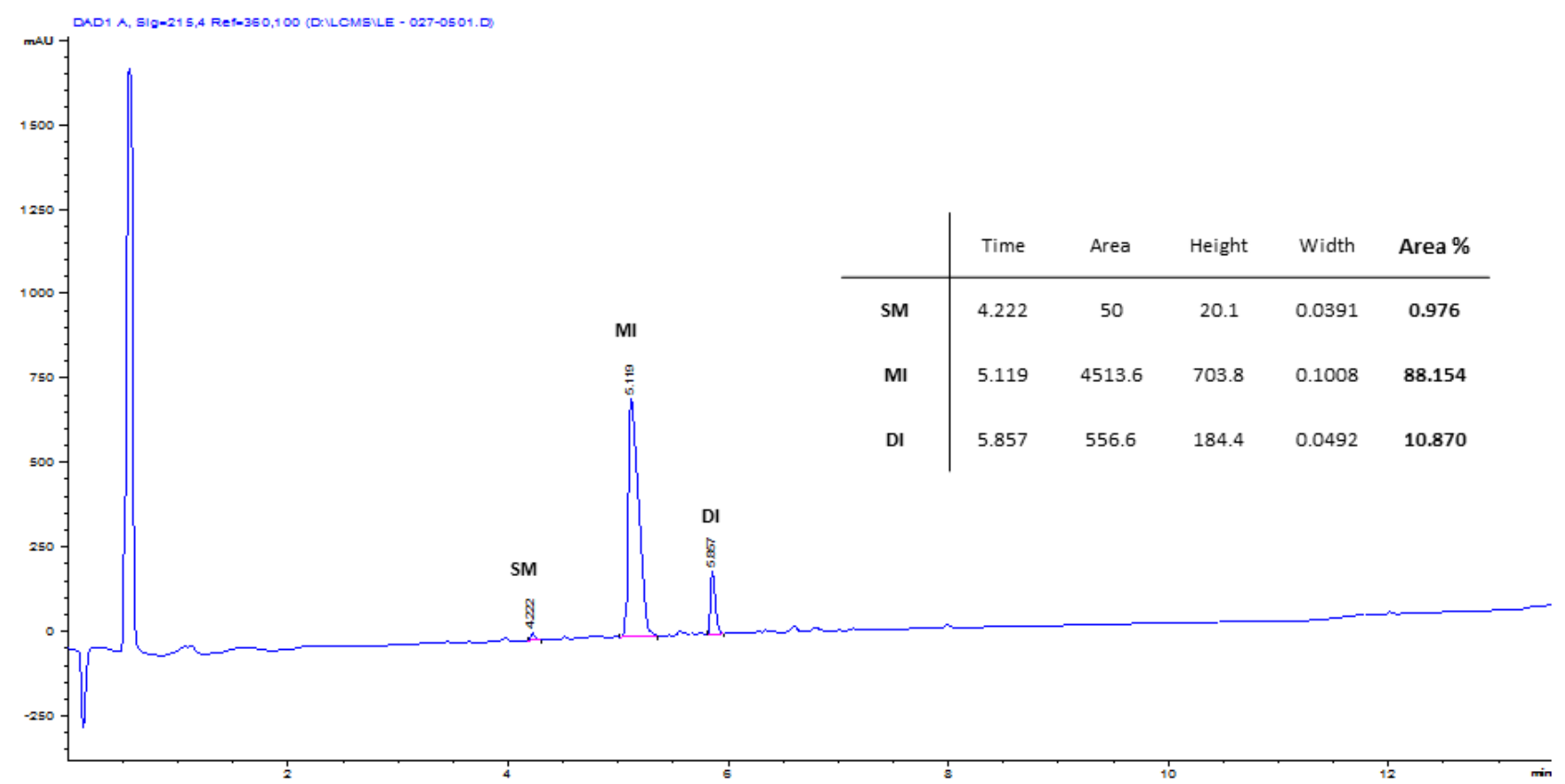

Pos Average $(4,25,4,36)$

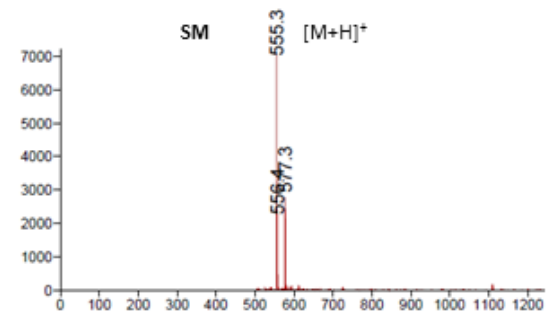

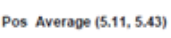

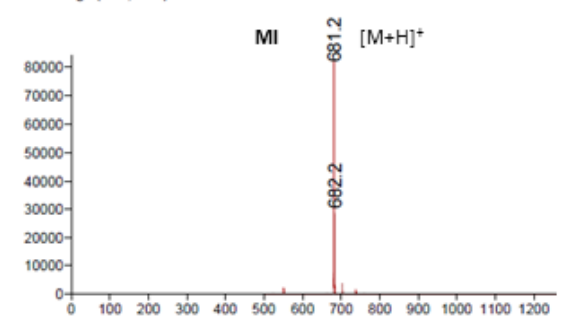

os Average $(5.88,6.04)$

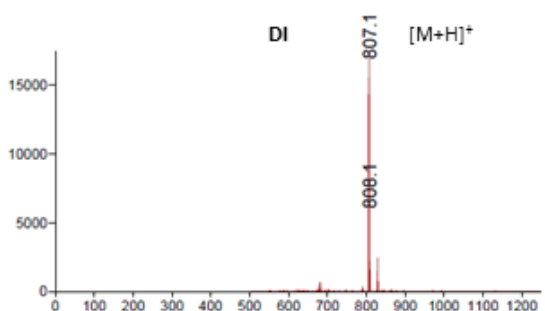




\section{Goserelin}

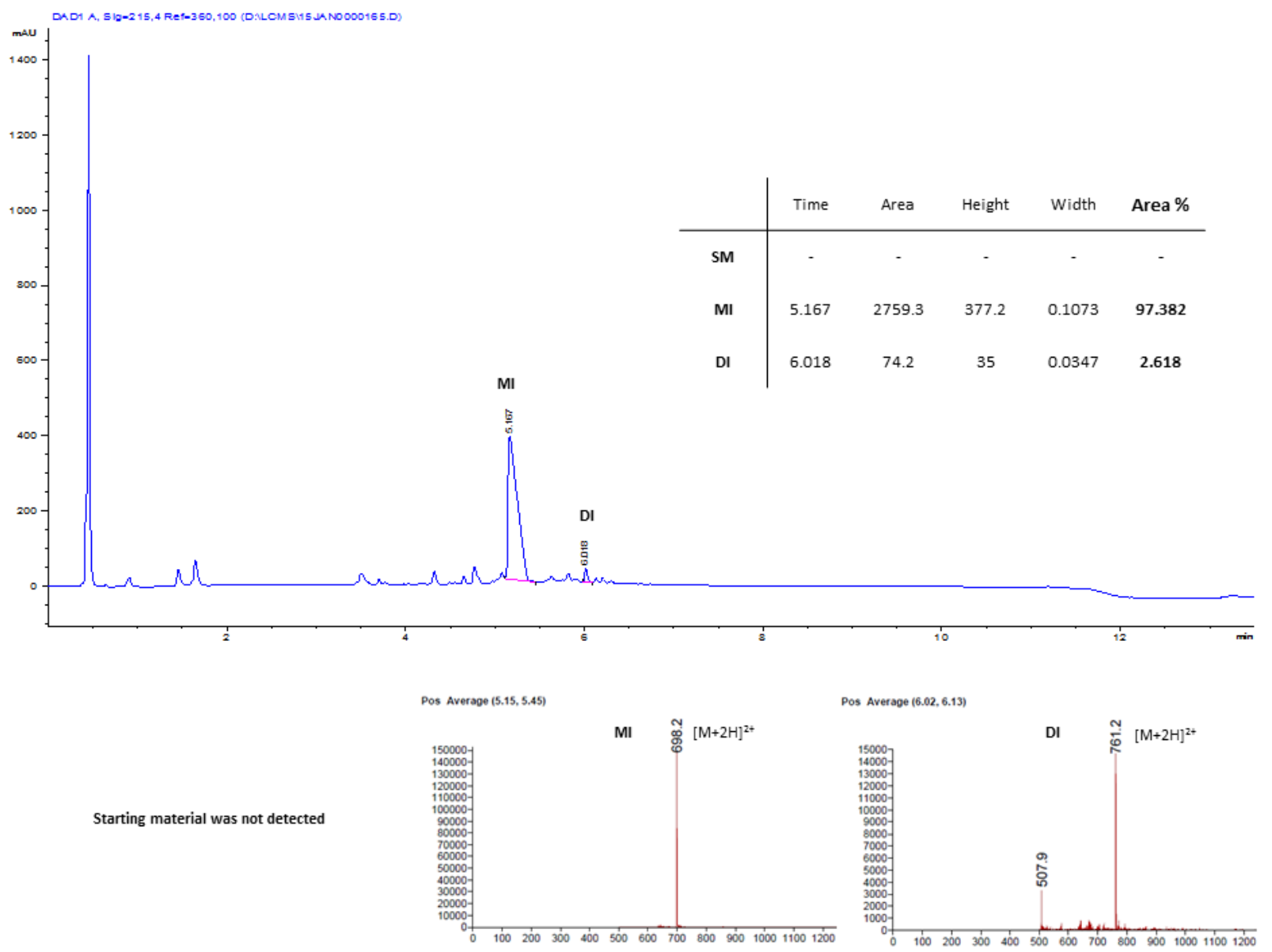




\section{$\left[\mathrm{Tyr}^{0}\right]$-Bradykinin}
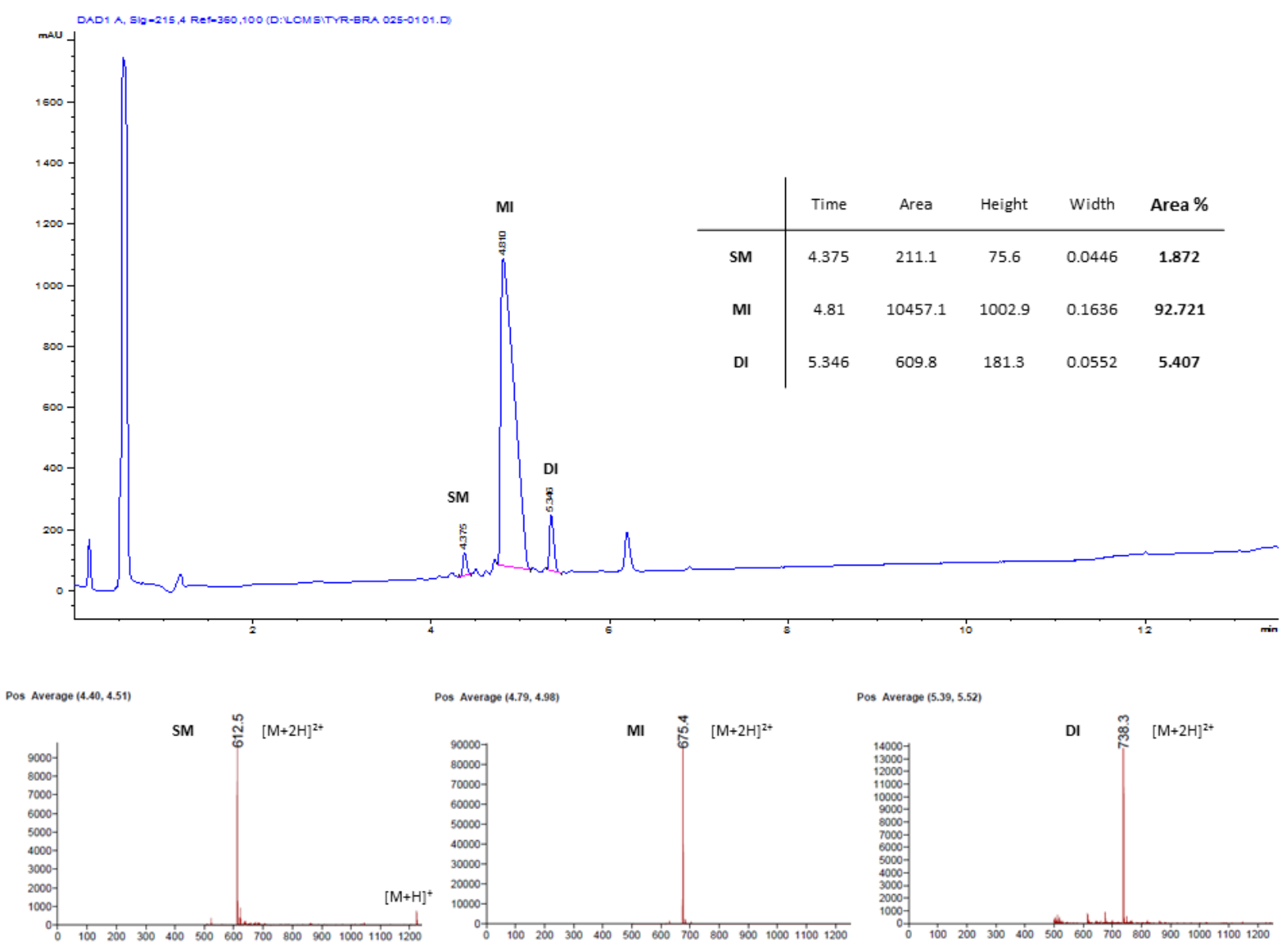


\section{Angiotensin III}
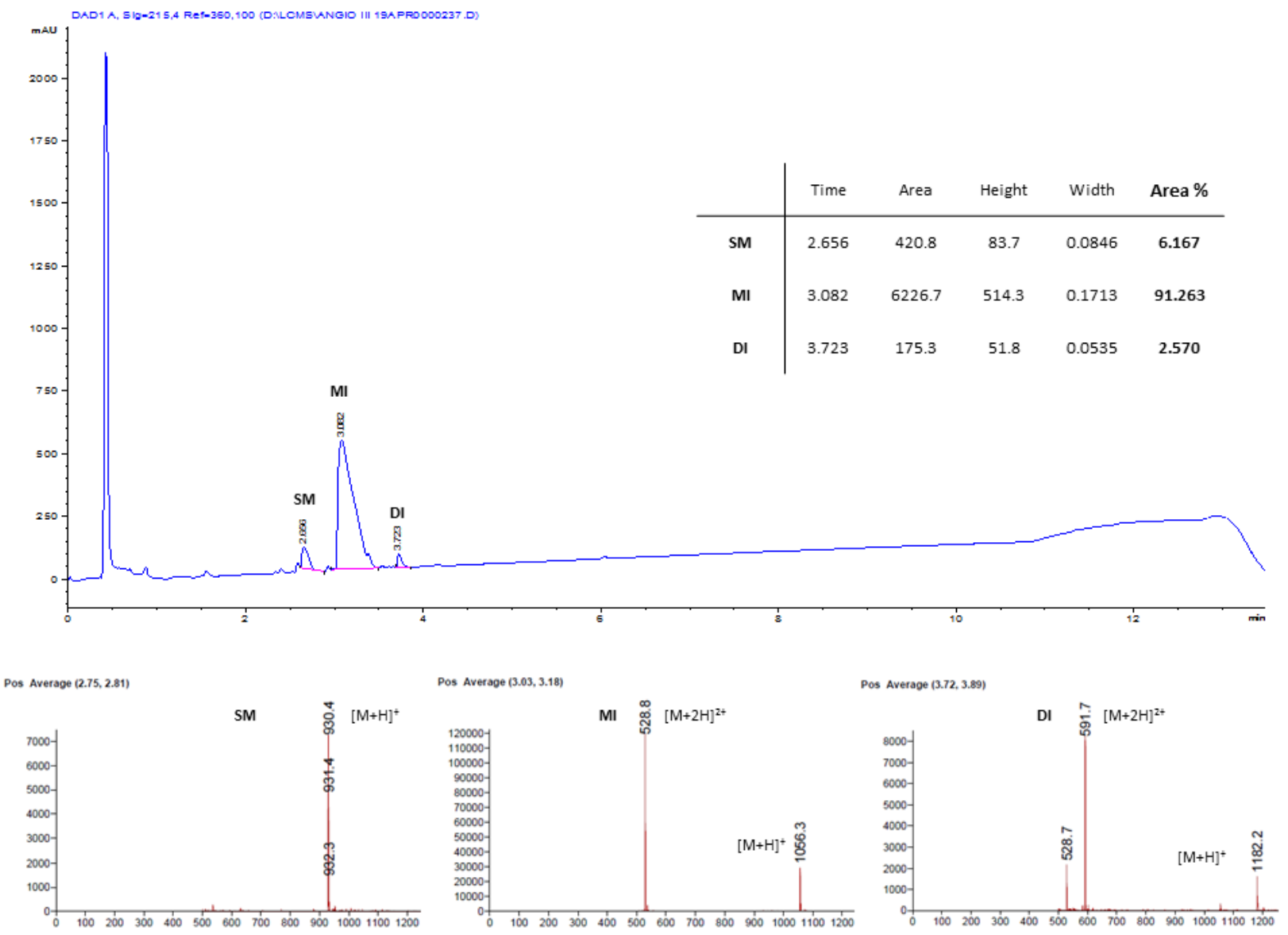


\section{ACP fragment (65-74)}
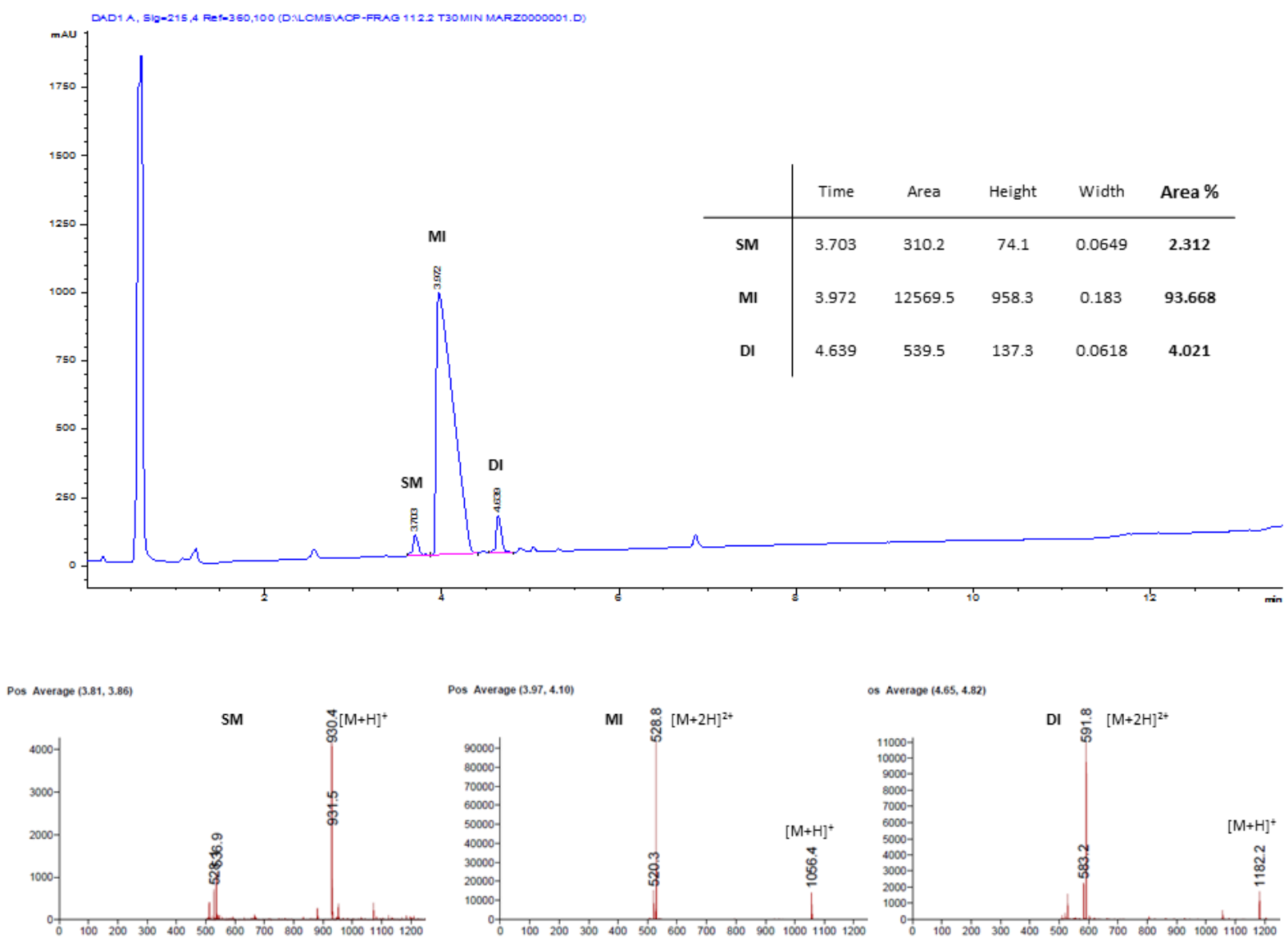


\section{AcMeYVAD-CHO}

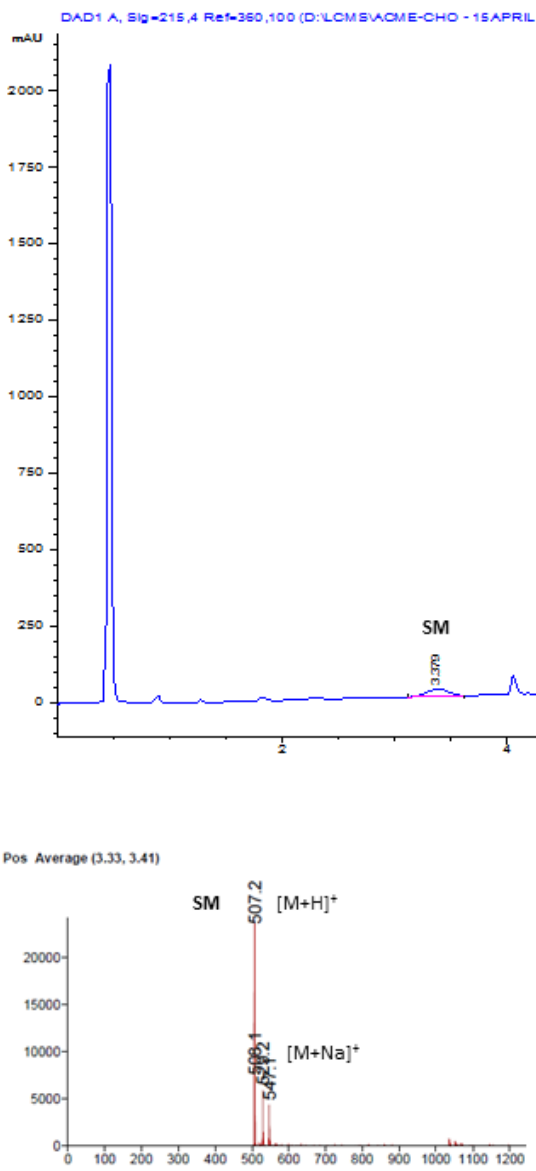

\begin{tabular}{c|ccccc} 
& Time & Area & Height & Width & Area \% \\
\hline SM & 3.379 & 320.1 & 23.9 & 0.204 & $\mathbf{8 . 3 1 2}$ \\
MI & 4.99 & 3531.1 & 257.4 & 0.214 & 91.688 \\
DI & - & - & - & - & -
\end{tabular}

MI

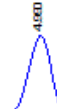

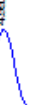

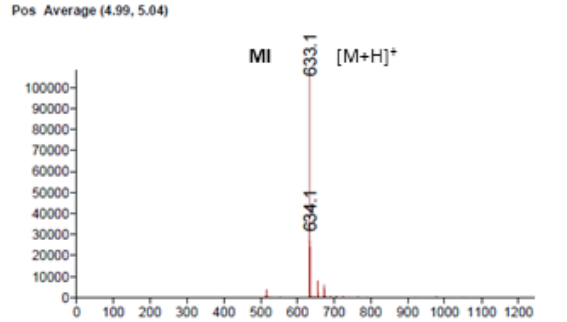

Di-iodinated product was not detected 


\section{Tocinoic acid}
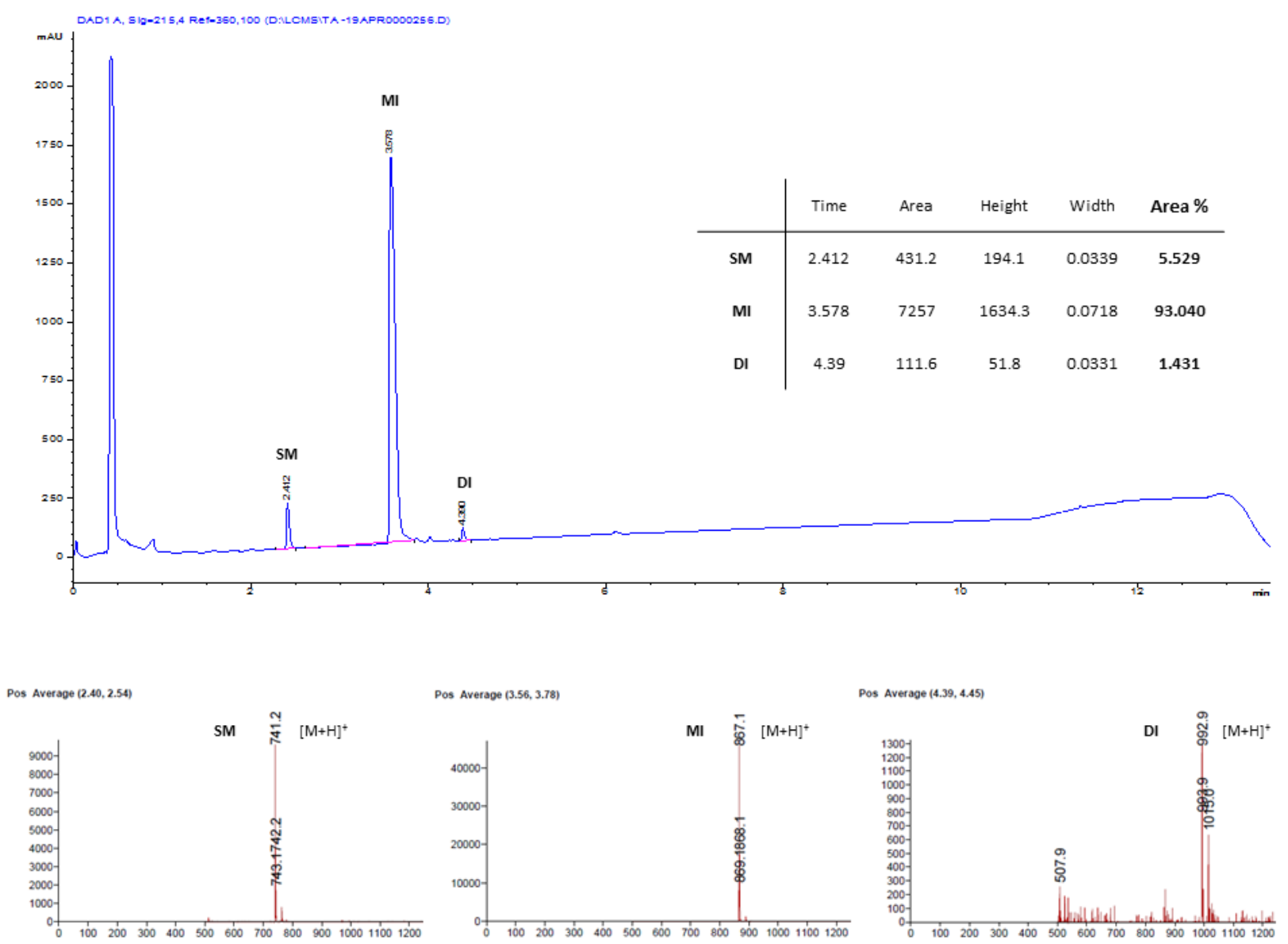


\section{$\left[\right.$ Tyr $\left.^{8}\right]$-Substance P}
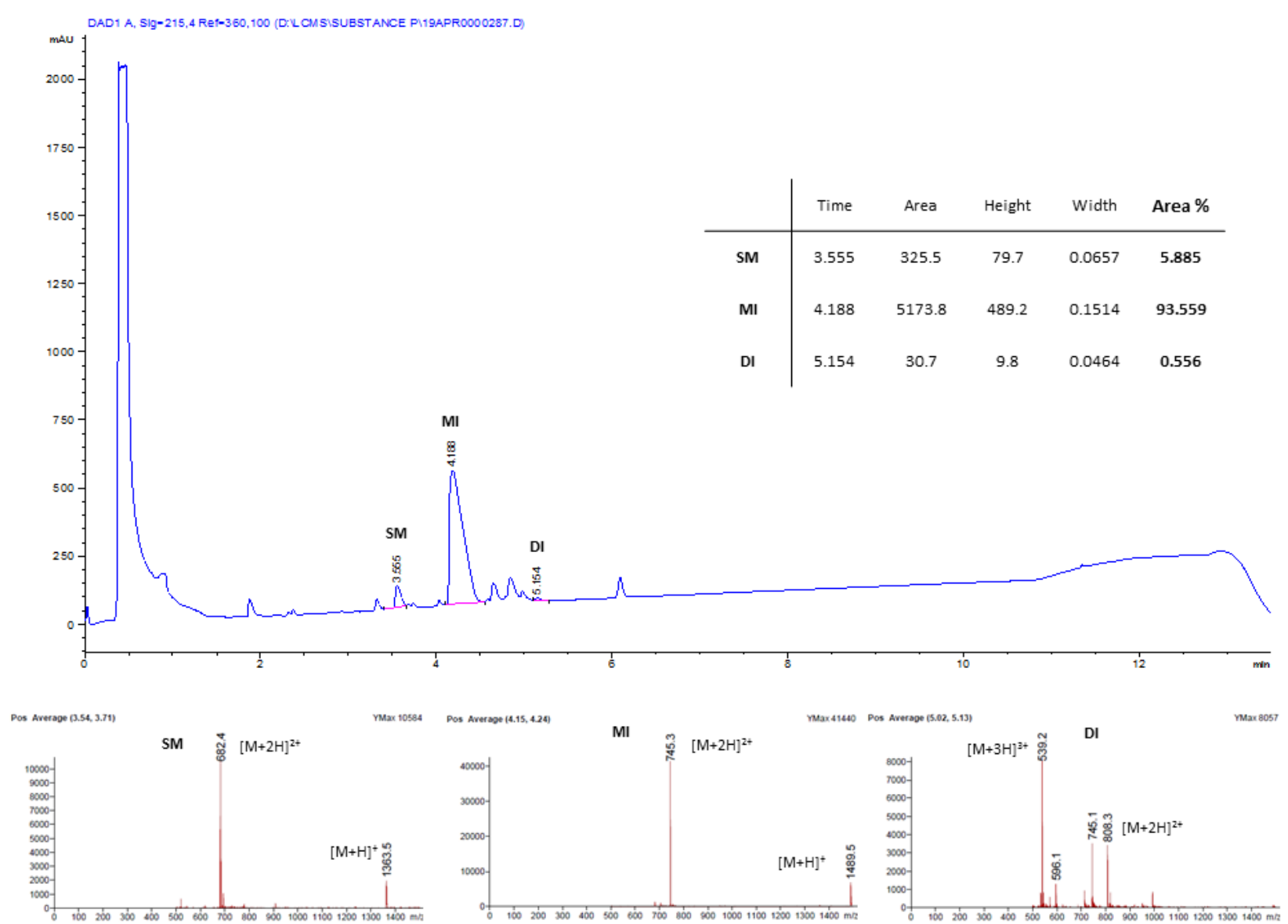


\section{Human GLP-1 (7-37)}
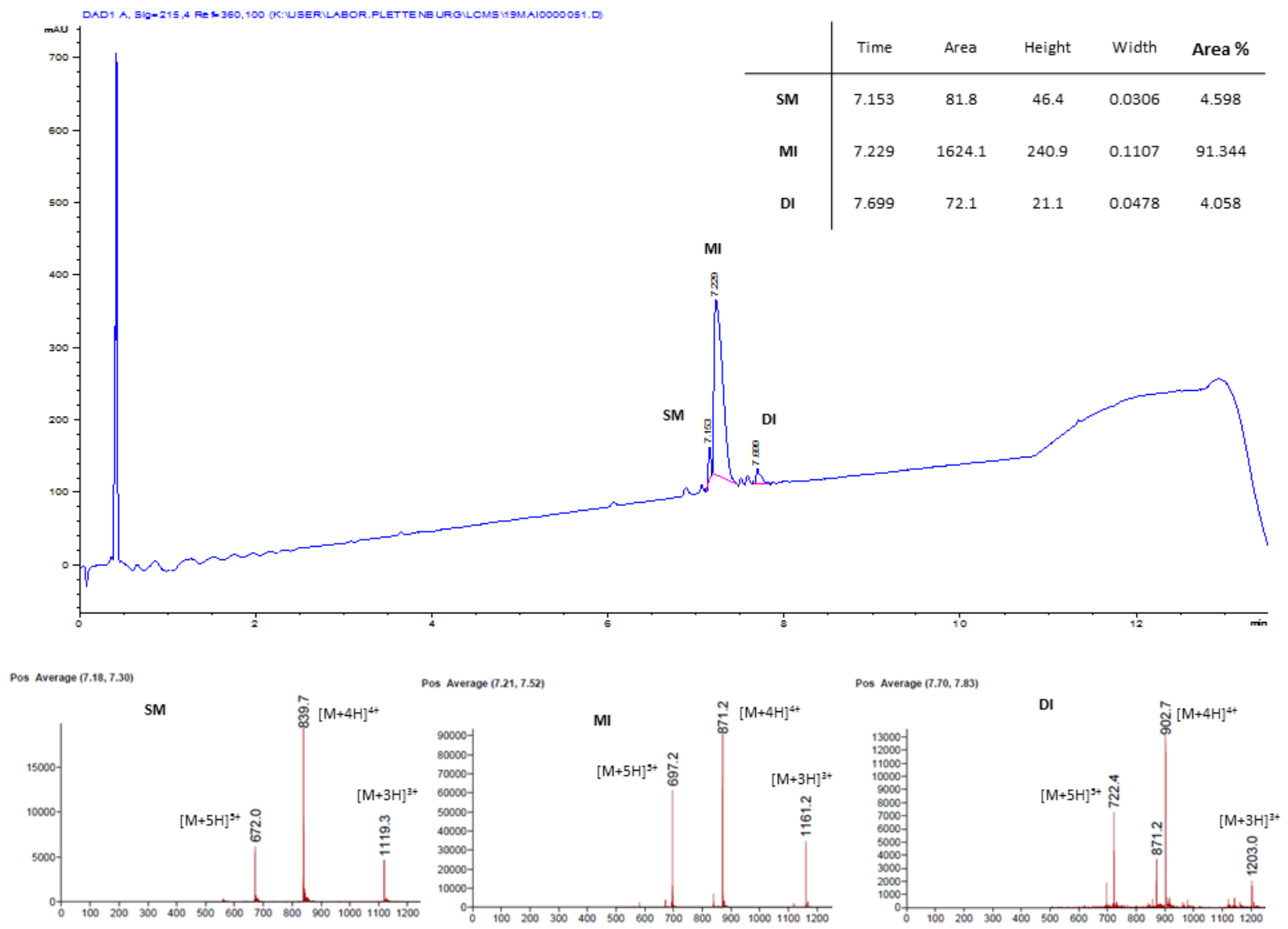


\section{Mono-iodination of Cyclo(RGDyK)}

Cyclo(RGDyK) $(1.02 \mathrm{mg}, 1.18 \mu \mathrm{mol})$ was dissolved in $500 \mu \mathrm{L}$ of DCM and $125 \mu \mathrm{L}$ of TFA. Then were added dropwise $33 \mu \mathrm{L}$ of a $50 \mathrm{mM}$ iodination stock solution ( $1.65 \mu \mathrm{mol}, 1.4$ eq.) (stock solution freshly

prepared: $29.1 \mathrm{mg}$ of Selectfluor were dissolved in $1.6 \mathrm{~mL}$ of ACN before the addition of $12.5 \mathrm{mg}$ of Nal). The reaction mixture was allowed to stir at room temperature for 15 minutes. Two other additions of iodination solution ( $2 \times 5 \mu \mathrm{L}, 2 \times 0.25$ eq.) were needed to reach the following final ratio: SM: $5.5 \%-\mathrm{MI}$ : $88.3 \%$ - DI: $6.2 \%$. The reaction mixture was then evaporated under reduced pressure (TFA was coevaporated three times with DCM) and redissolved in water/acetonitrile (1/1) before being submitted to HPLC (purification performed on Agilent Zorbax Rx C18, gradient: see general methods). The collected fraction was lyophilized to afford the desired product Cyclo(RGD[mono-iodo]yK) ( $\mathrm{m}=0.88 \mathrm{mg}, 0.90 \mu \mathrm{mol}$, $77 \%$ yield).

${ }^{1} \mathrm{H}$ NMR $\left(700 \mathrm{MHz}, \mathrm{d}_{6}\right.$-DMSO, $\left.308 \mathrm{~K}\right) \delta: 1.10(\mathrm{p}, 2 \mathrm{H}), 1.36(\mathrm{~m}, 2 \mathrm{H}), 1.45(\mathrm{~m}, 4 \mathrm{H}), 1.57(\mathrm{br}, 1 \mathrm{H}), 1.72(\mathrm{br}$, $1 \mathrm{H}), 2.39(\mathrm{dd}, 1 \mathrm{H}), 2.70(\mathrm{~m}, 4 \mathrm{H}), 2.78(\mathrm{dd}, 1 \mathrm{H}), 3.08(\mathrm{q}, 2 \mathrm{H}), 3.24(\mathrm{dd}, 1 \mathrm{H}), 3.94(\mathrm{~m}, 1 \mathrm{H}), 4.04(\mathrm{dd}, 1 \mathrm{H})$, $4.16(q, 1 H), 4.34(q, 1 H), 4.63(q, 1 H), 6.77(d, 1 H, J=8.2 \mathrm{~Hz}), 6.87(d d, 1 H, J=8.2,2.0 \mathrm{~Hz}), 7.44(d, 1 H, J$ $=2.0 \mathrm{~Hz}), 7.52(\mathrm{t}, 1 \mathrm{H}), 7.61(\mathrm{~d}, 1 \mathrm{H}), 7.68(\mathrm{br}, 3 \mathrm{H}), 8.00(\mathrm{~d}, 1 \mathrm{H}), 8.09(\mathrm{~d}, 1 \mathrm{H}), 8.11(\mathrm{~d}, 1 \mathrm{H}), 8.44(\mathrm{q}, 1 \mathrm{H})$, $10.10(\mathrm{~s}, 1 \mathrm{H}), 12.20(\mathrm{~s}, 1 \mathrm{H})$.

${ }^{13} \mathrm{C}$ NMR (175 MHz, $\mathrm{d}_{6}$-DMSO, $\left.308 \mathrm{~K}\right)$ ) $8: 22.4,25.1,26.4,28.5,30.6,35.0,35.9,38.6,40.3,43.2,48.8$, 51.8, 54.3, 54.5, 84.1, 114.6, 129.1, 130.2, 138.9, 155.0, 156.6, 169.4, 169.9, 170.5, 171.1, 171.5, 171.8. HRMS (ESI-TOF) Calcd for $\mathrm{C}_{27} \mathrm{H}_{40} \mathrm{IN}_{9} \mathrm{O}_{8}$ : 745.2054; Found: 745.2078.

UPLC-MS rt: $3.257 \mathrm{~min}$ (Gradient 2).

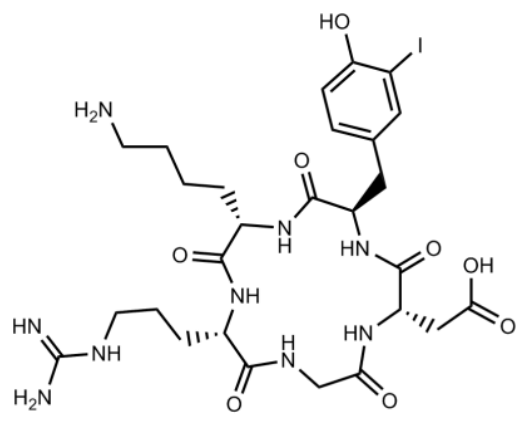



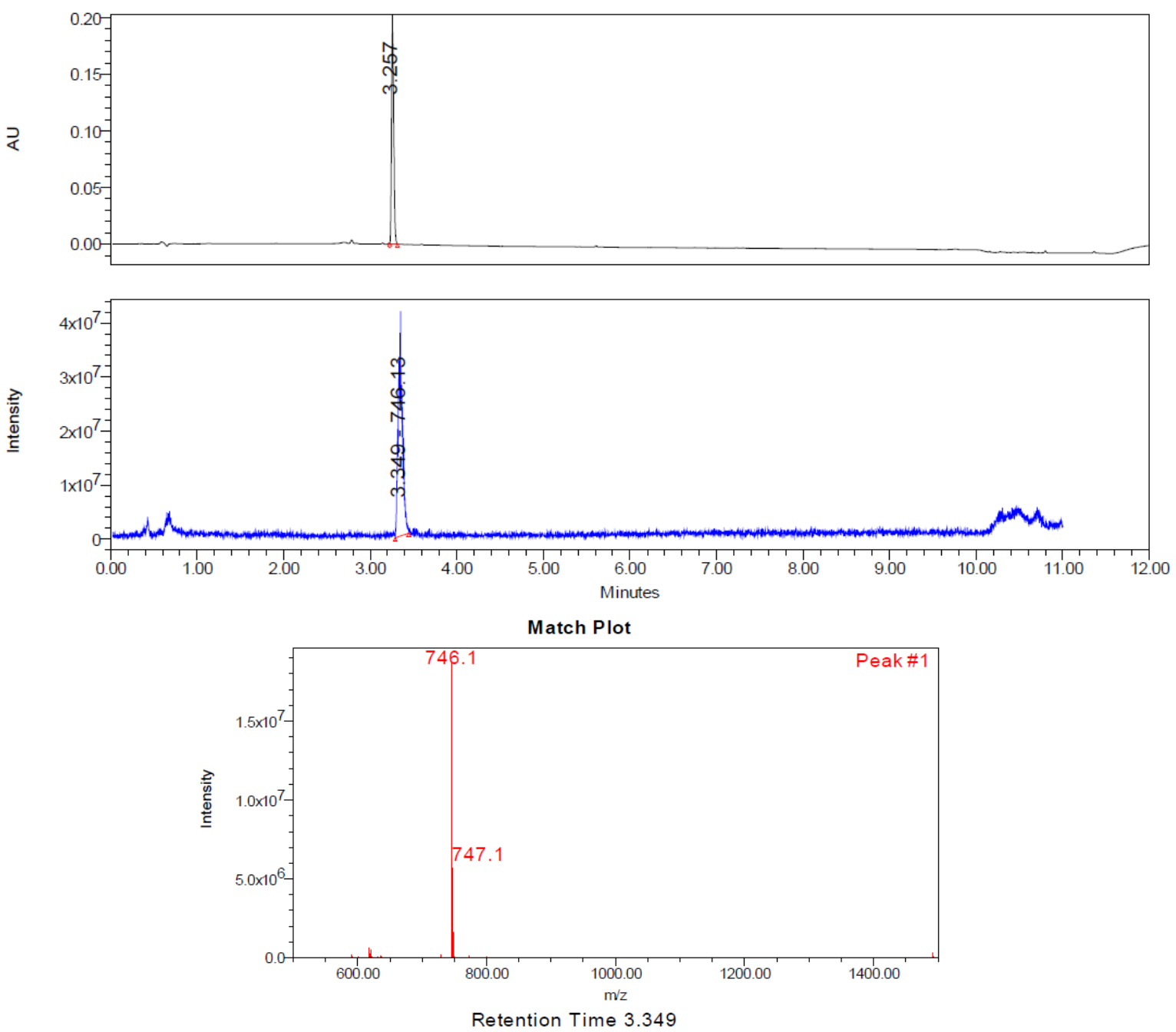


\section{Mono-iodination of Leucin-Enkephalinamide (8)}

Leucin-Enkephalinamide $(36.0 \mathrm{mg}, 64.9 \mu \mathrm{mol})$ was dissolved in $26 \mathrm{~mL}$ of DCM and $6.5 \mathrm{~mL}$ of TFA. Then were added dropwise $1.8 \mathrm{~mL}$ of a $50 \mathrm{mM}$ iodination stock solution ( $91 \mu \mathrm{mol}, 1.4$ eq.) (stock solution freshly prepared: $50.0 \mathrm{mg}$ of Selectfluor were dissolved in $2.8 \mathrm{~mL}$ of ACN before the addition of $21.0 \mathrm{mg}$ of Nal). The reaction mixture was allowed to stir at room temperature for 15 minutes. Two other additions of iodination solution $(2 \times 320 \mu \mathrm{L}, 2 \times 0.25$ eq.) were needed to reach the following final ratio: SM: $1.0 \%$ - MI: $88.2 \%$ - DI: $10.8 \%$. The reaction mixture was then evaporated under reduced pressure (TFA was co-evaporated three times with DCM) and redissolved in water/acetonitrile (1/1) before being submitted to HPLC (purification performed on Xbridge Prep C18, gradient: see general methods). The collected fraction was lyophilized to afford the desired product [mono-lodo]-Leucin-Enkephalinamide (27.3 mg, $40.1 \mu \mathrm{mol}, 62 \%$ yield).

${ }^{1}$ H NMR (500 MHz, d $\mathrm{d}_{6}$-DMSO, $\left.300 \mathrm{~K}\right)$ 8: $0.84(\mathrm{~d}, 3 \mathrm{H}), 0.88(\mathrm{~d}, 3 \mathrm{H}), 1.47(\mathrm{~m}, 2 \mathrm{H}), 1.57(\mathrm{~m}, 1 \mathrm{H}), 2.53(\mathrm{~s}, 1 \mathrm{H})$, $2.80(\mathrm{dd}, 1 \mathrm{H}), 2.87(\mathrm{dd}, 1 \mathrm{H}), 3.02(\mathrm{dd}, 1 \mathrm{H}), 3.45(\mathrm{q}, 1 \mathrm{H}), 3.61(\mathrm{dd}, 1 \mathrm{H}), 3.70(\mathrm{~m}, 3 \mathrm{H}), 4.18(\mathrm{~m}, 1 \mathrm{H}), 4.49(\mathrm{~m}$, $1 \mathrm{H}), 6.77(\mathrm{~d}, 1 \mathrm{H}, \mathrm{J}=8.2 \mathrm{~Hz}), 6.97(\mathrm{~s}, 1 \mathrm{H}), 7.03(\mathrm{dd}, 1 \mathrm{H}, \mathrm{J}=8.2,2.0 \mathrm{~Hz}), 7.17(\mathrm{~m}, 1 \mathrm{H}), 7.25(\mathrm{~d}, 4 \mathrm{H}, \mathrm{J}=4.4$ $\mathrm{Hz}), 7.53(\mathrm{~d}, 1 \mathrm{H}, \mathrm{J}=2.0 \mathrm{~Hz}), 7.96(\mathrm{~d}, 1 \mathrm{H}, \mathrm{J}=8.2 \mathrm{~Hz}), 8.07(\mathrm{~d}, 1 \mathrm{H}, \mathrm{J}=8.2 \mathrm{~Hz}), 8.11(\mathrm{t}, 1 \mathrm{H}, \mathrm{J}=5.6 \mathrm{~Hz}), 8.18(\mathrm{~s}$, $1 \mathrm{H}), 8.30(\mathrm{~s}, 1 \mathrm{H}), 10.10(\mathrm{br}, 1 \mathrm{H})$.

${ }^{13} \mathrm{C}$ NMR (125 MHz, d $\mathrm{d}_{6}$-DMSO, $\left.300 \mathrm{~K}\right) \delta: 21.56,23.02,24.19,37.28,38.53,40.80,41.90,42.02,50.99$, $54.09,55.77,84.36,114.64,126.26,128.04,129.17,130.40,130.62,137.78,139.22,155.08,163.38$, $168.75,169.10,170.69,173.76,173.88$.

HRMS (ESI-TOF) Calcd for $\mathrm{C}_{28} \mathrm{H}_{38} \mathrm{IN}_{6} \mathrm{O}_{6}$ : 681.1892; Found: 681.1891.

UPLC-MS rt: 3.878 min (Gradient 1).
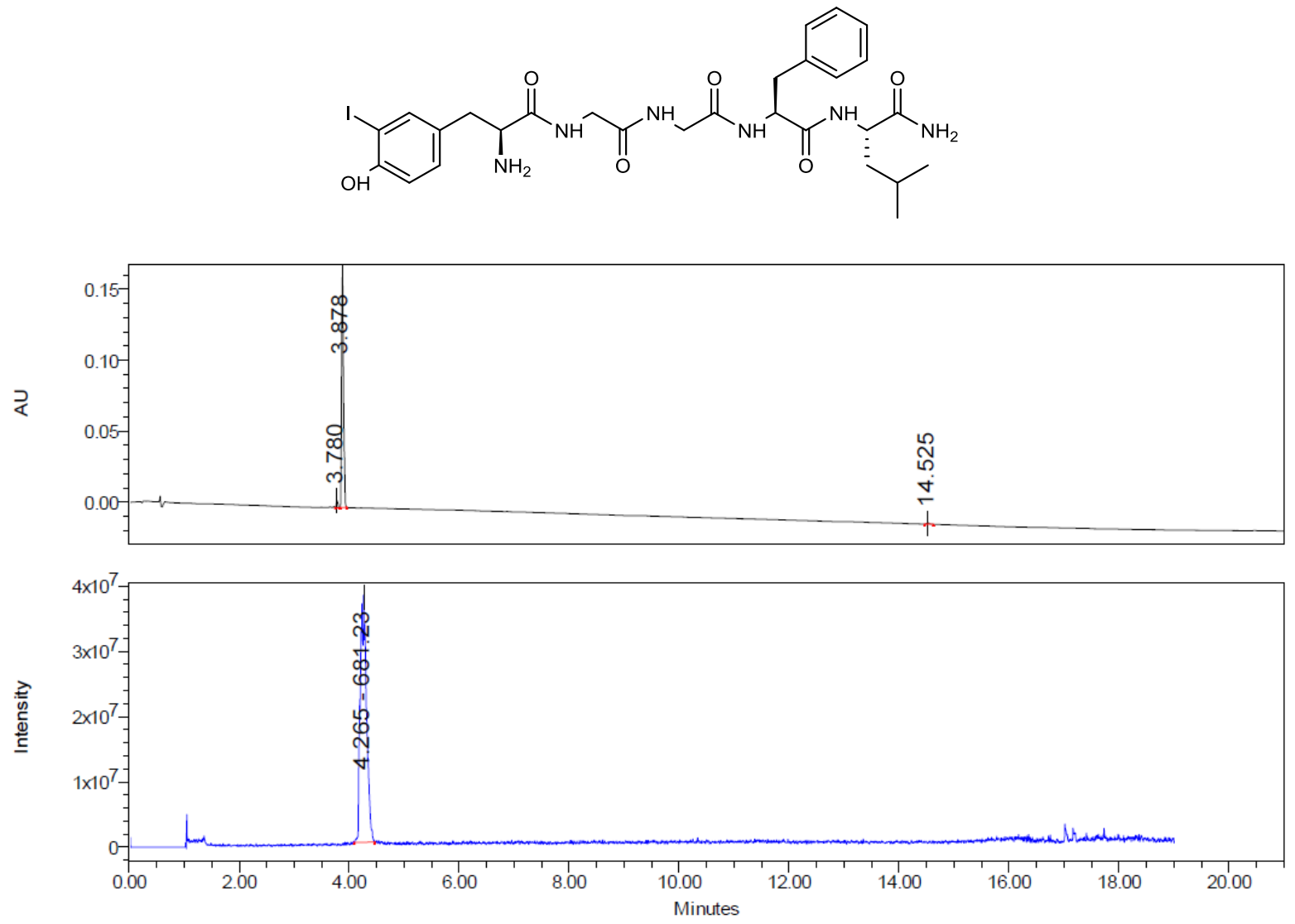


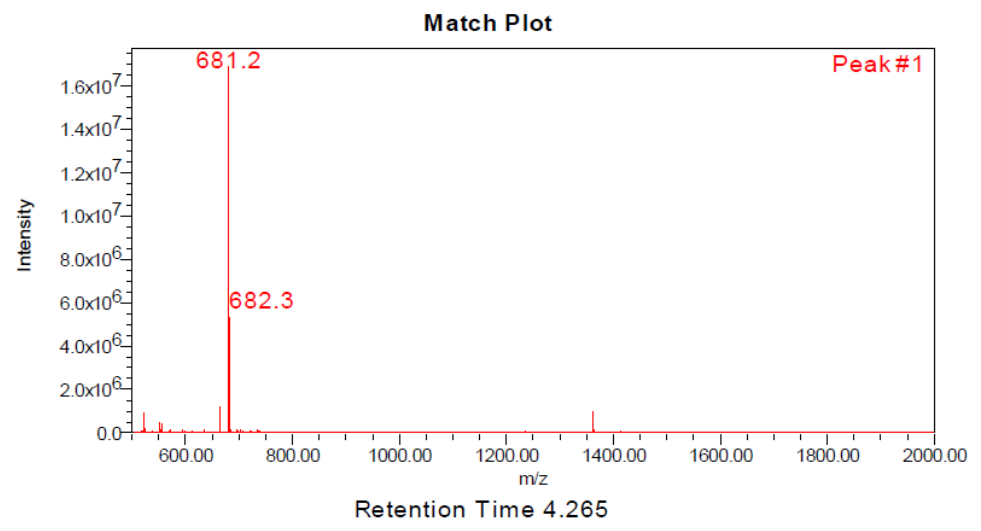

\section{Mono-iodination of [ $\left.\mathrm{Tyr}^{\mathrm{O}}\right]$-Bradykinin}

[Tyr ${ }^{0}$ ]-Bradykinin $(1.58 \mathrm{mg}, 1.29 \mu \mathrm{mol})$ was dissolved in $740 \mu \mathrm{L}$ of DCM and $180 \mu \mathrm{L}$ of TFA. Then were added dropwise $36 \mu \mathrm{L}$ of a $50 \mathrm{mM}$ iodination stock solution (1.80 $\mu \mathrm{mol}, 1.4$ eq.) (stock solution freshly prepared: $29.1 \mathrm{mg}$ of Selectfluor were dissolved in $1.6 \mathrm{~mL}$ of ACN before the addition of $12.5 \mathrm{mg}$ of Nal). The reaction mixture was allowed to stir at room temperature for 15 minutes. Two other additions of iodination solution $(2 \times 6.5 \mu \mathrm{L}, 2 \times 0.25$ eq.) were needed to reach the following final ratio: SM: $1.9 \%$ MI: $92.7 \%$ - DI: $5.4 \%$. The reaction mixture was then evaporated under reduced pressure (TFA was coevaporated three times with DCM) and redissolved in water/acetonitrile (1/1) before being submitted to HPLC (purification performed on Agilent Zorbax Rx C18, gradient: see general methods). The collected fraction was lyophilized to afford the desired product [mono-lodo-Tyr ${ }^{0}$ ]-Bradykinin $(1.46 \mathrm{mg}, 0.93 \mu \mathrm{mol}$, $72 \%$ yield).

${ }^{1}$ H NMR (500 MHz, d $\mathrm{d}_{6}$-DMSO, $\left.300 \mathrm{~K}\right) \delta: 1.43(\mathrm{~m}, 1 \mathrm{H}), 1.51(\mathrm{~m}, 5 \mathrm{H}), 1.61(\mathrm{~m}, 2 \mathrm{H}), 1.70(\mathrm{~m}, 2 \mathrm{H}), 1.79-2.05$ (br, 9H), $2.18(\mathrm{~m}, 1 \mathrm{H}), 2.72(\mathrm{~m}, 2 \mathrm{H}), 2.79(\mathrm{dd}, 1 \mathrm{H}), 2.89(\mathrm{dd}, 1 \mathrm{H}), 2.96(\mathrm{dd}, 1 \mathrm{H}), 3.11(\mathrm{~m}, 5 \mathrm{H}), 3.51(\mathrm{~m}, 2 \mathrm{H})$, $3.62(\mathrm{~m}, 8 \mathrm{H}), 3.99(\mathrm{~m}, 1 \mathrm{H}), 4.20(\mathrm{~m}, 1 \mathrm{H}), 4.26(\mathrm{dd}, 1 \mathrm{H}), 4.30(\mathrm{dd}, 1 \mathrm{H}), 4.49(\mathrm{~m}, 1 \mathrm{H}), 4.50-4.62(\mathrm{~m}, 4 \mathrm{H}), 5.36$ $(\mathrm{s}, 1 \mathrm{H}), 6.78(\mathrm{~d}, 1 \mathrm{H}, \mathrm{J}=8.2 \mathrm{~Hz}), 7.00(\mathrm{dd}, 1 \mathrm{H}, \mathrm{J}=8.2,2.0 \mathrm{~Hz}), 7.15-7.30(\mathrm{~m}, 10 \mathrm{H}), 7.51(\mathrm{~d}, 1 \mathrm{H}, \mathrm{J}=2.0 \mathrm{~Hz})$, $7.56(t, 1 \mathrm{H}), 7.61(\mathrm{t}, 1 \mathrm{H}), 7.70(\mathrm{~d}, 1 \mathrm{H}), 7.94(\mathrm{~m}, 2 \mathrm{H}), 8.08(\mathrm{br}, 2 \mathrm{H}), 8.13(\mathrm{~d}, 1 \mathrm{H}), 8.31(\mathrm{~d}, 1 \mathrm{H}), 8.66(\mathrm{~d}, 1 \mathrm{H})$, $10.31(\mathrm{~s}, 1 \mathrm{H}), 12.72(\mathrm{~s}, 1 \mathrm{H})$.

${ }^{13} \mathrm{C}$ NMR $\left(125 \mathrm{MHz}, \mathrm{d}_{6}\right.$-DMSO, $\left.300 \mathrm{~K}\right) \delta: 23.72,24.43,24.52,25.09,27.90,28.12,28.58,28.81,29.08$, $35.47,37.26,37.72,40.27,40.47,41.67,46.82$, 46.90, 50.01, 51.49, 52.60, 53.12, 53.38, 53.45, 57.63, $59.44,59.60,61.73,84.78,114.66,126.26,126.29,126.90,127.97,128.02,129.10,129.13,130.71$, $137.57,137.68,139.39,155.85,156.66,156.74,167.52,168.48,168.50,169.63,170.04,170.85,170.95$, $170.96,171.77,173.08$.

HRMS (ESI-TOF) Calcd for $\mathrm{C}_{59} \mathrm{H}_{81} \mathrm{IN}_{16} \mathrm{O}_{13}$ : 1348.5214; Found: 1348.5255.

UPLC-MS rt: 2.664 min (Gradient 1). 

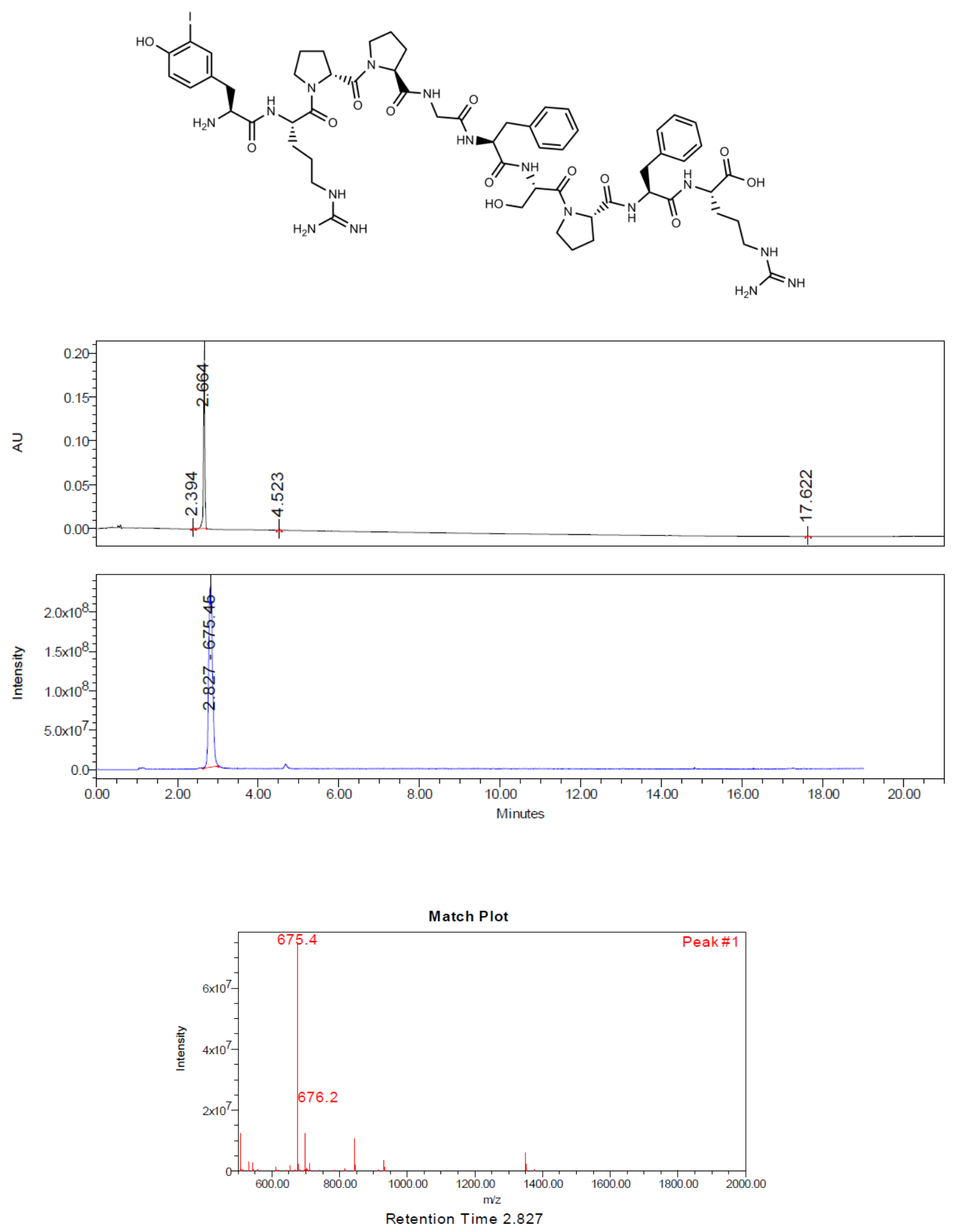


\section{Mono-iodination of Angiotensin III}

Angiotensin III $(20.0 \mathrm{mg}, 15.7 \mu \mathrm{mol})$ was dissolved in $8.5 \mathrm{~mL}$ of DCM and $2.0 \mathrm{~mL}$ of TFA. Then were added dropwise $440 \mu \mathrm{L}$ of a $50 \mathrm{mM}$ iodination stock solution ( $22.0 \mu \mathrm{mol}, 1.4$ eq.) (stock solution freshly prepared: $14.8 \mathrm{mg}$ of Selectfluor were dissolved in $830 \mu \mathrm{L}$ of ACN before the addition of $6.9 \mathrm{mg}$ of Nal). The reaction mixture was allowed to stir at room temperature for 15 minutes. Three other additions of iodination solution ( $3 \times 80 \mu \mathrm{L}, 3 \times 0.25$ eq.) were needed to reach the following final ratio: SM: $6.2 \%-\mathrm{MI}$ : 91.3\% - DI: $2.5 \%$. The reaction mixture was then evaporated under reduced pressure (TFA was coevaporated three times with DCM) and redissolved in water/acetonitrile (1/1) before being submitted to HPLC (purification performed on Xbridge Prep C18, gradient: see general methods). The collected fraction was lyophilized to afford the desired product [mono-lodo]-Angiotensin III (14.9 mg, $11.6 \mu \mathrm{mol}$, $74 \%$ yield).

${ }^{1}$ H NMR (500 MHz, d N $\left.^{-D M S O}, 300 \mathrm{~K}\right) \delta: 0.75(\mathrm{~d}, 3 \mathrm{H}), 0.77(\mathrm{t}, 3 \mathrm{H}), 0.79(\mathrm{~d}, 3 \mathrm{H}), 0.84(\mathrm{~d}, 3 \mathrm{H}), 1.05(\mathrm{p}, 1 \mathrm{H})$, $1.36(\mathrm{~m}, 1 \mathrm{H}), 1.46(\mathrm{~m}, 2 \mathrm{H}), 1.64(\mathrm{~m}, 4 \mathrm{H}), 1.77(\mathrm{br}, 2 \mathrm{H}), 1.99(\mathrm{~m}, 2 \mathrm{H}), 2.59(\mathrm{dd}, 1 \mathrm{H}), 2.77(\mathrm{dd}, 1 \mathrm{H}), 2.85(\mathrm{dd}$, 1H), $2.93(\mathrm{dd}, 1 \mathrm{H}), 3.07(\mathrm{~m}, 4 \mathrm{H}), 3.46(\mathrm{br}, 1 \mathrm{H}), 3.61(\mathrm{~m}, 1 \mathrm{H}), 3.87(\mathrm{br}, 1 \mathrm{H}), 4.15(\mathrm{t}, 1 \mathrm{H}), 4.27(\mathrm{~m}, 2 \mathrm{H}), 4.38$ (dd, 1H), $4.49(\mathrm{~m}, 1 \mathrm{H}), 4.79(\mathrm{br}, 1 \mathrm{H}), 6.75(\mathrm{~d}, \mathrm{~J}=8.4 \mathrm{~Hz}, 1 \mathrm{H}), 7.08(\mathrm{~m}, 2 \mathrm{H}), 7.17(\mathrm{~m}, 1 \mathrm{H}), 7.23(\mathrm{~m}, 5 \mathrm{H}), 7.38$ (br, 1H), $7.60(\mathrm{~m}, 2 \mathrm{H}), 7.99(\mathrm{~s}, 1 \mathrm{H}), 8.01(\mathrm{~s}, 1 \mathrm{H}), 8.10(\mathrm{br}, 4 \mathrm{H}), 8.35(\mathrm{~d}, 2 \mathrm{H}), 8.41(\mathrm{~d}, 2 \mathrm{H}), 8.93(\mathrm{br}, 1 \mathrm{H})$, $10.17(\mathrm{~s}, 1 \mathrm{H}), 14.19$ (br, 1H).

${ }^{13} \mathrm{C}$ NMR $\left(125 \mathrm{MHz}, \mathrm{d}_{6}\right.$-DMSO, $\left.300 \mathrm{~K}\right) \delta: 10.85,15.19,17.57,19.19,24.10,24.25,24.28,26.30,28.62$, 29.13, 31.11, 35.77, 36.62, 37.01, 40.12, 46.99, 47.7, 51.72, 53.83, 53.93, 56.65, 57.12, 59.87, 84.27, $114.41,117.1,126.2,128.00,129.17,130.29,130.36,133.8,137.88,138.97,155.02,156.71,168.22$, $170.48,170.85,170.97,171.43,172.55$.

HRMS (ESI-TOF) Calcd for $\mathrm{C}_{46} \mathrm{H}_{66} \mathrm{IN}_{13} \mathrm{O}_{8}$ : 1055.4202; Found: 1055.4242.

UPLC-MS rt: $4.481 \mathrm{~min}$ (Gradient 2).

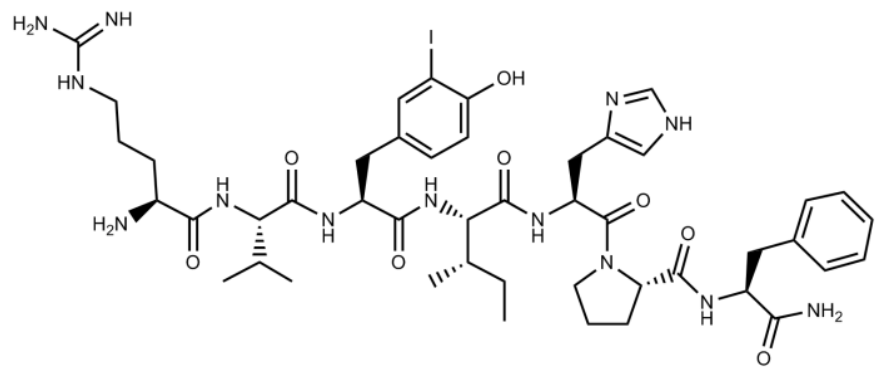



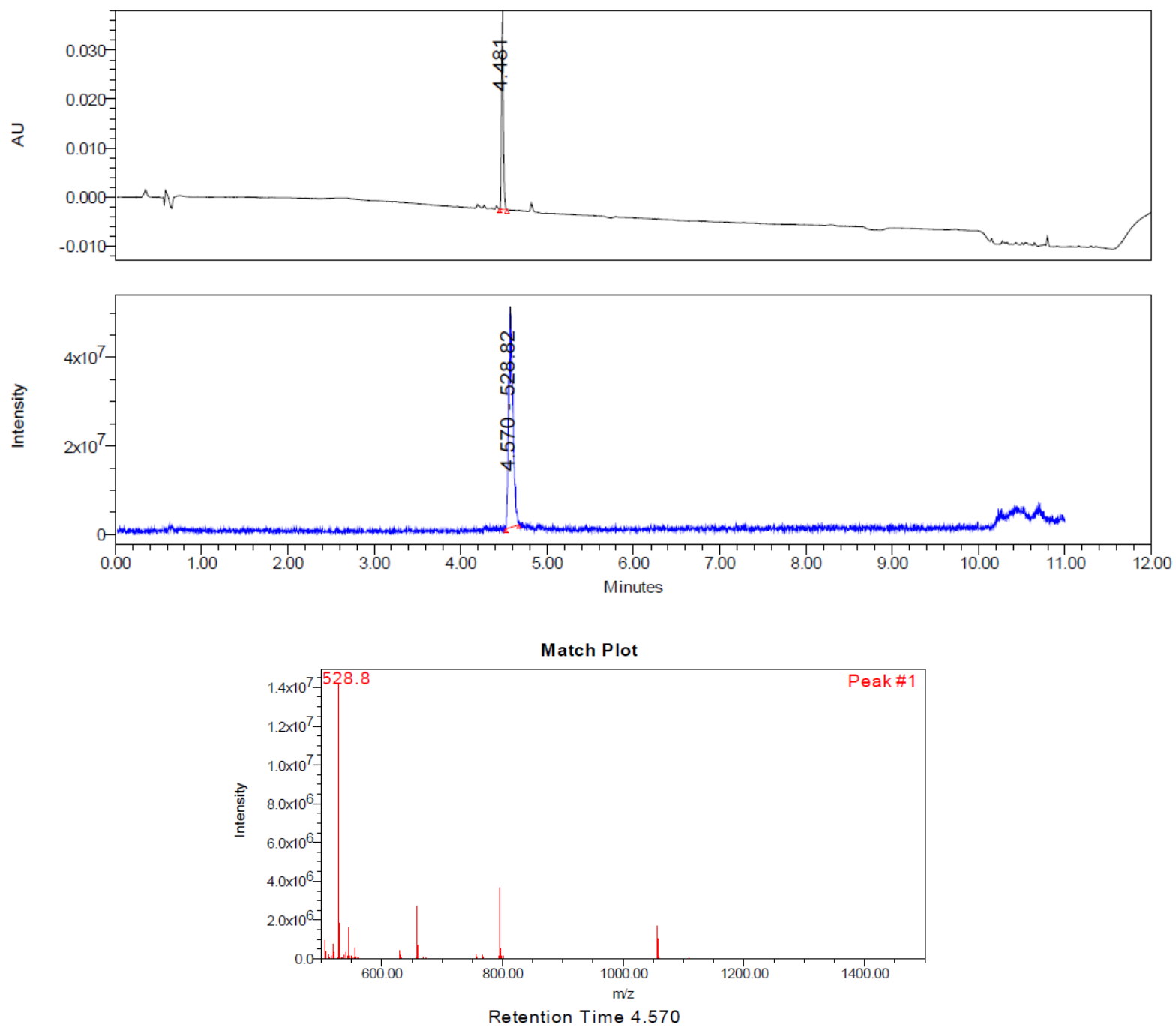


\section{Mono-iodination of ACP-fragment 65-74 (11)}

ACP fragment $65-74(18.0 \mathrm{mg}, 15.3 \mu \mathrm{mol})$ was dissolved in $6.0 \mathrm{~mL}$ of DCM and $1.5 \mathrm{~mL}$ of TFA. Then were added dropwise $428 \mu \mathrm{L}$ of a $50 \mathrm{mM}$ iodination stock solution $(21.4 \mu \mathrm{mol}, 1.4$ eq.) (stock solution freshly prepared: $15.3 \mathrm{mg}$ of Selectfluor were dissolved in $860 \mu \mathrm{L}$ of $\mathrm{ACN}$ before the addition of $7.0 \mathrm{mg}$ of Nal). The reaction mixture was allowed to stir at room temperature for 15 minutes. Two other additions of iodination solution ( $2 \times 45 \mu \mathrm{L}, 2 \times 0.1$ eq.) were needed to reach the following final ratio: SM: $2.3 \%$ - MI: 93.7\% - DI: $4.0 \%$. The reaction mixture was then evaporated under reduced pressure (TFA was coevaporated three times with DCM) and redissolved in water/acetonitrile (1/1) before being submitted to HPLC (purification performed on Xbridge Prep C18, gradient: see general methods). The collected fractions were lyophilized to afford the desired product [mono-lodo]-ACP fragment 65-74 (11.5 mg, 9.7 umol, $63 \%$ yield).

${ }^{1}$ H NMR (500 MHz, d N $\left.^{-D M S O}, 295 \mathrm{~K}\right) \delta: 0.73(\mathrm{~d}, 3 \mathrm{H}), 0.76(\mathrm{t}, 3 \mathrm{H}), 0.80(\mathrm{~m}, 6 \mathrm{H}), 0.91(\mathrm{~d}, 3 \mathrm{H}), 0.92(\mathrm{~d}, 3 \mathrm{H})$, $1.00(\mathrm{~m}, 1 \mathrm{H}), 1.06(\mathrm{~m}, 1 \mathrm{H}), 1.17(\mathrm{~d}, 3 \mathrm{H}), 1.18(\mathrm{~d}, 3 \mathrm{H}), 1.35(\mathrm{~m}, 1 \mathrm{H}), 1.43(\mathrm{~m}, 1 \mathrm{H}), 1.66(\mathrm{~m}, 2 \mathrm{H}), 1.76(\mathrm{~m}$, $1 \mathrm{H}), 1.87(\mathrm{~m}, 1 \mathrm{H}), 2.03(\mathrm{~m}, 1 \mathrm{H}), 2.13(\mathrm{~m}, 2 \mathrm{H}), 2.43(\mathrm{~m}, 1 \mathrm{H}), 2.51(\mathrm{~m}, 1 \mathrm{H}), 2.62(\mathrm{~m}, 3 \mathrm{H}), 2.84(\mathrm{dd}, 1 \mathrm{H}), 3.58$ $(\mathrm{m}, 3 \mathrm{H}), 4.09(\mathrm{t}, 1 \mathrm{H}), 4.12(\mathrm{t}, 1 \mathrm{H}), 4.28(\mathrm{~m}, 1 \mathrm{H}), 4.32(\mathrm{~m}, 2 \mathrm{H}), 4.44(\mathrm{~m}, 2 \mathrm{H}), 4.51(\mathrm{~m}, 1 \mathrm{H}), 6.74(\mathrm{~d}, \mathrm{~J}=8.2$ $\mathrm{Hz}, 1 \mathrm{H}), 6.84(\mathrm{~s}, 1 \mathrm{H}), 6.99(\mathrm{br}, 1 \mathrm{H}), 7.02(\mathrm{dd}, \mathrm{J}=8.2,1.8 \mathrm{~Hz}, 1 \mathrm{H}), 7.14(\mathrm{~s}, 1 \mathrm{H}), 7.19(\mathrm{~s}, 1 \mathrm{H}), 7.28(\mathrm{~s}, 1 \mathrm{H})$, $7.46(\mathrm{~s}, 1 \mathrm{H}), 7.52(\mathrm{~d}, \mathrm{~J}=1.8 \mathrm{~Hz}, 1 \mathrm{H}), 7.67(\mathrm{~d}, 1 \mathrm{H}), 7.77(\mathrm{~d}, 1 \mathrm{H}), 8.04(\mathrm{~m}, 5 \mathrm{H}), 8.10(\mathrm{~d}, 1 \mathrm{H}), 8.17(\mathrm{~m}, 2 \mathrm{H})$, $8.28(\mathrm{~d}, 1 \mathrm{H}), 8.52(\mathrm{~d}, 1 \mathrm{H}), 10.07(\mathrm{~s}, 1 \mathrm{H}), 12.35(\mathrm{~s}, 1 \mathrm{H})$.

${ }^{13} \mathrm{C}$ NMR $\left(125 \mathrm{MHz}, \mathrm{d}_{6}\right.$-DMSO, $\left.295 \mathrm{~K}\right) \delta: 11.08,11.11,15.15,15.27,17.07,17.79,18.22,18.33,24.01$, 24.45, 28.02, 29.89, 31.29, 35.96, 36.27, 36.43, 36.57, 36.99, 42.41, 47.97, 48.02, 49.54, 49.92, 52.07, $53.94,56.6,57.18,57.25,84.22,114.49,130.19,130.34,139.1,155.01,167.63,170.29,170.47,170.6$, $170.83,171.01,171.07,171.11,171.65,171.82,171.86,171.91,173.73$.

HRMS (ESI-TOF) Calcd for $\mathrm{C}_{47} \mathrm{H}_{74} \mathrm{IN}_{13} \mathrm{O}_{15}$ : 1187.4472; Found: 1187.4526.

UPLC-MS rt: 3.774 min (Gradient 1).<smiles>CC[C@H](C)[C@H](NC(=O)[C@H](C)NC(=O)[C@H](C)NC(=O)[C@H](CCC(N)=O)NC(=O)[C@@H](N)C(C)C)C(=O)N[C@@H](CC(=O)O)C(=O)N[C@@H](Cc1ccc(O)c(I)c1)C(=O)N[C@@H](C(=O)N[C@@H](CC(N)=O)C(=O)NCC(N)=O)[C@@H](C)CC</smiles> 

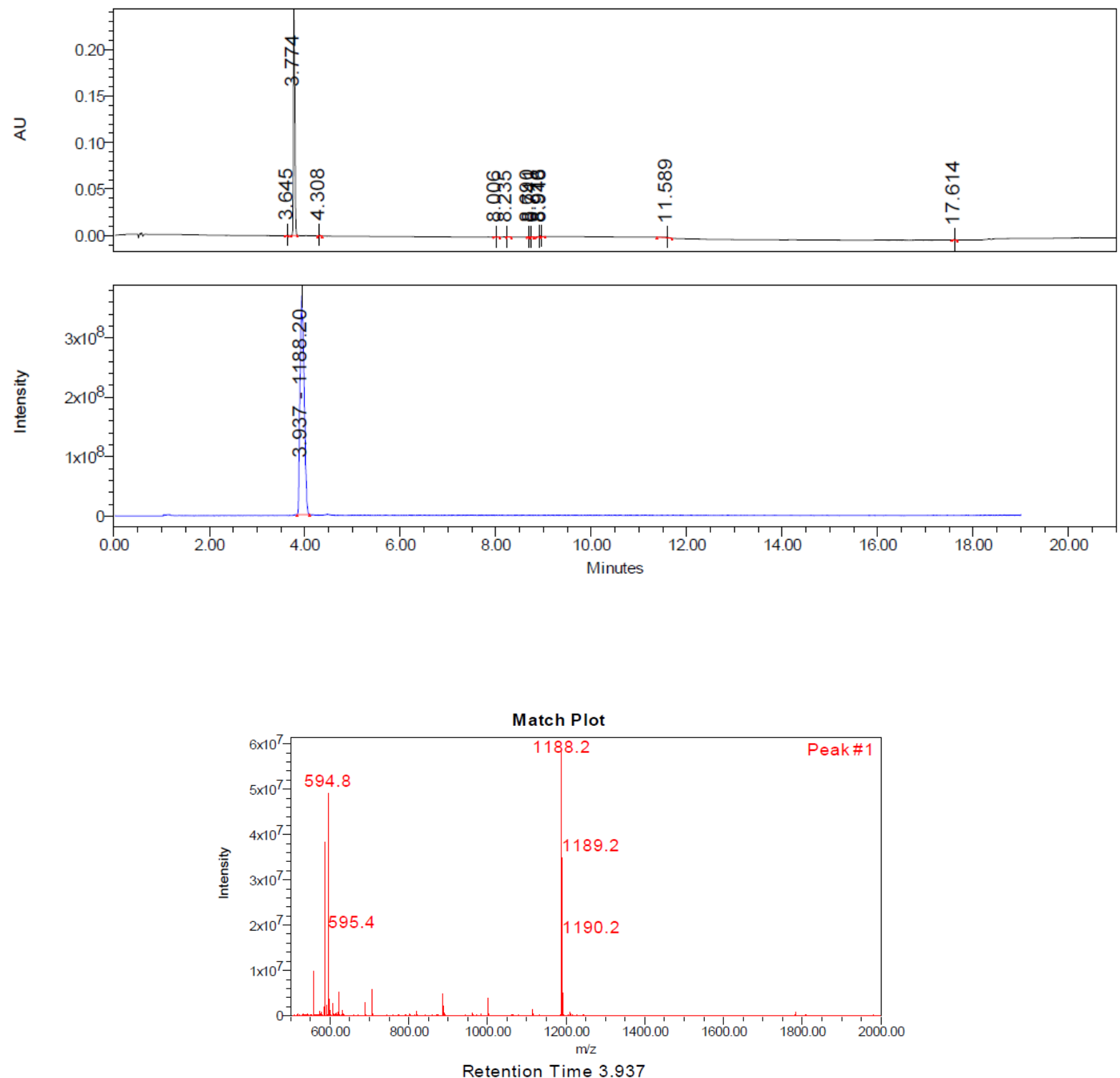


\section{Mono-iodination of $\left[\mathrm{Tyr}^{8}\right]$-Substance $\mathbf{P}(\mathbf{7})$}

$\left[\right.$ Tyr $^{8}$ ]-Substance $P(3.0 \mathrm{mg}, 2.2 \mu \mathrm{mol})$ was dissolved in $1.4 \mathrm{~mL}$ of DCM and $300 \mu \mathrm{L}$ of TFA. Then were added dropwise $60 \mu \mathrm{L}$ of a $50 \mathrm{mM}$ iodination stock solution $(3.0 \mu \mathrm{mol}, 1.4$ eq.) (stock solution freshly prepared: $15.0 \mathrm{mg}$ of Selectfluor were dissolved in $840 \mu \mathrm{L}$ of $\mathrm{ACN}$ before the addition of $7.2 \mathrm{mg}$ of Nal). The reaction mixture was allowed to stir at room temperature for 15 minutes. Three other additions of iodination solution ( $3 \times 15 \mu \mathrm{L}, 3 \times 0.35$ eq.) were needed to reach the following final ratio: SM: $5.9 \%$ - MI: 93.6\% - DI: $0.5 \%$. The reaction mixture was then quenched with $400 \mu \mathrm{L}$ of reducing cocktail (freshly prepared: $\mathrm{KI}(10 \mathrm{mg})$ and ascorbic acid (10 mg) were sonicated in $500 \mu \mathrm{L}$ of TFA for 10 minutes) and evaporated under reduced pressure (TFA was co-evaporated three times with DCM). The crude was dissolved in water/acetonitrile (1/1) before being submitted to HPLC (purification performed on Agilent Zorbax Rx C18, gradient: see general methods). The collected fractions were lyophilized to afford the desired product [mono-lodo-Tyr ${ }^{8}$ ]-Substance $\mathrm{P}(2.9 \mathrm{mg}, 1.6 \mu \mathrm{mol}, 72 \%$ yield).

${ }^{1} \mathrm{H}_{\text {NMR }}\left(700 \mathrm{MHz}, \mathrm{d}_{6}\right.$-DMSO, $\left.295 \mathrm{~K}\right) \delta: 0.84(\mathrm{~d}, 3 \mathrm{H}), 0.88(\mathrm{~d}, 3 \mathrm{H}), 1.39(\mathrm{~m}, 2 \mathrm{H}), 1.47(\mathrm{~m}, 2 \mathrm{H}), 1.51(\mathrm{~m}, 1 \mathrm{H})$, $1.54(\mathrm{~m}, 2 \mathrm{H}), 1.61(\mathrm{~m}, 3 \mathrm{H}), 1.67-1.77(\mathrm{~m}, 6 \mathrm{H}), 1.78-1.88(\mathrm{~m}, 6 \mathrm{H}), 1.91(\mathrm{~m}, 3 \mathrm{H}), 2.03(\mathrm{~m}, 4 \mathrm{H}), 2.11(\mathrm{~m}, 4 \mathrm{H})$, $2.38(\mathrm{~m}, 1 \mathrm{H}), 2.45(\mathrm{~m}, 1 \mathrm{H}), 2.67(\mathrm{dd}, 1 \mathrm{H}), 2.75(\mathrm{~m}, 3 \mathrm{H}), 2.92(\mathrm{~m}, 2 \mathrm{H}), 3.13(\mathrm{~m}, 2 \mathrm{H}), 3.60(\mathrm{~m}, 2 \mathrm{H}), 3.72(\mathrm{~m}$, $3 \mathrm{H}), 4.15(\mathrm{~m}, 3 \mathrm{H}), 4.23(\mathrm{~m}, 1 \mathrm{H}), 4.30(\mathrm{~m}, 2 \mathrm{H}), 4.43(\mathrm{~m}, 4 \mathrm{H}), 6.77(\mathrm{~d}, 1 \mathrm{H}, \mathrm{J}=8.2 \mathrm{~Hz}), 6.87(\mathrm{~s}, 2 \mathrm{H}), 7.08(\mathrm{~m}$, $2 \mathrm{H}), 7.15(\mathrm{~m}, 3 \mathrm{H}), 7.19(\mathrm{~m}, 3 \mathrm{H}), 7.32(\mathrm{~s}, 2 \mathrm{H}), 7.58(\mathrm{~d}, 1 \mathrm{H}, \mathrm{J}=1.8 \mathrm{~Hz}), 7.69(\mathrm{t}, 1 \mathrm{H}), 7.77(\mathrm{br}, 3 \mathrm{H}), 7.84(\mathrm{~d}$, $1 \mathrm{H}), 7.97(\mathrm{t}, 2 \mathrm{H}), 8.01(\mathrm{t}, 2 \mathrm{H}), 8.18(\mathrm{br}, 3 \mathrm{H}), 8.23(\mathrm{t}, 1 \mathrm{H}), 8.27(\mathrm{~d}, 1 \mathrm{H}), 8.31(\mathrm{~d}, 1 \mathrm{H}), 10.10(\mathrm{~s}, 1 \mathrm{H})$.

${ }^{13} \mathrm{C}$ NMR $\left(175 \mathrm{MHz}, \mathrm{d}_{6}\right.$-DMSO, $\left.295 \mathrm{~K}\right) \delta: 14.65,21.59,21.84,23.13,23.62,24.12,24.67,24.68,26.56$, 27.11, 27.39, 27.90, 29.03, 29.22, 29.70, 30.12, 31.48, 31.57, 31.59, 36.18, 37.50, 38.58, 40.22, 40.70, $41.99,49.88,47.04,50.43,50.48,51.21,51.76,52.30,52.80,54.13,54.29,59.24,59.83,84.28,114.63$, 126.20, 128.04, 129.11, 130.22, 130.33, 137.59, 139.21, 155.10, 156.79, 166.84, 168.64, 170.38, 170.87, 170.92, 170.98, 171.30, 171.93, 172.04, 173.08, 174.19, 174.24.

HRMS (ESI-TOF) Calcd for $\mathrm{C}_{63} \mathrm{H}_{97} \mathrm{IN}_{18} \mathrm{O}_{14} \mathrm{~S}$ : 1488.6197; Found: 1488.6244.

UPLC-MS rt: 2.555 min (Gradient 1).

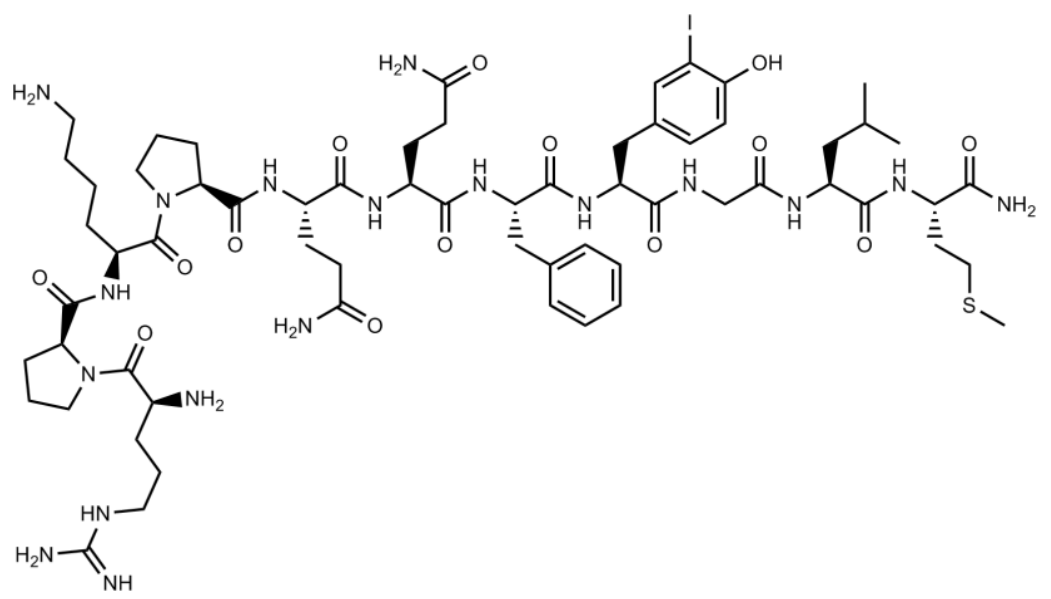



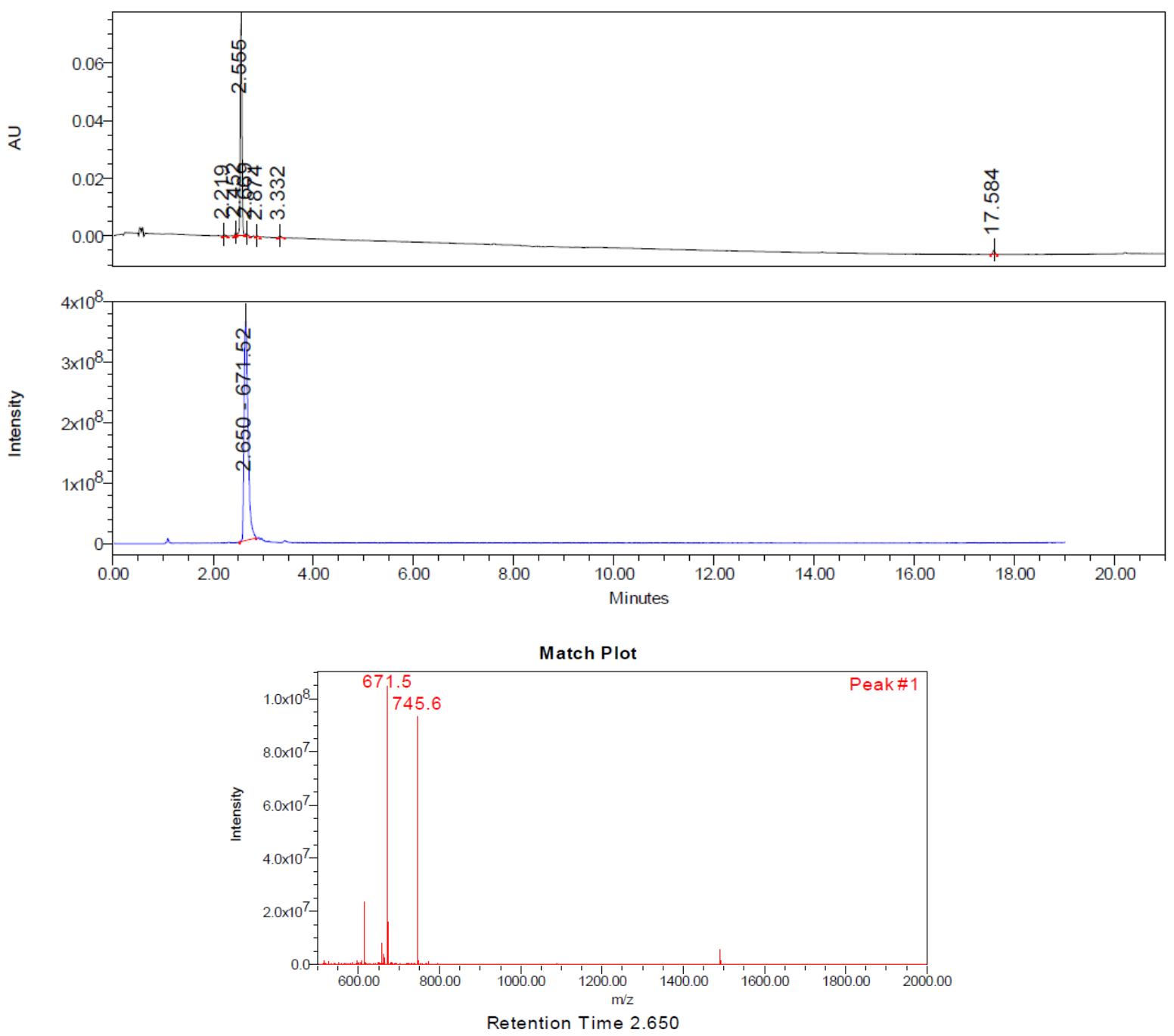


\section{Mono-iodination of GLP-1(7-37)}

GLP-1(7-37) $(40.0 \mathrm{mg}, 11.9 \mu \mathrm{mol})$ was dissolved in $5.6 \mathrm{~mL}$ of DCM and $1.4 \mathrm{~mL}$ of TFA. Then were added dropwise $330 \mu \mathrm{L}$ of a $50 \mathrm{mM}$ iodination stock solution ( $3.0 \mu \mathrm{mol}, 1.4$ eq.) (stock solution freshly prepared: $15.0 \mathrm{mg}$ of Selectfluor were dissolved in $840 \mu \mathrm{L}$ of ACN before the addition of $7.2 \mathrm{mg}$ of Nal). The reaction mixture was allowed to stir at room temperature for 15 minutes. Two other additions of iodination solution ( $2 \times 55 \mu \mathrm{L}, 3 \times 0.25$ eq.) were needed to reach the following final ratio: SM: $4.6 \%$ - MI: 91.3\% - DI: $4.1 \%$. The reaction mixture was then evaporated under reduced pressure (TFA was coevaporated three times with DCM). The crude was dissolved in water/acetonitrile (1/1) before being submitted to HPLC (purification performed on Waters Acquity CSH C18, gradient: see general methods). The collected fractions were lyophilized to afford the desired product [mono-lodo]-GLP-1(7-37) (21.5 mg, $6.2 \mu \mathrm{mol}, 52 \%$ yield).

HRMS (ESI-TOF) Calcd for $\mathrm{C}_{151} \mathrm{H}_{227} \mathrm{IN}_{40} \mathrm{O}_{47}[\mathrm{M}+3 \mathrm{H}]^{3+}$ 1741.2900; Found: 1741.2999.

UPLC-MS rt: 6.217 min (Gradient 1).
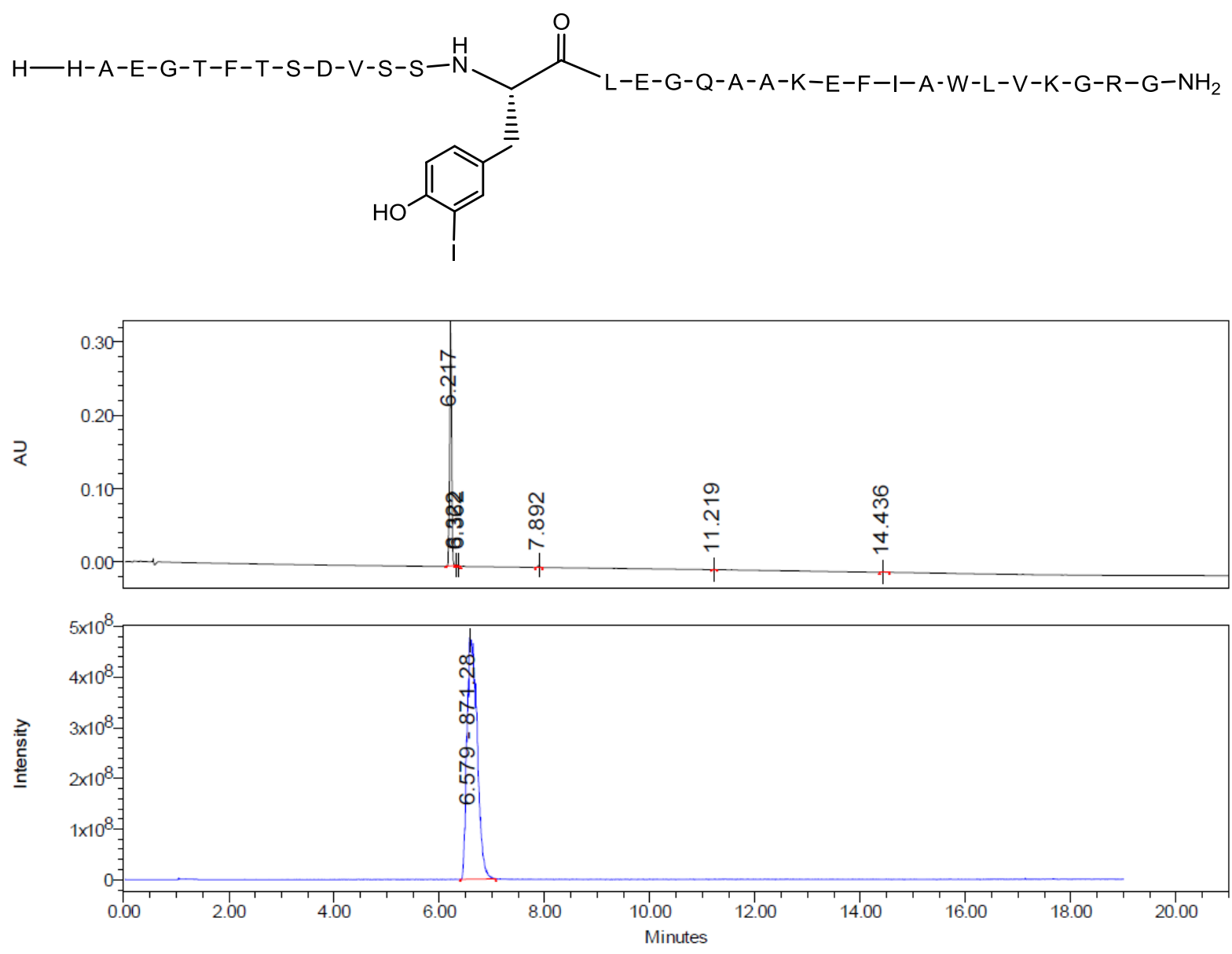


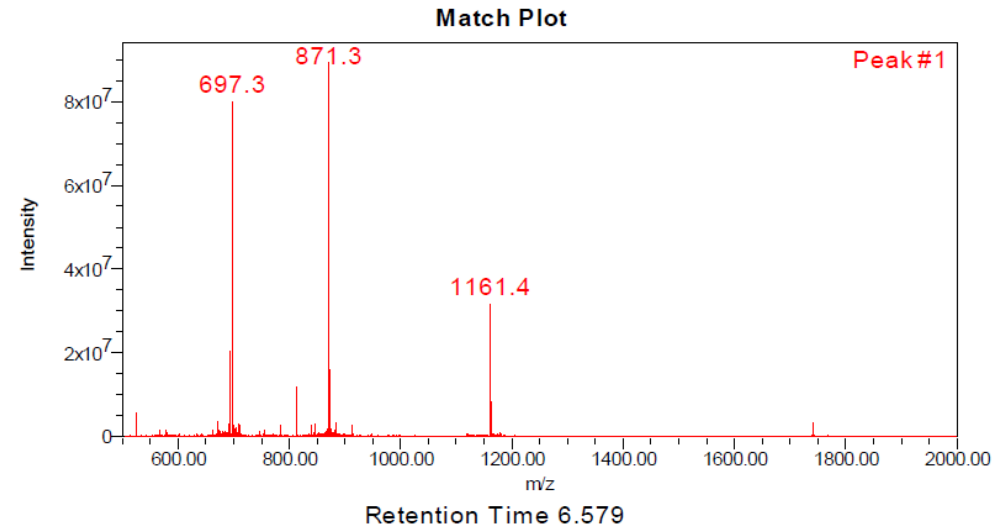

\section{Synthesis of Amino-PEG 2 -Dansyl}

Dansyl chloride (100 mg, $370 \mu \mathrm{mol}$ ) was dissolved in $1.0 \mathrm{~mL}$ of DCM before the addition dropwise of tertbutyl(2-(2-(2-aminoethoxy)ethoxy)ethyl)carbamate $(105 \mu \mathrm{L}, 445 \mu \mathrm{mol})$ previously dissolved in $1 \mathrm{~mL}$ of DCM. The mixture was allowed to stir at room temperature for 2 hours. Reaction was monitored by LCMS. The mixture was then diluted with $4 \mathrm{~mL}$ of brine, washed with water three times before being dried over magnesium sulfate and filtered. Subsequently $450 \mu \mathrm{L}$ of TFA were added to the mixture ( $10 \% \mathrm{v} / \mathrm{v}$ DCM) to induce Boc deprotection. After $15 \mathrm{~min}$, LC-MS showed completion of reaction. The mixture was evaporated under reduced pressure and redissolved in acetonitrile and water before lyophilization to afford the desired product as pale yellow oil (201 mg, $346 \mu \mathrm{mol}, 93 \%$ yield).

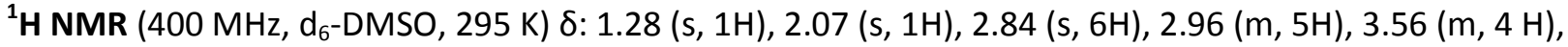
$4.60(\mathrm{~s}, 1 \mathrm{H}), 5.37$ (br s, 3H), $5.75(\mathrm{br} \mathrm{s}, 1 \mathrm{H}), 7.27$ (d, J=7.46 Hz, 1H), $7.61(\mathrm{~m}, 2 \mathrm{H}), 7.72$ (br s, 3H), 7.99 (t, $\mathrm{J}=5.81,5.81 \mathrm{~Hz}, 1 \mathrm{H}), 8.11(\mathrm{dd}, \mathrm{J}=7.34,0.98 \mathrm{~Hz}, 1 \mathrm{H}), 8.30(\mathrm{~d}, \mathrm{~J}=8.68 \mathrm{~Hz}, 1 \mathrm{H}), 8.47(\mathrm{~d}, \mathrm{~J}=8.44 \mathrm{~Hz}, 1 \mathrm{H})$.

${ }^{13} \mathrm{C}$ NMR $\left(150 \mathrm{MHz}, \mathrm{d}_{6}\right.$-DMSO, $\left.300 \mathrm{~K}\right) \delta: 38.54,42.16,45.06$ (2C), 66.58, 69.01, 69.30, 69.43, 115.13, $119.23,123.56,127.75,128.00,128.99,129.03,129.27,136.21,151.17$.

HRMS (ESI-TOF) Calcd for $\mathrm{C}_{18} \mathrm{H}_{27} \mathrm{~N}_{3} \mathrm{O}_{4} \mathrm{~S}$ : 382.1795; Found: 382.1795.

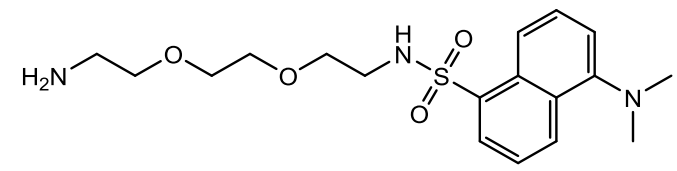




\section{Synthesis of Boronic pinacol ester-PEG 2 -Dansyl (9)}

4-Carboxylphenylboronic acid pinacol ester (31 mg, $125 \mu \mathrm{mol})$, DIEA (45 $\mu \mathrm{L}, 258 \mu \mathrm{mol})$ and propylphosphonic anhydride T3P (75 $\mu \mathrm{L}, 125 \mu \mathrm{mol}$ - 50\% wt. solution in ethyl acetate) were dissolved in $1 \mathrm{~mL}$ of DCM and stirred at room temperature for 5 minutes before the dropwise addition of amino$\mathrm{PEG}_{2}$-Dansyl ( $60 \mathrm{mg}, 104 \mu \mathrm{mol}$ ) and DIEA ( $45 \mu \mathrm{L}, 258 \mu \mathrm{mol}$ ) previously dissolved in $750 \mu \mathrm{L}$ of DCM. The reaction mixture was allowed to stir at room temperature. After $30 \mathrm{~min}, \mathrm{LC}-\mathrm{MS}$ showed completion of reaction. The crude was evaporated under reduced pressure and purified via silica gel chromatography (0->100\% EtOAc/Hept - product eluted around 80\% EtOAc/Hept) to afford the desired product as a yellow oil (39 mg, $64 \mu \mathrm{mol}, 62 \%$ yield).

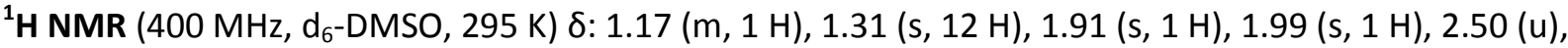
$2.82(\mathrm{~s}, 6 \mathrm{H}), 2.94(\mathrm{q}, \mathrm{J}=5.83,5.83,5.83 \mathrm{~Hz}, 2 \mathrm{H}), 3.28(\mathrm{~m}, 4 \mathrm{H}), 3.45(\mathrm{u}), 4.03$ (q, J=7.17, 7.17, 7.17 Hz, 1 H), 7.24 (d, J=7.46 Hz, 1 H), 7.59 (m, 2 H), 7.74 (m(para), 2 H), 7.83 (m(para), 2 H), 8.11 (dd, J=7.34, 0.98 $\mathrm{Hz}, 1 \mathrm{H}), 7.98(\mathrm{t}, \mathrm{J}=5.81,5.81 \mathrm{~Hz}, 1 \mathrm{H}), 8.28(\mathrm{~d}, \mathrm{~J}=8.68 \mathrm{~Hz}, 1 \mathrm{H}), 8.44(\mathrm{~d}, \mathrm{~J}=8.56 \mathrm{~Hz}, 1 \mathrm{H}), 8.52(\mathrm{t}, \mathrm{J}=5.50$, $5.50 \mathrm{~Hz}, 1 \mathrm{H})$.

${ }^{13} \mathrm{C}$ NMR (150 MHz, $\mathrm{d}_{6}$-DMSO, $\left.300 \mathrm{~K}\right)$ ): 24.65(4C), 39.51, 42.20, 45.03(2C), 68.72, 68.93, 69.29, 69.38, 83.89(2C), 115.05, 119.21, 123.50, 126.47(2C), 127.71, 127.97, 129.01, 129.03, 129.24, 134.21(2C), $136.32,136.80,151,27,165.99$.

The carbon directly bonded to boron could not be detected due to quadrupolar relaxation.

HRMS (ESI-TOF) Calcd for $\mathrm{C}_{31} \mathrm{H}_{43} \mathrm{BN}_{3} \mathrm{O}_{7} \mathrm{~S}[\mathrm{M}+\mathrm{Na}]^{+}$634.2734; Found: 634.2737.

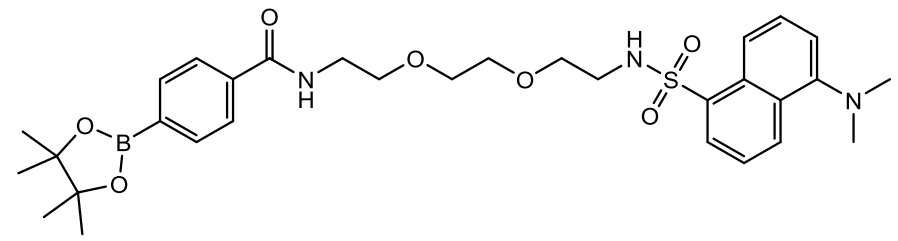

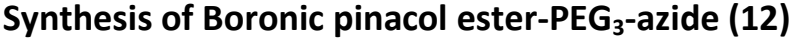

4-carboxylphenylboronic acid pinacol ester ( $200 \mathrm{mg}, 806 \mu \mathrm{mol})$, DIEA (422 $\mu \mathrm{L}, 2.42 \mathrm{mmol}$ ) and propylphosphonic anhydride T3P $(720 \mu \mathrm{L}, 1.21 \mathrm{mmol}-50 \% \mathrm{wt}$. solution in ethyl acetate) were dissolved in $500 \mu \mathrm{L}$ of DCM and was allowed to premix at room temperature for 5 minutes before the dropwise addition of amino-PEG -azide ( $211 \mathrm{mg}, 968 \mu \mathrm{mol}$ ) previously dissolved in $500 \mu \mathrm{L}$ of DCM .

The reaction was stirred at room temperature for 10 minutes. Reaction was monitored by LC-MS. The crude was dissolved in $5 \mathrm{~mL}$ of DCM and $5 \mathrm{~mL}$ of $0.5 \mathrm{M} \mathrm{HCl}$ aqueous solution, washed twice with water $(2 \times 5 \mathrm{~mL})$ before being dried with magnesium sulfate, filtered and evaporated under reduced pressure to afford the desired product as a white powder ( $308 \mathrm{mg}, 687 \mu \mathrm{mol}, 85 \%$ yield).

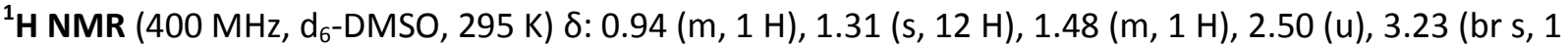
H), 3.44 (br s, 1 H), 3.53 (d, J=4.65 Hz, 9 H), 3.57 (m, 4 H), 7.79 (m(para), 4 H), 8.55 (t, J=5.50, 5.50 Hz, 1 H). 


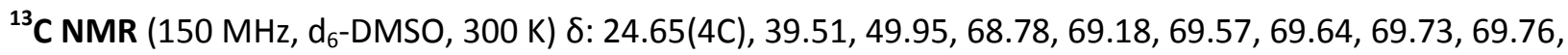
$83.89(2 \mathrm{C}), 126.49(2 \mathrm{C}), 134.21(2 \mathrm{C}), 136.84,166.00$.

The carbon directly bonded to boron could not be detected due to quadrupolar relaxation.

HRMS (ESI-TOF) Calcd for $\mathrm{C}_{21} \mathrm{H}_{34} \mathrm{BN}_{4} \mathrm{O}_{6}[\mathrm{M}+\mathrm{Na}]^{+}$471.2389; Found: 471.2388.

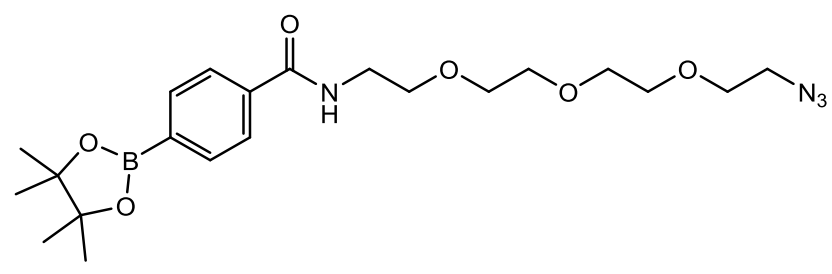

\section{Synthesis of Dansyl-PEG 2 -Leucin Enkephalinamide (10) - Suzuki-Miyaura Cross-coupling}

Preparation of the Pd catalyst was performed as described by Chalker et al $^{2}$. Briefly, 2-amino-4,6dihydroxypyrimidine disodium $(7.6 \mathrm{mg}, 44 \mu \mathrm{mol})$ was dissolved in $440 \mu \mathrm{L}$ of water by stirring for 2 minutes in a water bath preheated to $65^{\circ} \mathrm{C}$. To the resulting solution was added $\mathrm{Pd}(\mathrm{OAc})_{2}(5.0 \mathrm{mg}, 22$ $\mu \mathrm{mol})$. The mixture was stirred vigorously at $65^{\circ} \mathrm{C}$ for 30 minutes to give a homogenous yellow-orange solution. After cooling to room temperature, the stir bar was removed to give the catalyst stock solution $50 \mathrm{mM}$ in $\mathrm{Pd}(\mathrm{II})$.

In a $1.5 \mathrm{~mL}$ Eppendorf tube, [mono-lodo]-Leucine Enkephalinamide $(1.0 \mathrm{mg}, 1.50 \mu \mathrm{mol})$ was dissolved in $75 \mu \mathrm{L}$ of water and $75 \mu \mathrm{L}$ of dioxane before the addition of glycerol $(50 \mu \mathrm{L}), \mathrm{K}_{2} \mathrm{HPO}_{4}(1 \mathrm{M}$ aqueous stock

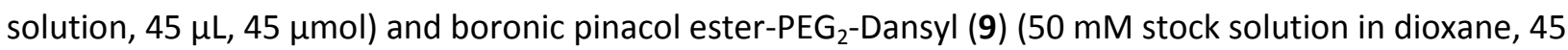
$\mu \mathrm{L}, 2.25 \mu \mathrm{mol})$. Prior and after the addition of $\mathrm{Pd}(\mathrm{OAc})_{2} \cdot \mathrm{L}_{2}(50 \mathrm{mM}$ aqueous stock solution, $75 \mu \mathrm{L}, 3.75$

$\mu \mathrm{mol})$ the mixture was bubbled with argon for 10 minutes. The resulting solution was capped and stirred at $38^{\circ} \mathrm{C}$ for 12 hours on an Eppendorf Thermomixer (1300 rpm). The reaction was monitored by LC-MS. The crude mixture was quenched with $1 \mathrm{~mL}$ of a $1 \mathrm{M} \mathrm{HCl}$ aqueous solution before being submitted to HPLC (purification performed on Agilent Zorbax Rx C18, gradient: see general methods). The collected fractions were lyophilized to afford the desired product Dansyl- $P E G_{2}$-Leucine Enkephalinamide (0.55 mg, $0.53 \mu \mathrm{mol}, 35 \%$ yield).

HRMS (ESI-TOF) Calcd for $\mathrm{C}_{53} \mathrm{H}_{67} \mathrm{~N}_{9} \mathrm{O}_{11} \mathrm{~S}: 1038.4754$; Found: 1038.4753.

UPLC-MS rt: 6.793 min (Gradient 1).

Max Abs/Em - / $506 \mathrm{~nm}$ (absorption <400 nm cannot be measured with our device) 

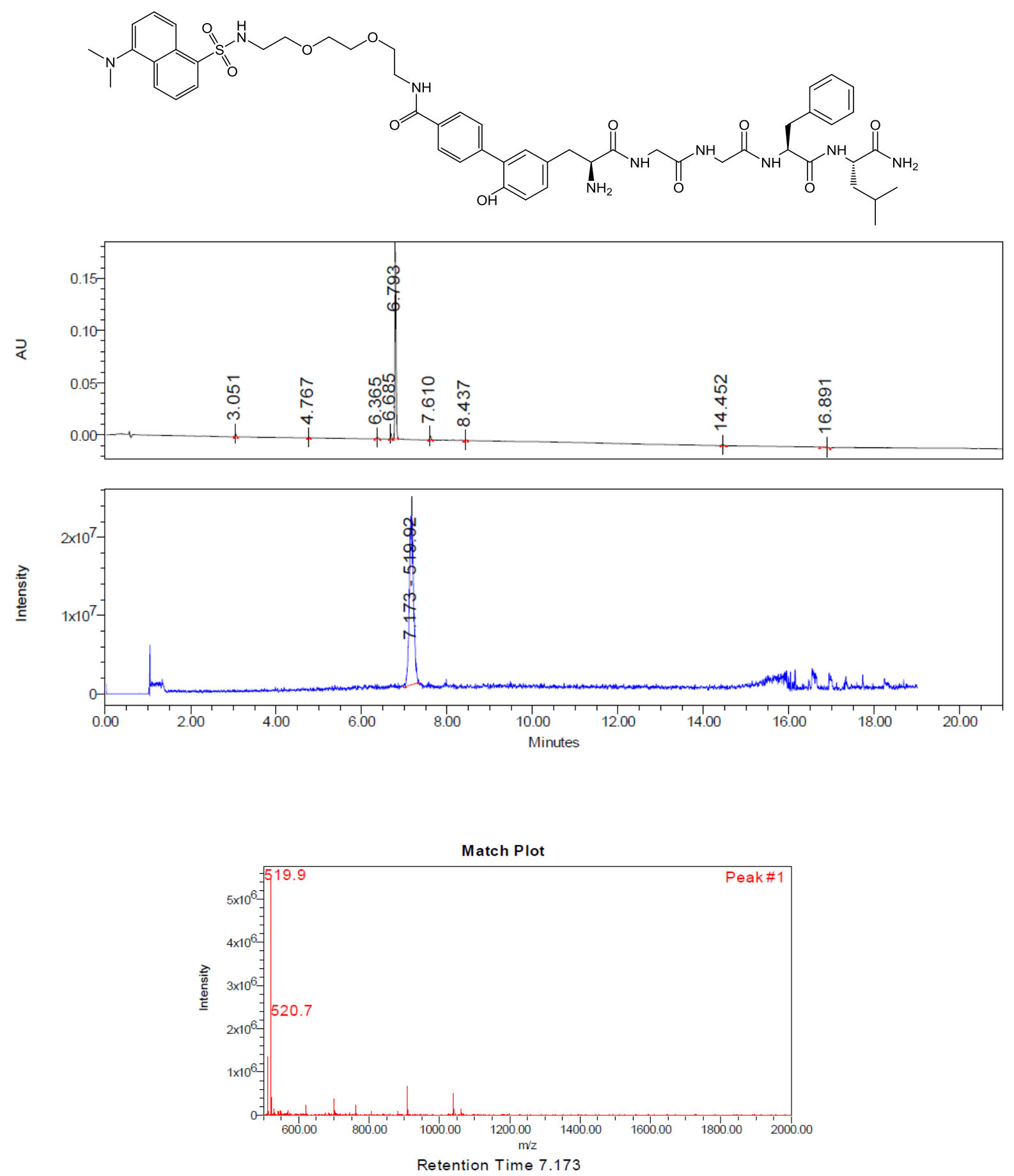
Fluorescence spectra of Dansyl-PEG 2 -Leucin Enkephalinamide in dioxane

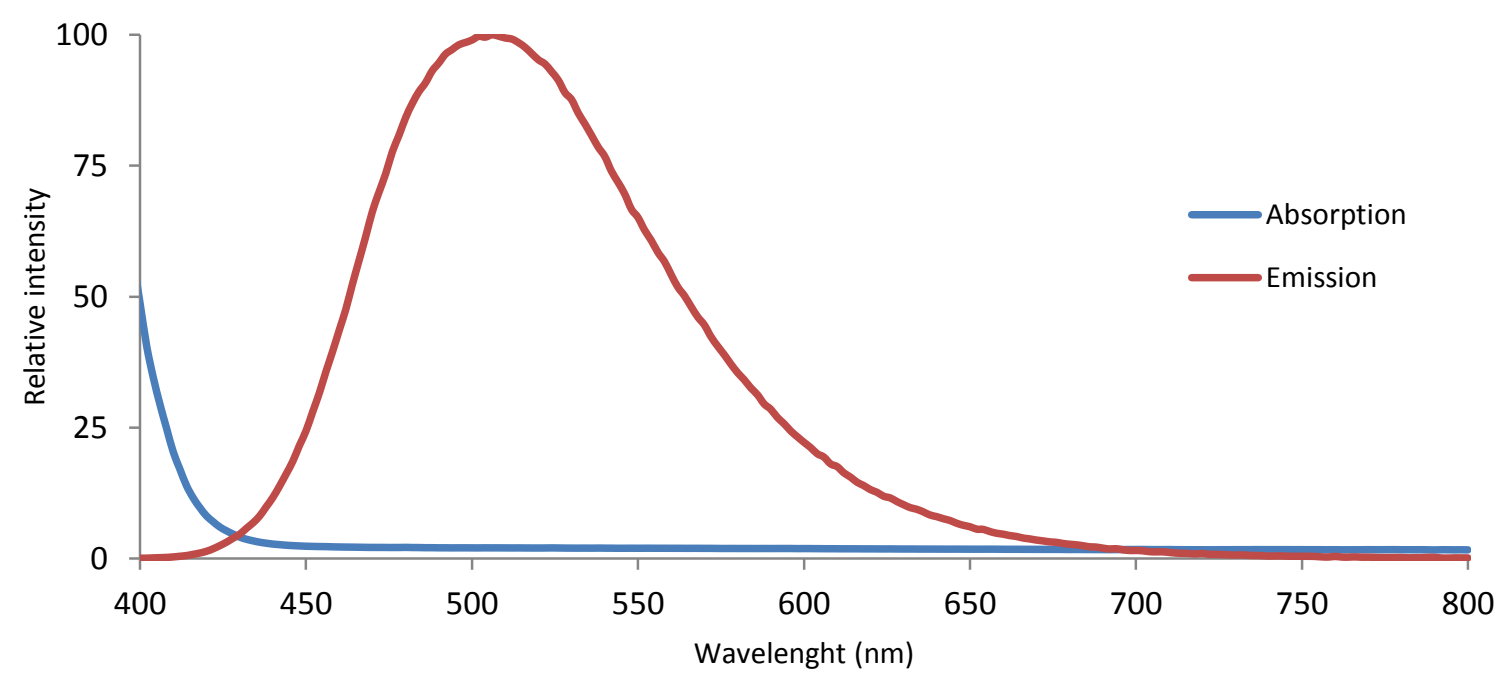

\section{Synthesis of Azido-PEG ${ }_{3}$-ACP fragment (65-74) - Suzuki-Miyaura Cross-coupling}

In a $1.5 \mathrm{~mL}$ Eppendorf tube, [mono-lodo]-ACP fragment (65-74) (2.43 mg, $2.05 \mu \mathrm{mol})$ was dissolved in $100 \mu \mathrm{L}$ of water and $100 \mu \mathrm{L}$ of dioxane before the addition of glycerol $(50 \mu \mathrm{L}), \mathrm{K}_{2} \mathrm{HPO}_{4}(1 \mathrm{M}$ aqueous stock solution, $50 \mu \mathrm{L}, 50 \mu \mathrm{mol}$ ) and boronic pinacol ester- $\mathrm{PEG}_{3}$-azide (12) (50 mM stock solution in dioxane, $75 \mu \mathrm{L}, 3.75 \mu \mathrm{mol})$. Prior and after the addition of $\mathrm{Pd}(\mathrm{OAc})_{2} \cdot \mathrm{L}_{2}(50 \mathrm{mM}$ aqueous stock solution, $80 \mu \mathrm{L}, 4.0 \mu \mathrm{mol}$ ) the mixture was bubbled with argon for 10 minutes. The resulting solution was capped and stirred at $38^{\circ} \mathrm{C}$ for 12 hours on an Eppendorf Thermomixer (1300 rpm). The reaction was monitored by LC-MS. The crude mixture was quenched with $1 \mathrm{~mL}$ of a $1 \mathrm{M} \mathrm{HCl}$ aqueous solution before being submitted to HPLC (purification performed on Agilent Zorbax Rx C18, gradient: see general methods). The collected fractions were lyophilized to afford the desired product Azido-PEG - ACP fragment (65-74) (1.68 mg, $1.22 \mu \mathrm{mol}, 59 \%$ yield).

HRMS (ESI-TOF) Calcd for $\mathrm{C}_{62} \mathrm{H}_{95} \mathrm{~N}_{17} \mathrm{O}_{19}$ : 1380.6917; Found: 1380.6899.

UPLC-MS rt: 4.950 min (Gradient 1).<smiles>CC[C@H](C)[C@H](NC(=O)[C@H](C)NC(=O)[C@H](C)NC(=O)[C@H](CCC(N)=O)NC(=O)[C@@H](N)C(C)C)C(=O)N[C@@H](CC(=O)O)C(=O)N[C@@H](Cc1ccc(O)c(-c2ccc(C(=O)NCCOCCOCCOCCN)cc2)c1)C(=O)N[C@@H](C(=O)N[C@@H](CC(N)=O)C(=O)NCC(N)=O)[C@H](C)CC</smiles> 

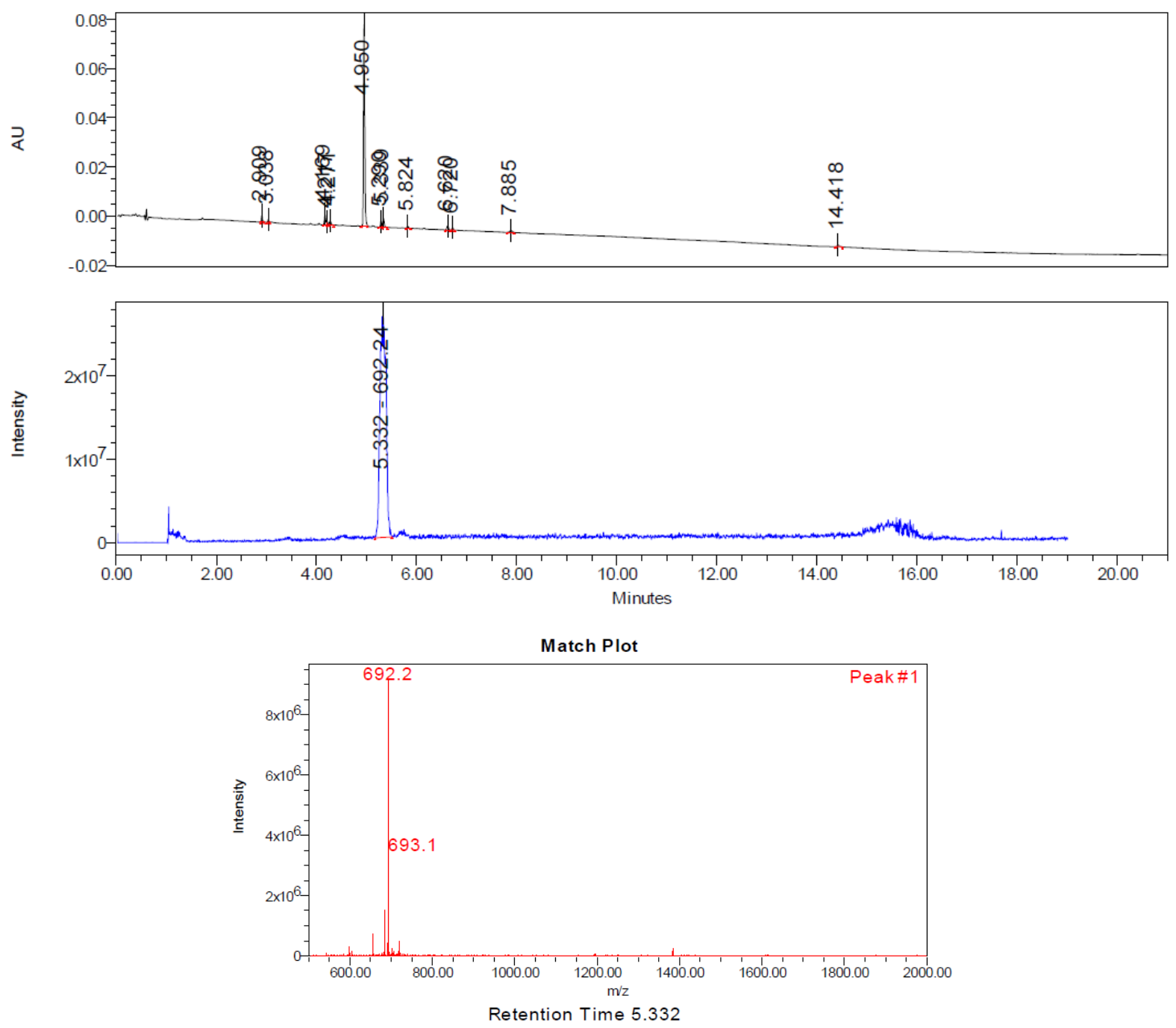

Synthesis of Alexa Fluor 488-PEG - -ACP fragment (65-74) (13) - CuAAC

Azido- $\mathrm{PEG}_{3}$ - ACP fragment (65-74) $(1.68 \mathrm{mg}, 1.22 \mu \mathrm{mol})$ was dissolved in $200 \mu \mathrm{L}$ of water and $100 \mu \mathrm{L}$ of tBuOH before the addition of Alkyne-Alexa Fluor $488(1.0 \mathrm{mg}, 1.29 \mu \mathrm{mol})$. Copper sulfate $(50 \mathrm{mM}$ stock solution in water, $3 \mu \mathrm{L}, 0.15 \mu \mathrm{mol})$, Cu-ligand THPTA ${ }^{3}$ (200 mM stock solution in water, $3 \mu \mathrm{L}, 0.60 \mu \mathrm{mol}$ ) and ascorbic acid ( $100 \mathrm{mM}$ stock solution in water, $7.5 \mu \mathrm{L}, 0.75 \mu \mathrm{mol}$ ) were premixed together before being added to the reaction mixture. The resulting solution was stirred at $38^{\circ} \mathrm{C}$ in a Thermomixer $(1300$ $\mathrm{rpm}$ ) for 12 hours. The reaction was monitored by LC-MS. The crude mixture was dissolved in water/ACN before being submitted to HPLC (purification performed on Agilent Zorbax Rx C18, gradient: see general methods). The collected fractions were lyophilized to afford the desired product as an orange powder Alexa Fluor488-PEG 3 -ACP fragment (65-74) (1.48 mg, $0.76 \mu \mathrm{mol}, 62 \%$ yield).

HRMS (ESI-TOF) Calcd for $\mathrm{C}_{86} \mathrm{H}_{112} \mathrm{~N}_{20} \mathrm{O}_{29} \mathrm{~S}_{2}[\mathrm{M}+2 \mathrm{H}]^{2+}$ 977.8760; Found: 977.8803 .

UPLC-MS rt: 5.009 min (Gradient 1).

Max Abs/Em 494/524 nm. 

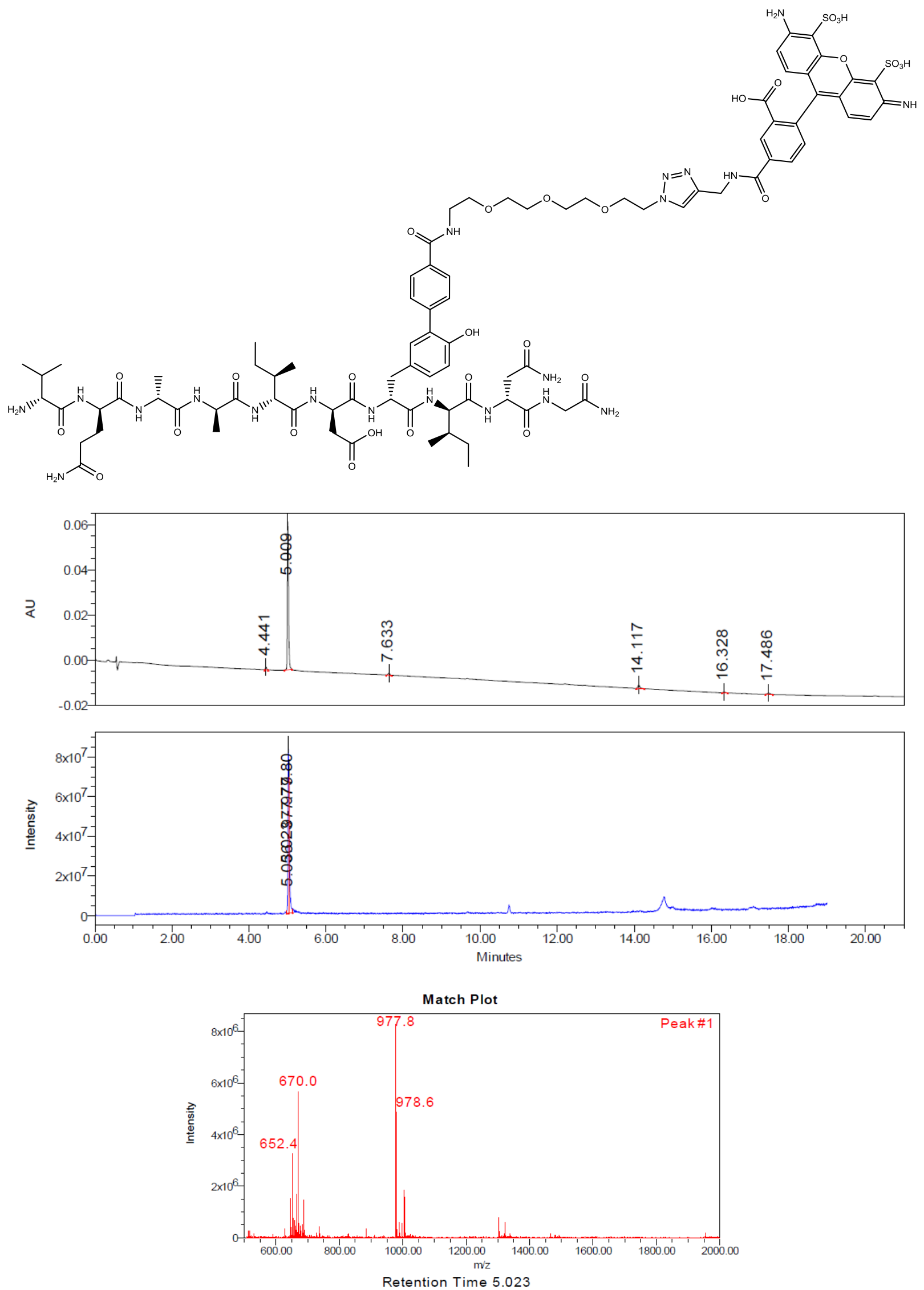
Fluorescence spectra of AF488-PEG ${ }_{3}$-ACP fragment (65-74) in PBS

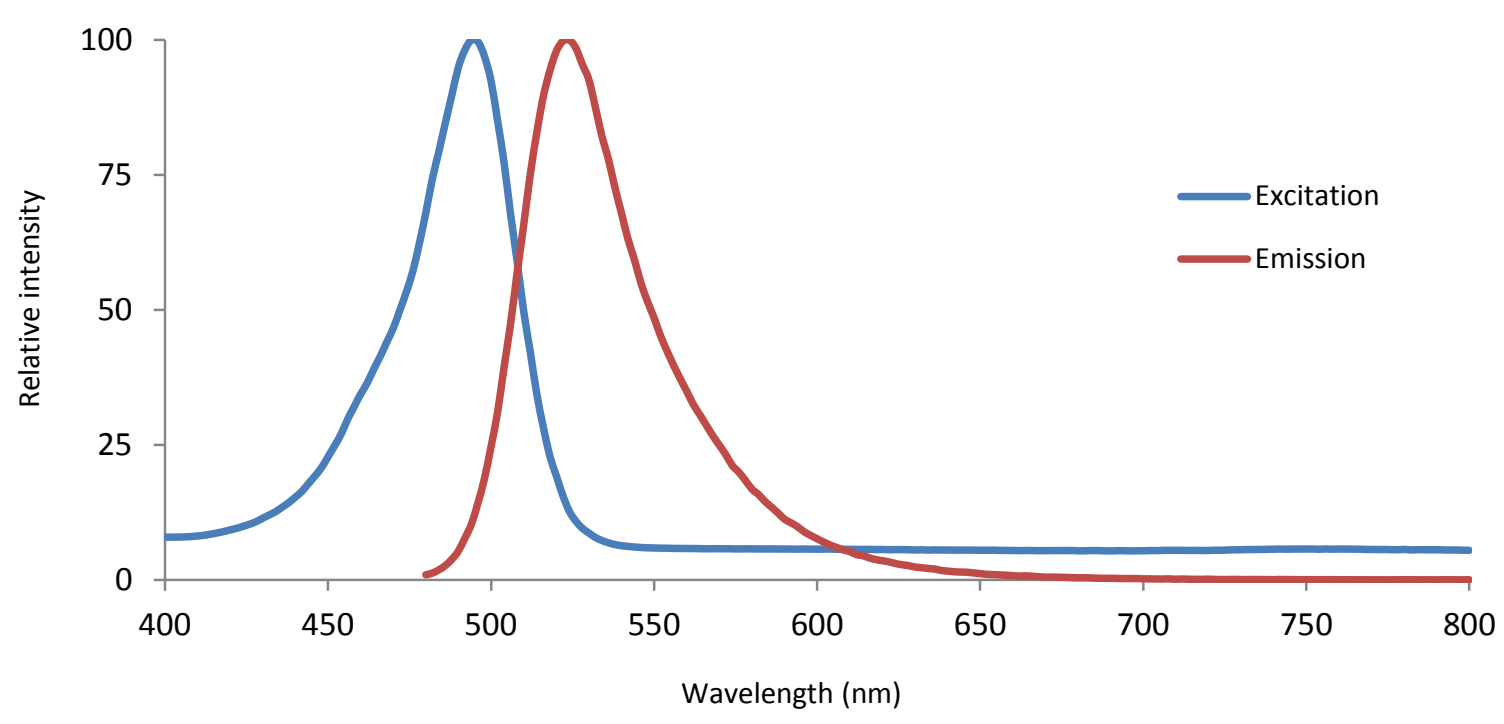


NMR Spectra and proton assignment of mono-iodinated peptides

[mono-iodo] Leucine Enkephalinamide

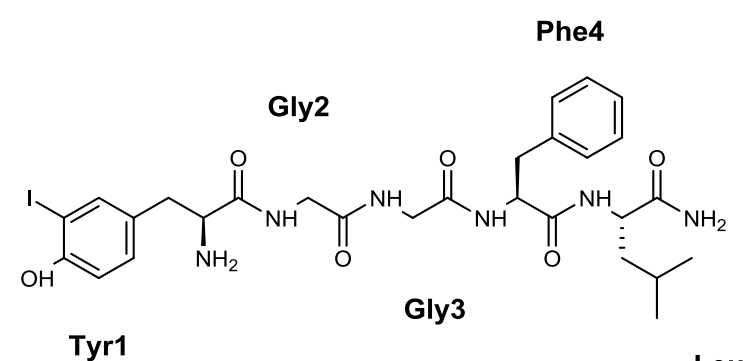

${ }^{1} \mathrm{H}$ NMR (500 MHz, $\mathrm{d}_{6}$-DMSO, $300 \mathrm{~K}$ ) and ${ }^{13} \mathrm{C}$ NMR (125 MHz, $\mathrm{d}_{6}$-DMSO, $300 \mathrm{~K}$ ).

\begin{tabular}{|c|c|c|}
\hline & ${ }^{1} \mathrm{H}$ & ${ }^{13} \mathrm{C}$ \\
\hline $\mathrm{NH}_{3}{ }^{+}$ & broad & - \\
\hline$\alpha$ & 3.46 & 55.77 \\
\hline$\beta$ & $2.86 / 2.51$ & 38.53 \\
\hline$\gamma$ & - & 130.62 \\
\hline$\delta 1$ & 7.53 & 139.22 \\
\hline$\delta 2$ & 7.03 & 130.40 \\
\hline$\varepsilon 1$ & - & 84.36 \\
\hline$\varepsilon 2$ & 6.78 & 114.64 \\
\hline$\zeta$ & - & 155.08 \\
\hline$\zeta-\mathrm{OH}$ & $\sim 10.1$ (broad) & - \\
\hline $\mathrm{C}^{\prime}$ & - & 173.76 \\
\hline Gly-2 & 8.30 & - \\
\hline$\alpha$ & 3.73 & 42.02 \\
\hline $\mathrm{C}^{\prime}$ & - & 169.10 \\
\hline Gly-3 & 8.12 & - \\
\hline$\alpha$ & $3.73 / 3.61$ & 41.90 \\
\hline
\end{tabular}




\begin{tabular}{|c|c|c|}
\hline$C^{\prime}$ & - & 168.75 \\
\hline Phe-4 & 8.08 & - \\
\hline$\alpha$ & 4.50 & 54.09 \\
\hline$\beta$ & $3.04 / 2.80$ & 37.28 \\
\hline$\gamma$ & - & 137.78 \\
\hline$\delta$ & 7.25 & 129.17 \\
\hline$\varepsilon$ & 7.25 & 128.04 \\
\hline$\zeta$ & 7.18 & 126.26 \\
\hline$C^{\prime}$ & - & 170.69 \\
\hline Leu-5 & 7.95 & - \\
\hline$\alpha$ & 4.20 & 50.99 \\
\hline$\beta$ & 1.47 & 40.80 \\
\hline$\gamma$ & 1.57 & 24.19 \\
\hline$\delta$ & 0.88 & 23.02 \\
\hline$\delta^{\prime}$ & 0.83 & 21.56 \\
\hline$C^{\prime}$ & - & 173.88 \\
\hline $\mathrm{NH}_{2}$ & $7.08 / 6.97$ & - \\
\hline
\end{tabular}




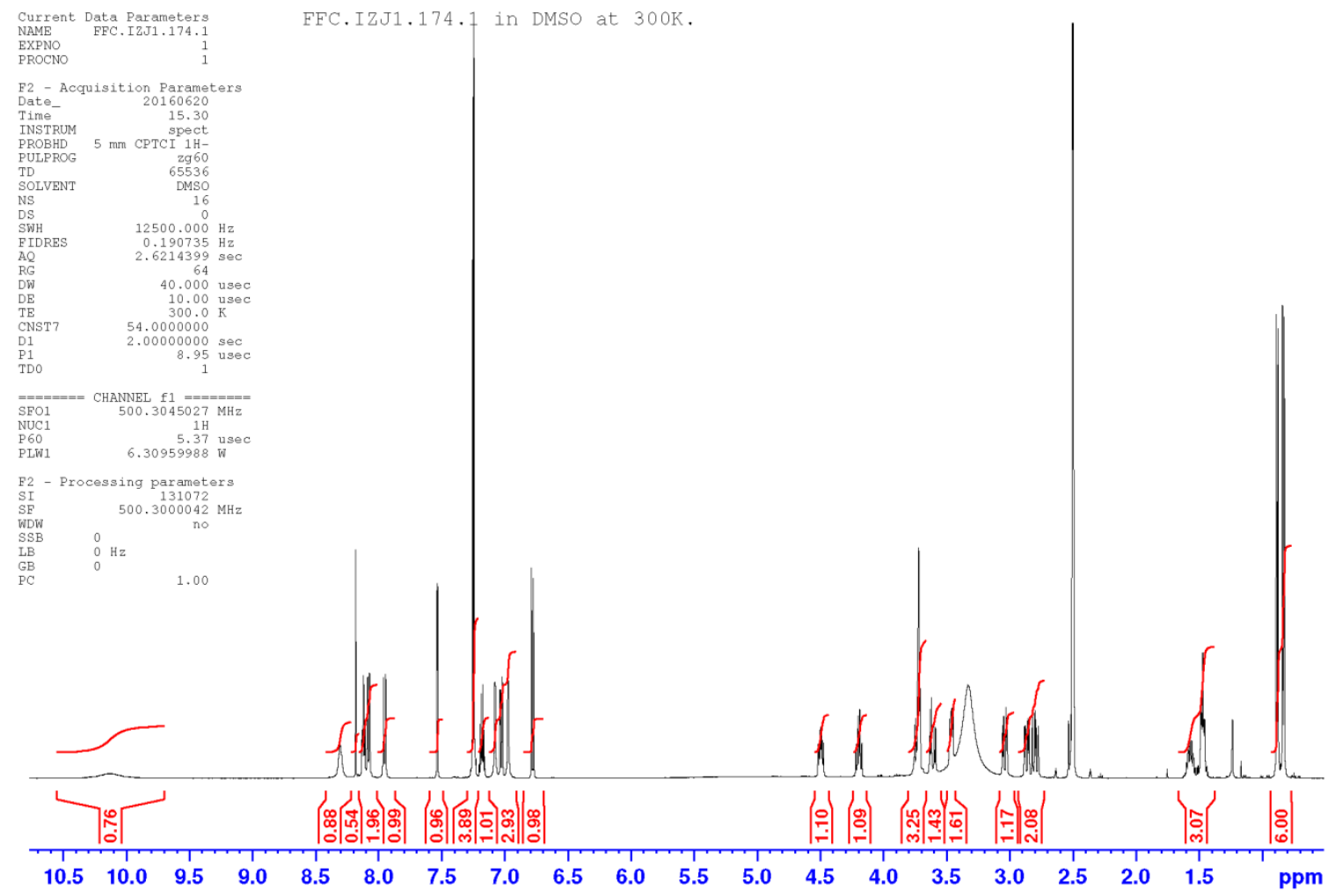




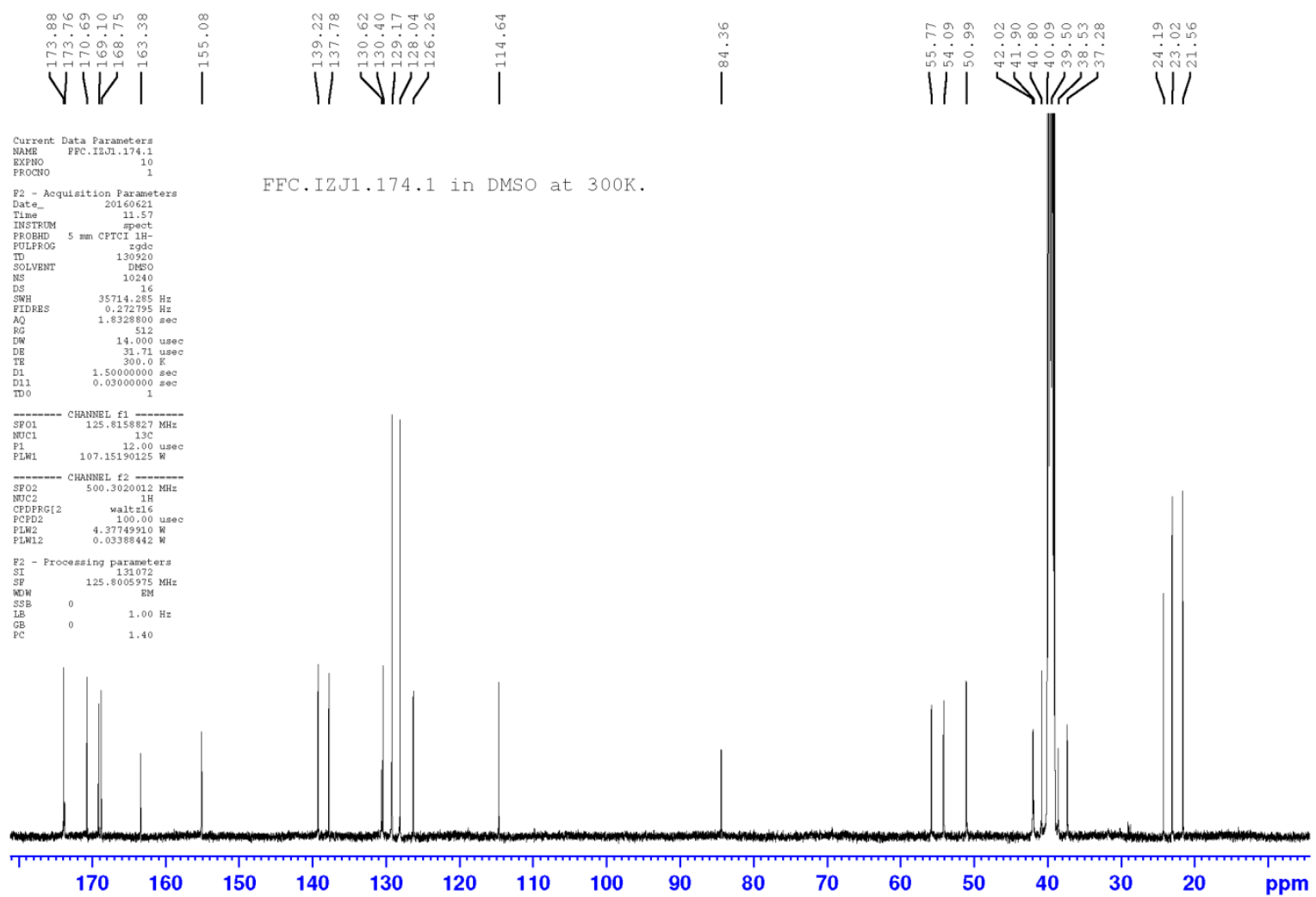

\section{Cyclo(RGD[mono-iodo]yK)}

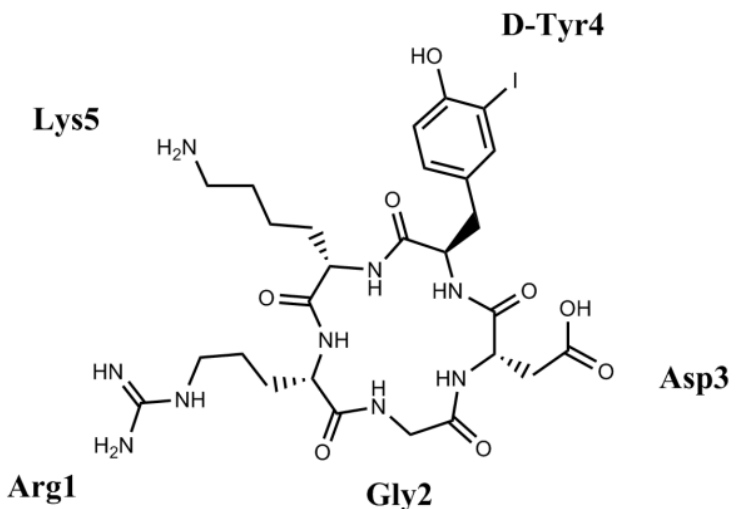

${ }^{1} \mathrm{H}$ NMR (700 MHz, $\mathrm{d}_{6}$-DMSO, $308 \mathrm{~K}$ ) and ${ }^{13} \mathrm{C}$ NMR (175 MHz, $\mathrm{d}_{6}$-DMSO, $308 \mathrm{~K}$ ).

Chemical shifts are referenced to the solvent signals $\left({ }^{1} \mathrm{H}: 2.50 \mathrm{ppm},{ }^{13} \mathrm{C}: 39.50 \mathrm{ppm}\right)$. At $308 \mathrm{~K}$ a better dispersion of the amide signals (Asp-3 and Lys-5) has been obtained. 


\begin{tabular}{|c|c|c|}
\hline & ${ }^{1} \mathrm{H}$ & ${ }^{13} \mathrm{C}$ \\
\hline \multicolumn{3}{|l|}{ Arg-1 } \\
\hline $\mathrm{NH}$ & 7.61 & - \\
\hline$\alpha$ & 4.16 & 51.84 \\
\hline$\beta$ & $1.71 / 1.48$ & 28.48 \\
\hline$\gamma$ & 1.37 & 25.11 \\
\hline$\delta$ & 3.09 & 40.26 \\
\hline$\varepsilon$ & 7.52 & - \\
\hline$\zeta$ & - & 156.61 \\
\hline$C^{\prime}$ & - & 171.10 \\
\hline \multicolumn{3}{|l|}{ Gly-2 } \\
\hline $\mathrm{NH}$ & 8.44 & - \\
\hline$\alpha$ & $4.04 / 3.24$ & 43.22 \\
\hline $\mathrm{C}^{\prime}$ & - & 169.45 \\
\hline \multicolumn{3}{|l|}{ Asp-3 } \\
\hline $\mathrm{NH}$ & 8.09 & - \\
\hline$\alpha$ & 4.64 & 48.81 \\
\hline$\beta$ & $2.71 / 2.39$ & 35.04 \\
\hline$\gamma$ & $(\mathrm{OH}: ~ 12.2)$ & 171.52 \\
\hline $\mathrm{C}^{\prime}$ & - & 169.93 \\
\hline \multicolumn{3}{|l|}{ D-Tyr-4 } \\
\hline $\mathrm{NH}$ & 8.00 & - \\
\hline$\alpha$ & 4.34 & 54.51 \\
\hline$\beta$ & $2.78 / 2.69$ & 35.89 \\
\hline$\gamma$ & - & 129.81 \\
\hline$\delta 1$ & 7.44 & 138.93 \\
\hline
\end{tabular}




\begin{tabular}{|c|c|c|}
\hline$\delta 2$ & 6.97 & 130.21 \\
\hline$\varepsilon 1$ & - & 84.14 \\
\hline$\varepsilon 2$ & 6.77 & 114.61 \\
\hline$\zeta$ & - & 155.02 \\
\hline$\zeta-\mathrm{OH}$ & 10.10 & - \\
\hline$C^{\prime}$ & - & 170.54 \\
\hline \multicolumn{3}{|l|}{ Lys-5 } \\
\hline $\mathrm{NH}$ & 8.11 & - \\
\hline$\alpha$ & 3.94 & 54.38 \\
\hline$\beta$ & $1.58 / 1.43$ & 30.62 \\
\hline$\gamma$ & 1.10 & 22.42 \\
\hline$\delta$ & 1.46 & 26.41 \\
\hline$\varepsilon$ & 2.71 & 38.62 \\
\hline$\zeta$ & 7.69 & - \\
\hline $\mathrm{C}^{\prime}$ & - & 171.82 \\
\hline
\end{tabular}




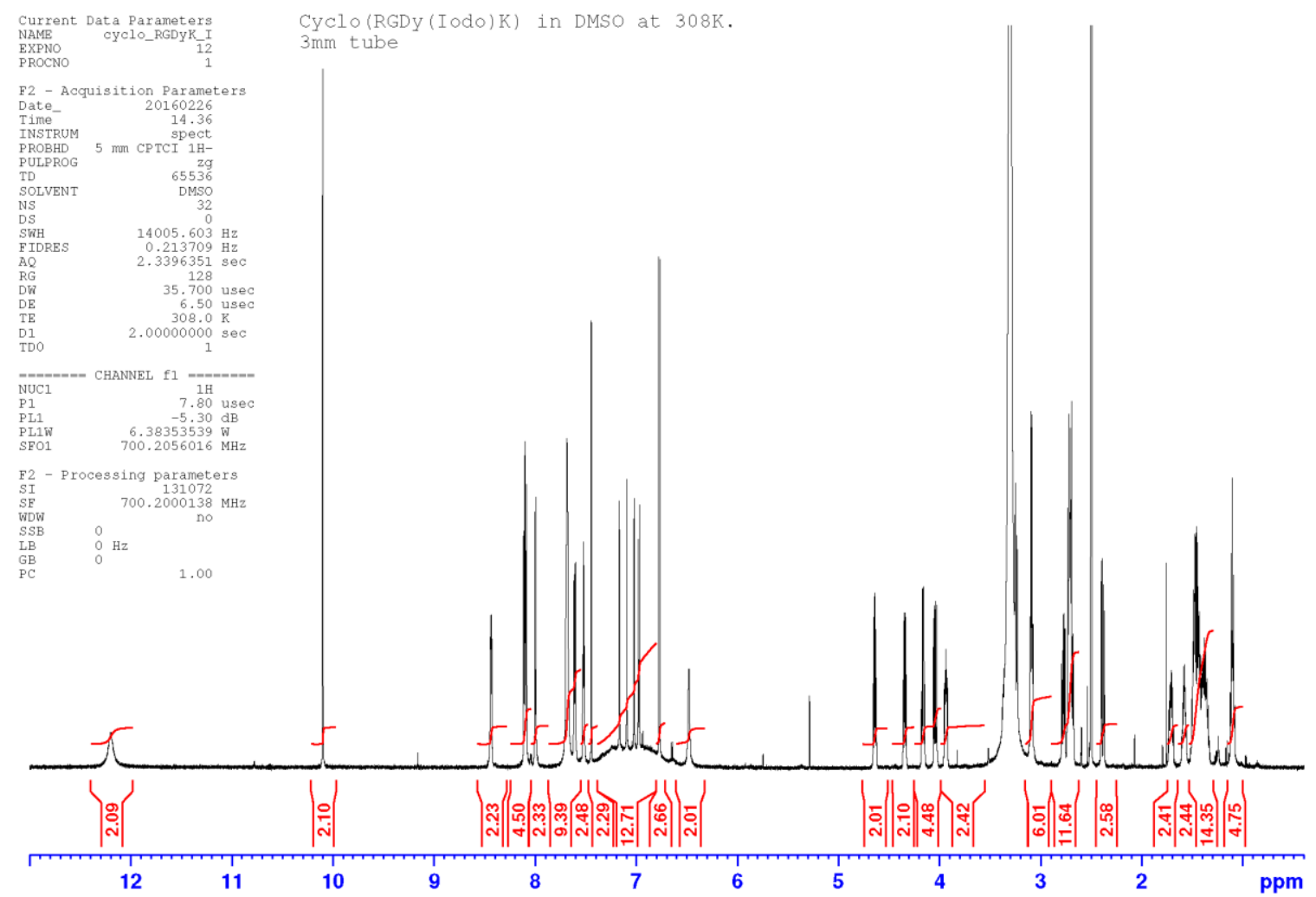




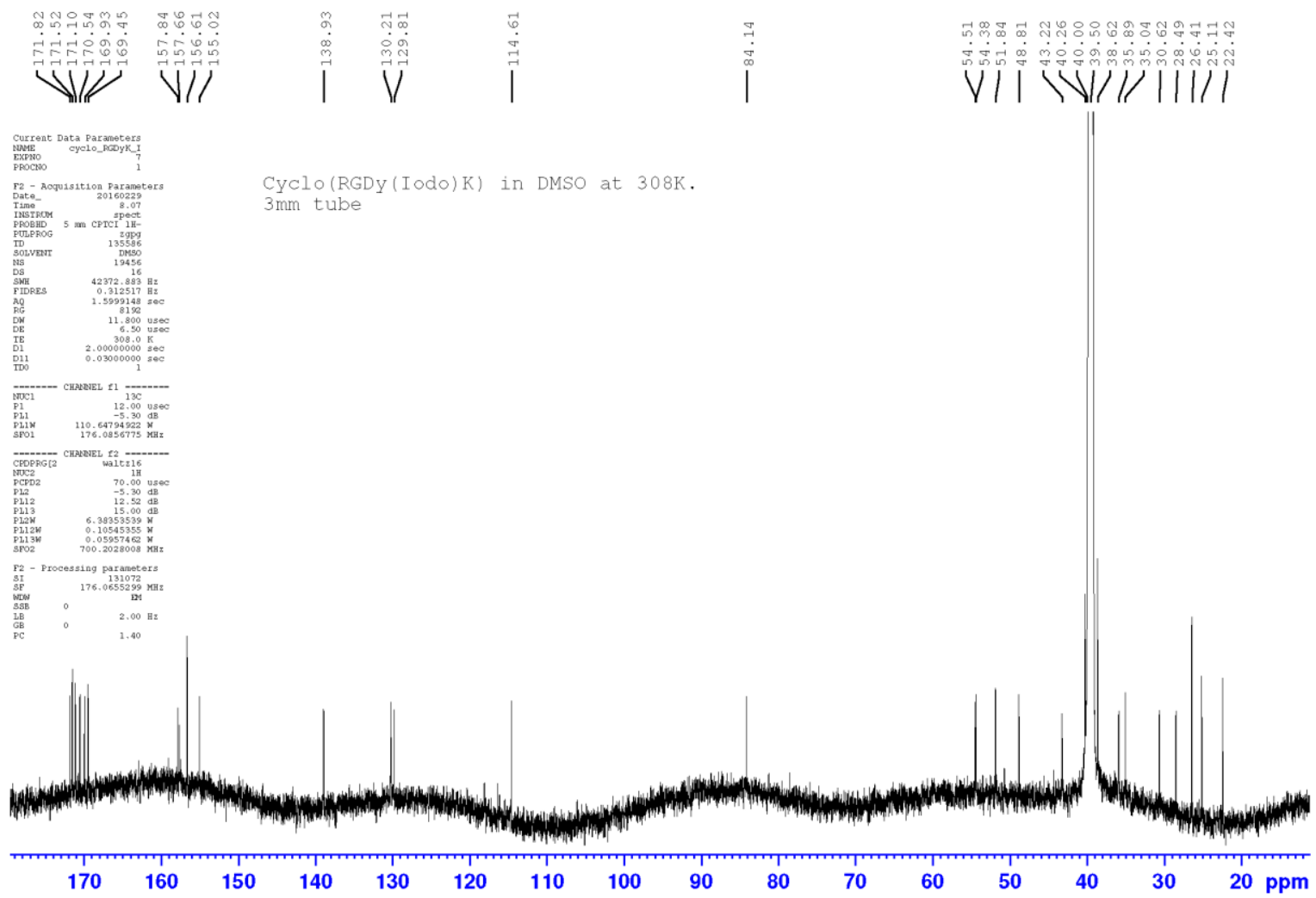


[mono-lodo-Tyr ${ }^{0}$ ]-Bradykinin

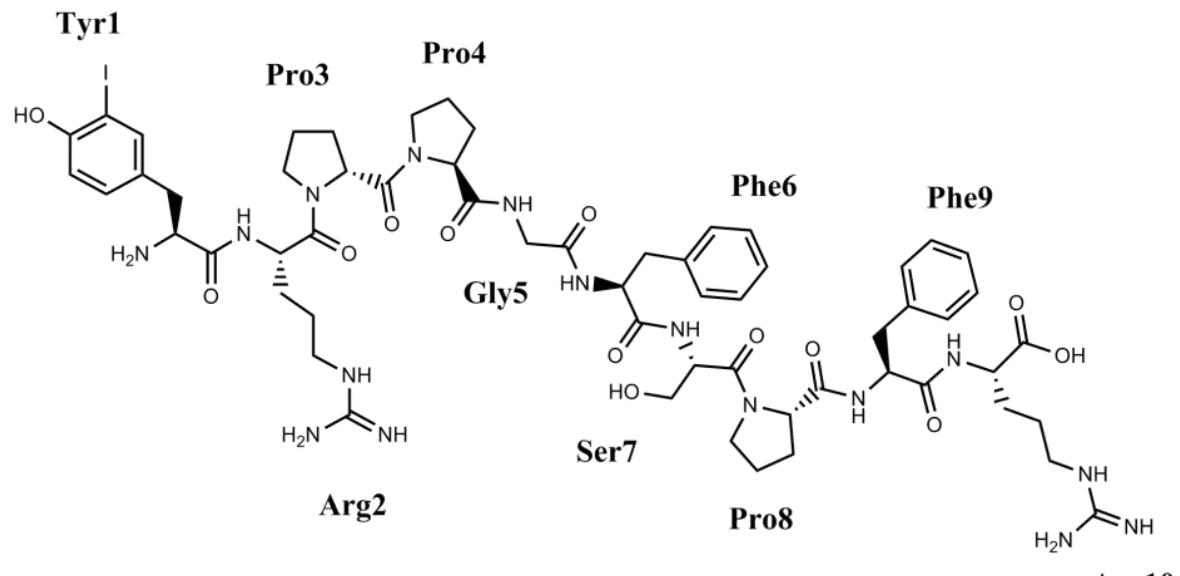

${ }^{1} \mathrm{H}$ NMR (500 MHz, $\mathrm{d}_{6}$-DMSO, $300 \mathrm{~K}$ ) and ${ }^{13} \mathrm{C}$ NMR (125 MHz, $\mathrm{d}_{6}$-DMSO, $300 \mathrm{~K}$ ).

\begin{tabular}{|c|c|c|}
\hline & $\overline{1} \mathrm{H}$ & ${ }^{13} \mathrm{C}$ \\
\hline \multicolumn{3}{|l|}{ Tyr-1 } \\
\hline $\mathrm{NH}_{3}{ }^{+}$ & 8.08 & - \\
\hline$\alpha$ & 3.99 & 53.12 \\
\hline$\beta$ & $2.89 / 2.79$ & 35.44 \\
\hline$\gamma$ & - & 126.90 \\
\hline$\delta 1$ & 7.51 & 139.39 \\
\hline$\delta 2$ & 7.00 & 130.71 \\
\hline$\varepsilon 1$ & - & 84.78 \\
\hline$\varepsilon 2$ & 6.78 & 114.66 \\
\hline$\zeta$ & - & 155.84 \\
\hline$\zeta-\mathrm{OH}$ & 10.31 & - \\
\hline$\overline{C^{\prime}}$ & - & 167.52 \\
\hline \multicolumn{3}{|l|}{ Arg-2 } \\
\hline $\mathrm{NH}$ & 8.66 & - \\
\hline$\alpha$ & 4.49 & 49.98 \\
\hline
\end{tabular}




\begin{tabular}{|c|c|c|}
\hline$\beta$ & $1.69 / 1.50$ & 28.54 \\
\hline$\gamma$ & 1.53 & 24.41 \\
\hline$\delta$ & 3.08 & 40.47 \\
\hline$\varepsilon$ & 7.61 & - \\
\hline$\zeta$ & - & 156.73 \\
\hline$C^{\prime}$ & - & (a) \\
\hline \multicolumn{3}{|l|}{ Pro-3 } \\
\hline$\alpha$ & 4.58 & 57.63 \\
\hline$\beta$ & $2.18 / 1.84$ & 27.87 \\
\hline$\gamma$ & 1.87 & 24.49 \\
\hline$\delta$ & $3.60 / 3.48$ & 46.90 \\
\hline$C^{\prime}$ & - & 169.63 \\
\hline \multicolumn{3}{|l|}{ Pro-4 } \\
\hline$\alpha$ & 4.26 & 59.44 \\
\hline$\beta$ & $2.00 / 1.81$ & 29.05 \\
\hline$\gamma$ & $1.93 / 1.89$ & 24.41 \\
\hline$\delta$ & $3.66 / 3.58$ & 46.81 \\
\hline$C^{\prime}$ & - & 171.77 \\
\hline \multicolumn{3}{|l|}{ Gly-5 } \\
\hline $\mathrm{NH}$ & 7.96 & - \\
\hline$\alpha$ & 3.63 & 41.67 \\
\hline $\mathrm{C}^{\prime}$ & - & 168.48 (or 168.50 ) (a) \\
\hline \multicolumn{3}{|l|}{ Phe-6 } \\
\hline $\mathrm{NH}$ & 7.93 & - \\
\hline$\alpha$ & 4.57 & 53.38 \\
\hline$\beta$ & $2.96 / 2.73$ & 37.68 \\
\hline
\end{tabular}




\begin{tabular}{|c|c|c|}
\hline$\gamma$ & - & 137.57 \\
\hline$\delta$ & 7.19 & 129.13 \\
\hline$\varepsilon$ & 7.24 & 128.02 \\
\hline$\zeta$ & 7.18 & 126.29 \\
\hline$C^{\prime}$ & - & $170.96(b)$ \\
\hline \multicolumn{3}{|l|}{ Ser-7 } \\
\hline $\mathrm{NH}$ & 8.31 & - \\
\hline$\alpha$ & 4.58 & \\
\hline$\beta$ & 3.63 & 61.73 \\
\hline$\beta-\mathrm{OH}$ & 5.36 & - \\
\hline$C^{\prime}$ & - & (a) \\
\hline \multicolumn{3}{|l|}{ Pro-8 } \\
\hline$\alpha$ & 4.30 & 59.60 \\
\hline$\beta$ & $1.88 / 1.60$ & 28.77 \\
\hline$\gamma$ & $1.70 / 1.43$ & 23.72 \\
\hline$\delta$ & $3.61 / 3.51$ & 46.90 \\
\hline$C^{\prime}$ & - & 170.84 \\
\hline \multicolumn{3}{|l|}{ Phe-9 } \\
\hline $\mathrm{NH}$ & 7.70 & - \\
\hline$\alpha$ & 4.54 & 53.45 \\
\hline$\beta$ & $3.08 / 2.72$ & 37.23 \\
\hline$\gamma$ & - & 137.68 \\
\hline$\delta$ & 7.24 & 129.10 \\
\hline$\varepsilon$ & 7.24 & 127.97 \\
\hline$\zeta$ & 7.18 & 126.26 \\
\hline$C^{\prime}$ & - & $170.95(b)$ \\
\hline
\end{tabular}




\begin{tabular}{|c|c|c|}
\hline Arg-10 & & - \\
\hline $\mathrm{NH}$ & 8.13 & 51.46 \\
\hline$\alpha$ & 4.20 & 28.09 \\
\hline$\beta$ & $1.79 / 1.64$ & 25.06 \\
\hline$\gamma$ & 1.51 & 40.27 \\
\hline$\delta$ & 3.12 & - \\
\hline$\varepsilon$ & 7.56 & 156.65 \\
\hline$\zeta$ & - & 173.08 \\
\hline $\mathrm{C}^{\prime}$ & - & \\
\hline
\end{tabular}

(a) Could not be assigned

(b) Might be interchanged

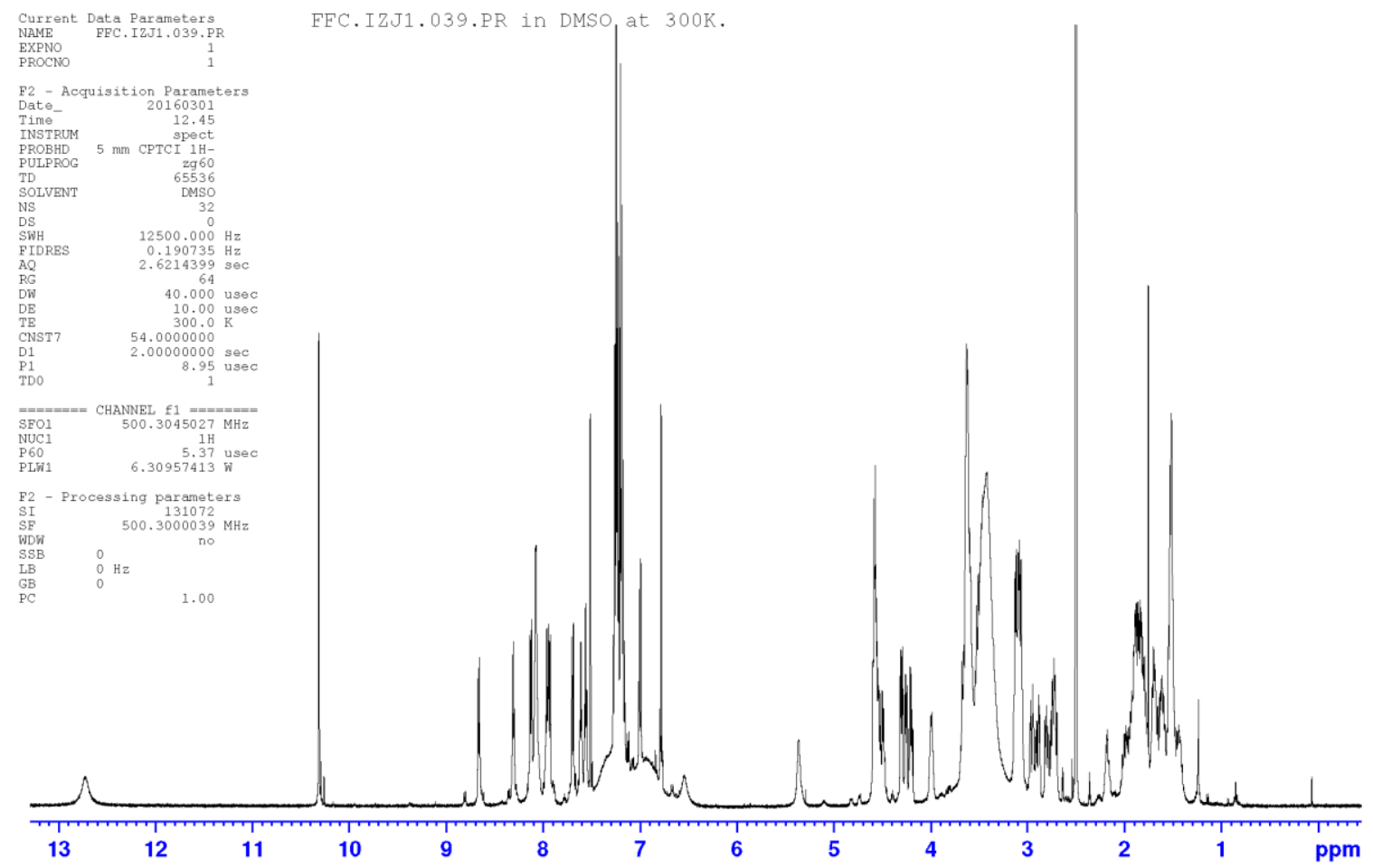




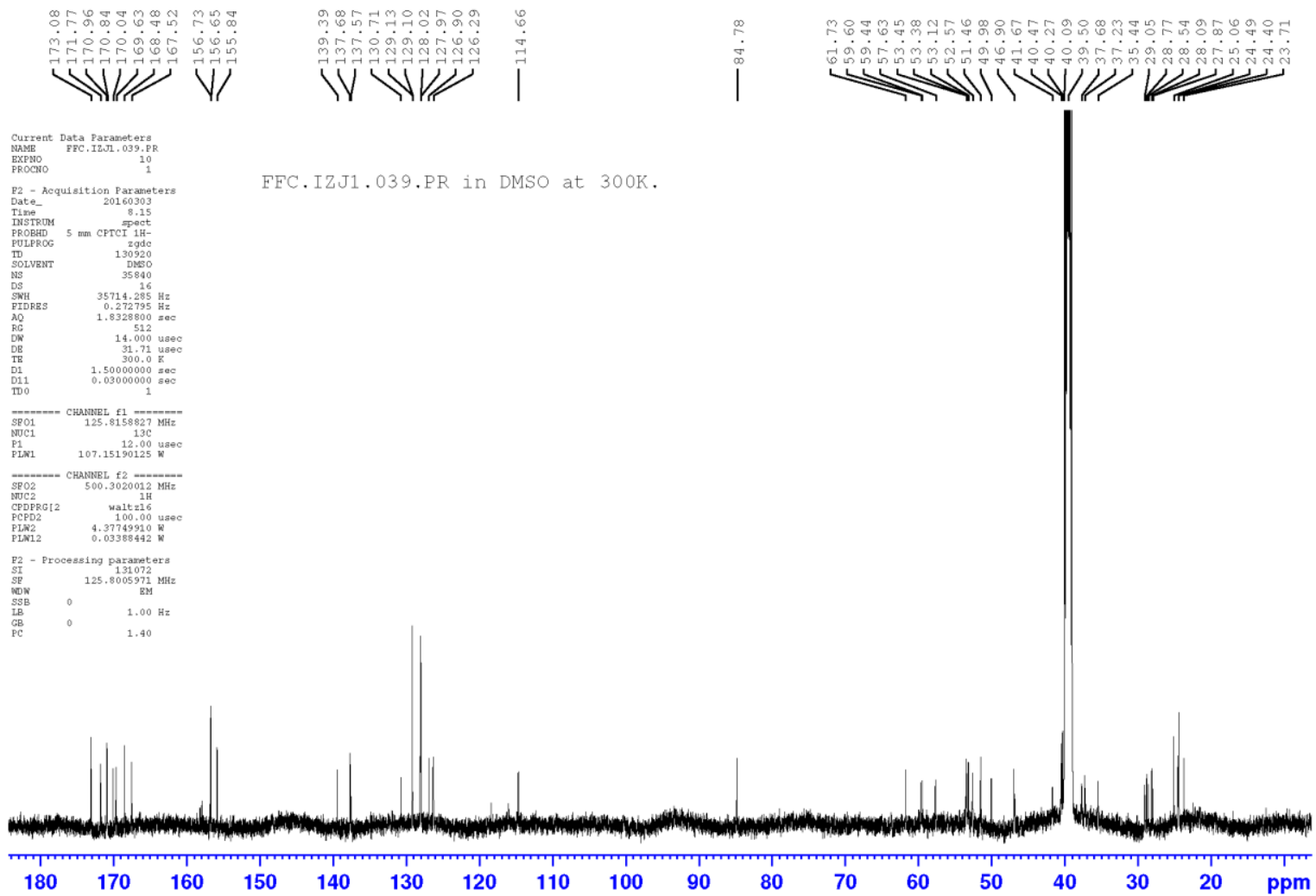


[mono-lodo]-Angiotensin III

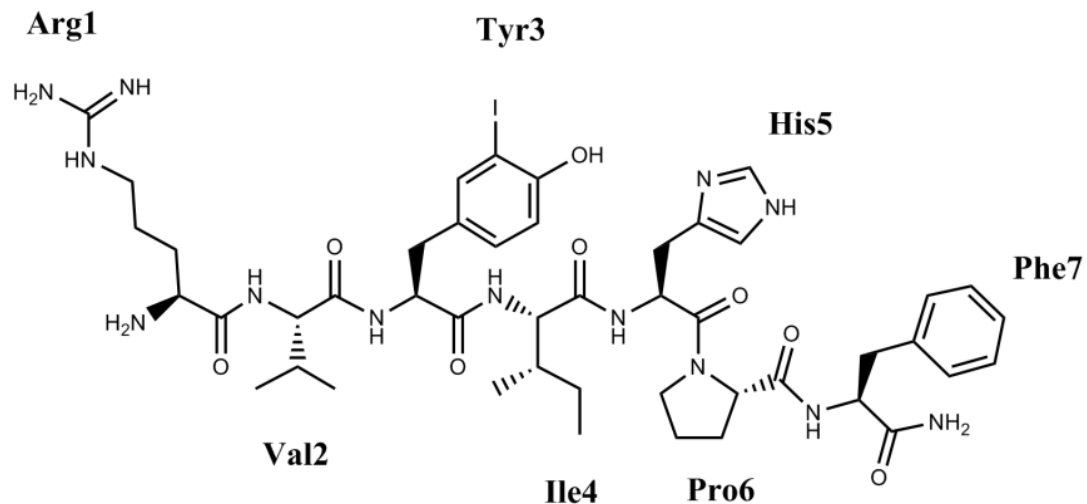

${ }^{1} \mathrm{H}$ NMR (500 MHz, $\mathrm{d}_{6}$-DMSO, $300 \mathrm{~K}$ ) and ${ }^{13} \mathrm{C}$ NMR (125 MHz, $\mathrm{d}_{6}$-DMSO, $300 \mathrm{~K}$ ).

\begin{tabular}{|c|c|c|}
\hline & ${ }^{1} \mathrm{H}$ & ${ }^{13} \mathrm{C}$ \\
\hline \multicolumn{3}{|l|}{ Arg-1 } \\
\hline $\mathrm{NH}_{3}{ }^{+}$ & 8.12 & - \\
\hline$\alpha$ & 3.87 & 51.72 \\
\hline$\beta$ & 1.64 & 28.62 \\
\hline$\gamma$ & 1.46 & 24.28 \\
\hline$\delta$ & 3.09 & 40.12 \\
\hline$\varepsilon$ & 7.60 & - \\
\hline$\zeta$ & - & 156.71 \\
\hline$C^{\prime}$ & - & 168.22 \\
\hline \multicolumn{3}{|l|}{ Val-2 } \\
\hline $\mathrm{NH}$ & 8.35 & - \\
\hline$\alpha$ & 4.27 & 57.12 \\
\hline$\beta$ & 2.02 & 31.11 \\
\hline$\gamma$ & 0.84 & 19.19 \\
\hline$\gamma^{\prime}$ & 0.79 & 17.57 \\
\hline
\end{tabular}




\begin{tabular}{|c|c|c|}
\hline$C^{\prime}$ & - & 170.48 \\
\hline \multicolumn{3}{|l|}{ Tyr-3 } \\
\hline $\mathrm{NH}$ & 8.10 & - \\
\hline$\alpha$ & 4.50 & 53.93 \\
\hline$\beta$ & $2.77 / 2.59$ & 35.77 \\
\hline$\gamma$ & - & 130.29 \\
\hline$\delta 1$ & 7.60 & 138.97 \\
\hline$\delta 2$ & 7.09 & 130.36 \\
\hline$\varepsilon 1$ & - & 84.27 \\
\hline$\varepsilon 2$ & 6.75 & 114.41 \\
\hline$\zeta$ & - & 155.02 \\
\hline$\zeta-\mathrm{OH}$ & 10.17 & - \\
\hline$C^{\prime}$ & - & 170.97 \\
\hline \multicolumn{3}{|l|}{ Ile-4 } \\
\hline $\mathrm{NH}$ & 8.00 & - \\
\hline$\alpha$ & 4.15 & 56.65 \\
\hline$\beta$ & 1.66 & 36.62 \\
\hline$\beta-\mathrm{Me}$ & 0.75 & 15.19 \\
\hline$\gamma$ & $1.36 / 1.05$ & 24.10 \\
\hline$\delta$ & 0.77 & 10.85 \\
\hline$C^{\prime}$ & - & 170.85 \\
\hline \multicolumn{3}{|l|}{ His-5 } \\
\hline $\mathrm{NH}$ & 8.41 & - \\
\hline$\alpha$ & 4.79 & $\sim 47.7$ (broad) \\
\hline$\beta$ & $3.05 / 2.93$ & $\sim 26.3$ (broad) \\
\hline$\gamma$ & - & (a) \\
\hline
\end{tabular}




\begin{tabular}{|c|c|c|}
\hline$\delta$ & 7.38 (broad) & 117.1 (broad) \\
\hline$\varepsilon$ & $\sim 8.9$ (very broad) & 133.8 (broad) \\
\hline$\varepsilon-\mathrm{NH}_{2}{ }^{+}$ & $\sim 14.2$ (broad) & - \\
\hline $\mathrm{C}^{\prime}$ & - & (a) \\
\hline \multicolumn{3}{|l|}{ Pro-3 } \\
\hline$\alpha$ & 4.26 & 59.87 \\
\hline$\beta$ & $1.98 / 1.69$ & 29.13 \\
\hline$\gamma$ & 1.77 & 24.25 \\
\hline$\delta$ & $3.63 / 3.46$ & 46.99 \\
\hline$C^{\prime}$ & - & 171.43 \\
\hline \multicolumn{3}{|l|}{ Phe-9 } \\
\hline $\mathrm{NH}$ & 8.01 (very broad) & - \\
\hline$\alpha$ & 4.38 & 53.83 \\
\hline$\beta$ & $3.05 / 2.85$ & 37.01 \\
\hline$\gamma$ & - & 137.88 \\
\hline$\delta$ & 7.23 & 129.17 \\
\hline$\varepsilon$ & 7.23 & 128.00 \\
\hline$\zeta$ & 7.17 & 126.20 \\
\hline $\mathrm{C}^{\prime}$ & - & 172.55 \\
\hline $\mathrm{NH}_{2}$ & $7.26 / 7.09$ & - \\
\hline
\end{tabular}

(a) Could not be assigned due to extreme line broadening 


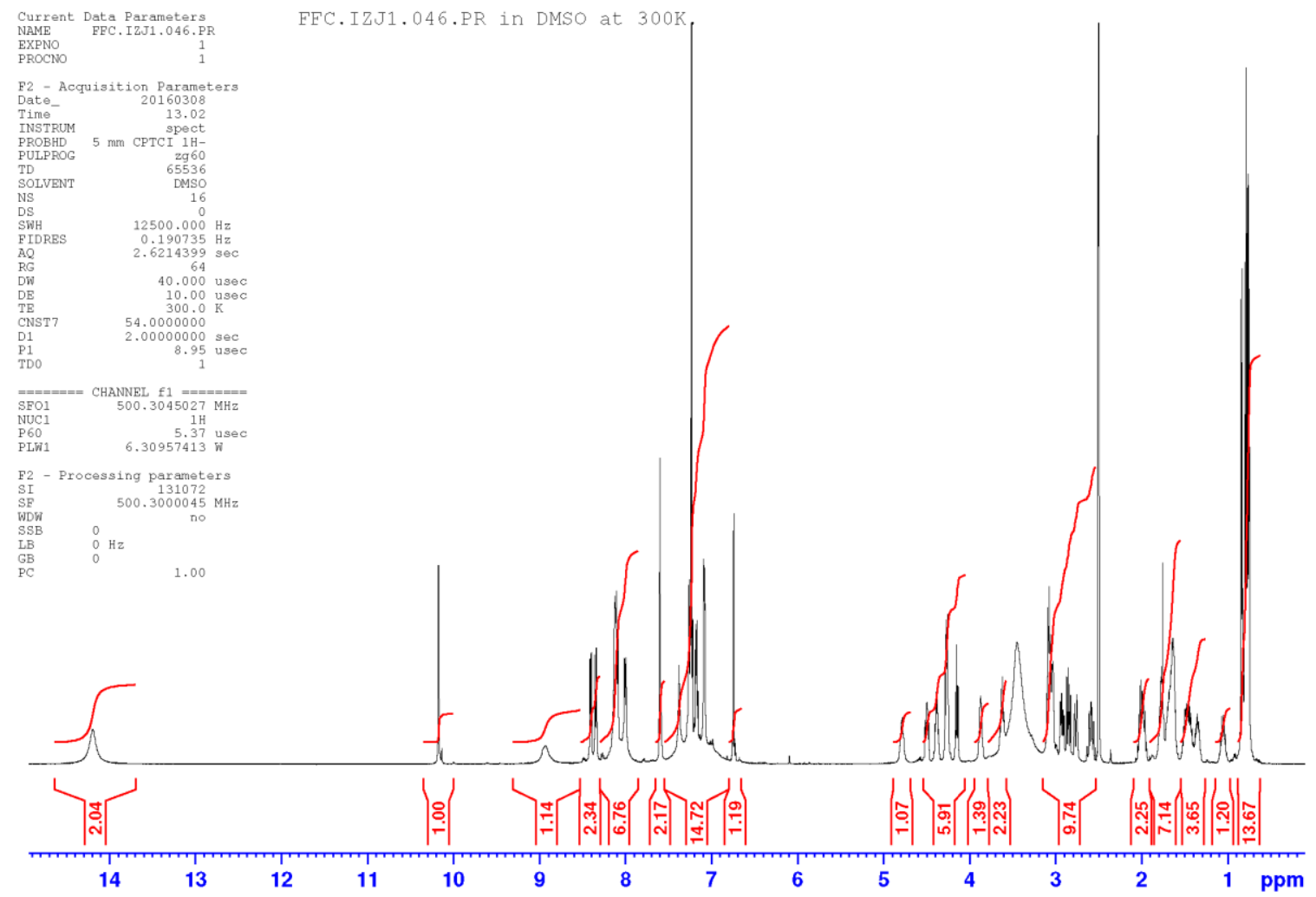




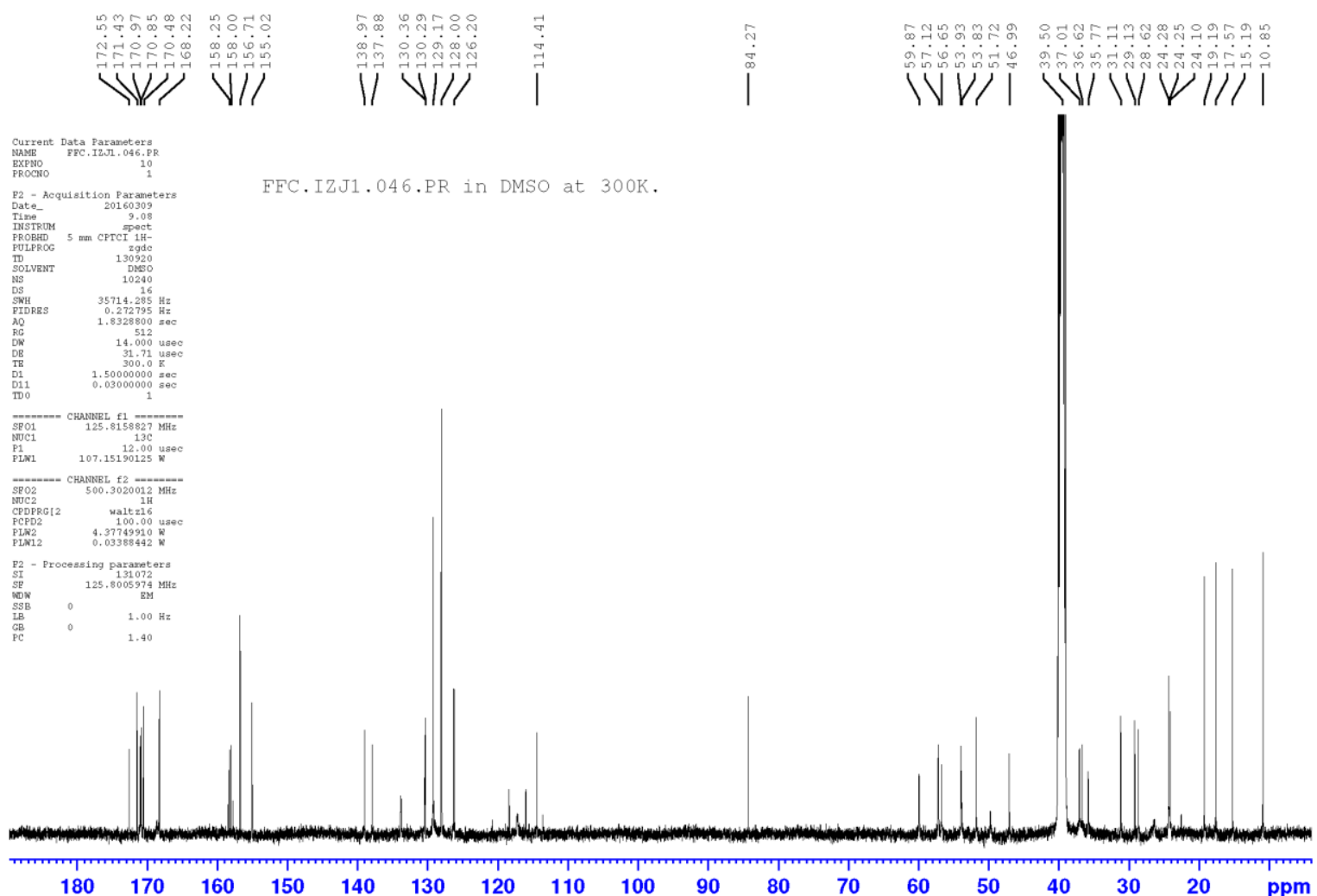


[mono-lodo]-ACP fragment 65-74

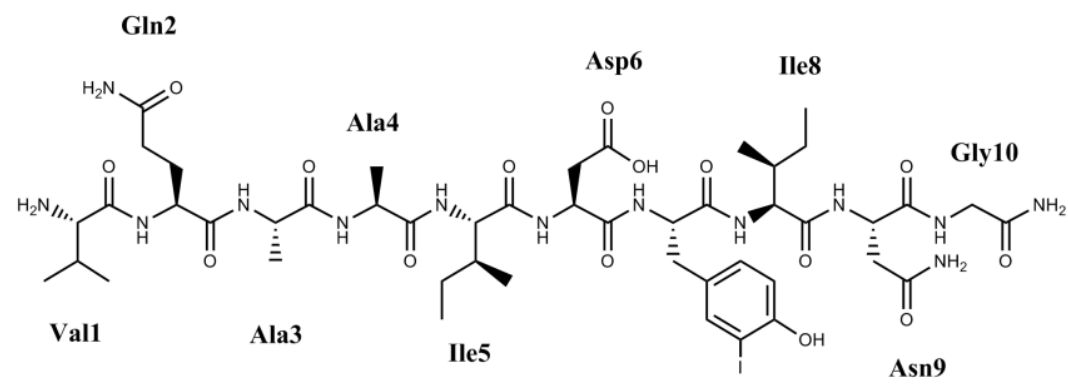

Tyr7

${ }^{1} \mathrm{H}$ NMR (500 MHz, $\mathrm{d}_{6}$-DMSO, $295 \mathrm{~K}$ ) and ${ }^{13} \mathrm{C}$ NMR (125 MHz, $\mathrm{d}_{6}$-DMSO, $295 \mathrm{~K}$ ).

\begin{tabular}{|c|c|c|}
\hline & ${ }^{1} \mathrm{H}$ & ${ }^{13} \mathrm{C}$ \\
\hline $\mathrm{NH}_{3}{ }^{+}$ & 8.04 & - \\
\hline$\alpha$ & 3.60 & 57.25 \\
\hline$\beta$ & 2.03 & 29.89 \\
\hline$\gamma$ & 0.92 & 18.33 \\
\hline$\gamma^{\prime}$ & 0.91 & 17.70 \\
\hline$C^{\prime}$ & - & 167.63 \\
\hline Gln-2 & 8.53 & - \\
\hline$\alpha$ & 4.33 & 52.07 \\
\hline$\beta$ & $1.87 / 1.76$ & 28.02 \\
\hline$\gamma$ & 2.13 & 31.29 \\
\hline$\delta$ & - & 173.73 \\
\hline$\delta-\mathrm{NH}_{2}$ & $7.29 / 6.84$ & - \\
\hline$C^{\prime}$ & - & 170.29 \\
\hline Ala-3 & 8.18 & - \\
\hline$\alpha$ & 4.28 & 47.97 \\
\hline$\beta$ & 1.18 & 18.22 \\
\hline$C^{\prime}$ & - & 171.86 \\
\hline
\end{tabular}




\begin{tabular}{|c|c|c|}
\hline Ala-4 & 8.11 & - \\
\hline$\alpha$ & 4.32 & 48.02 \\
\hline$\beta$ & 1.17 & 17.79 \\
\hline$C^{\prime}$ & - & 171.91 \\
\hline Ile-5 & 7.69 & - \\
\hline$\alpha$ & 4.13 & 56.60 \\
\hline$\beta$ & 1.65 & 36.99 \\
\hline$\beta-\mathrm{Me}$ & 0.73 & 15.27 \\
\hline$\gamma$ & $1.35 / 1.00$ & 24.01 \\
\hline$\delta$ & 0.76 & 11.11 \\
\hline$C^{\prime}$ & - & 170.60 \\
\hline Asp-6 & 8.17 & - \\
\hline$\alpha$ & 4.51 & 49.54 \\
\hline$\beta$ & $2.62 / 2.43$ & 36.27 \\
\hline$\gamma$ & - & 171.65 \\
\hline$C^{\prime}$ & - & 170.47 \\
\hline
\end{tabular}

The amide protons of Ala3 and Asp6 overlap at 300K. 


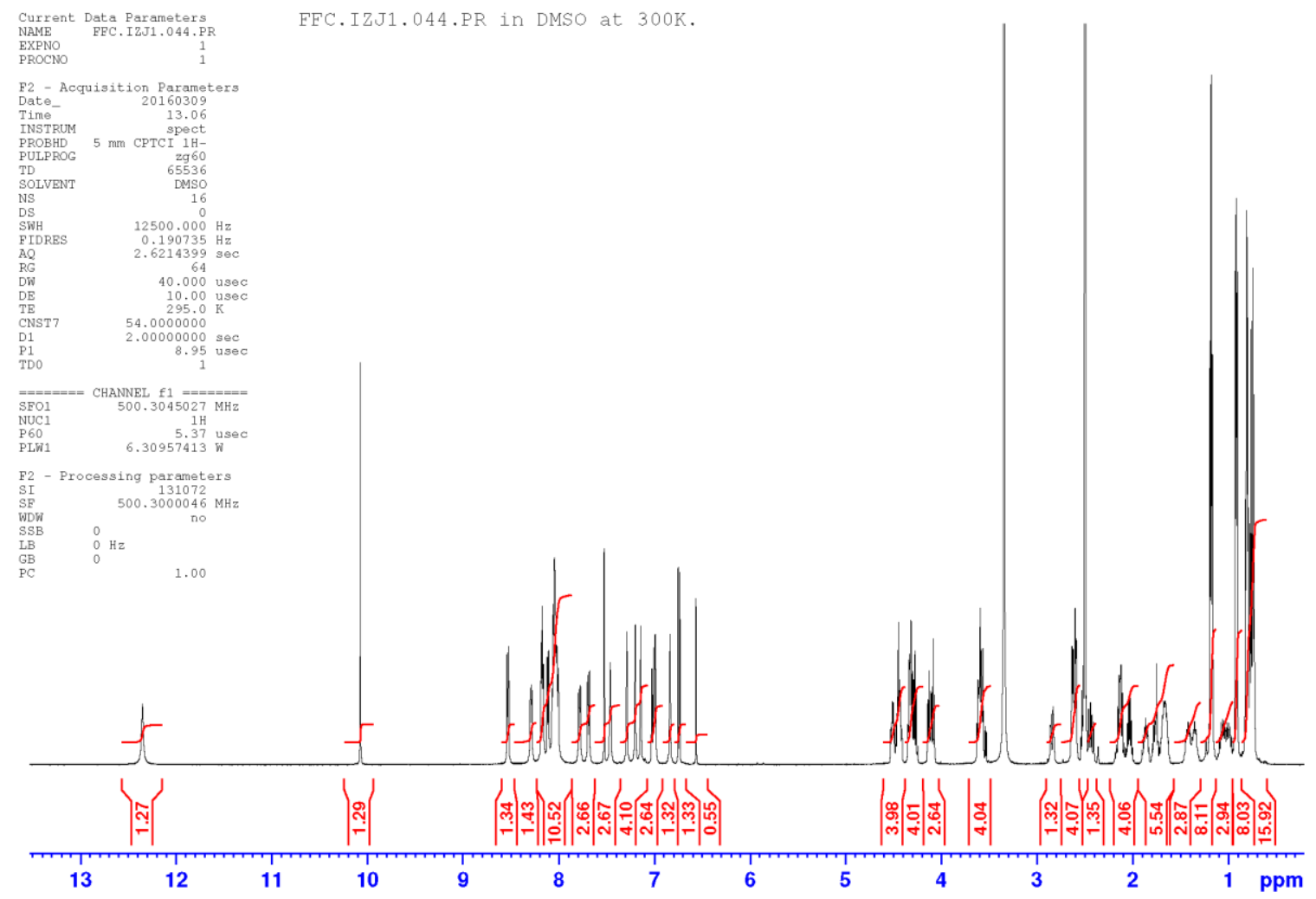



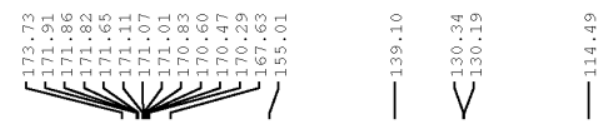

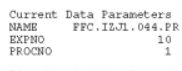

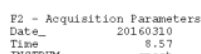

FFC.IZJ1.044.PR in DMSO at $300 \mathrm{~K}$.

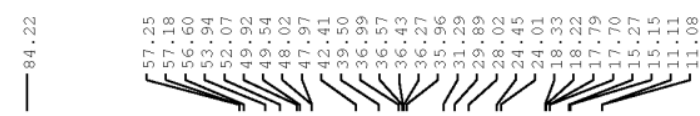

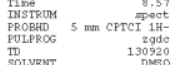

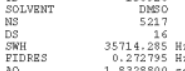

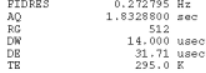

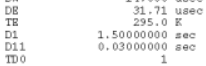

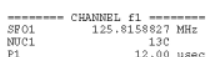

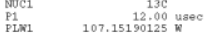

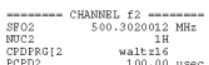

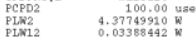

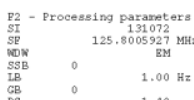

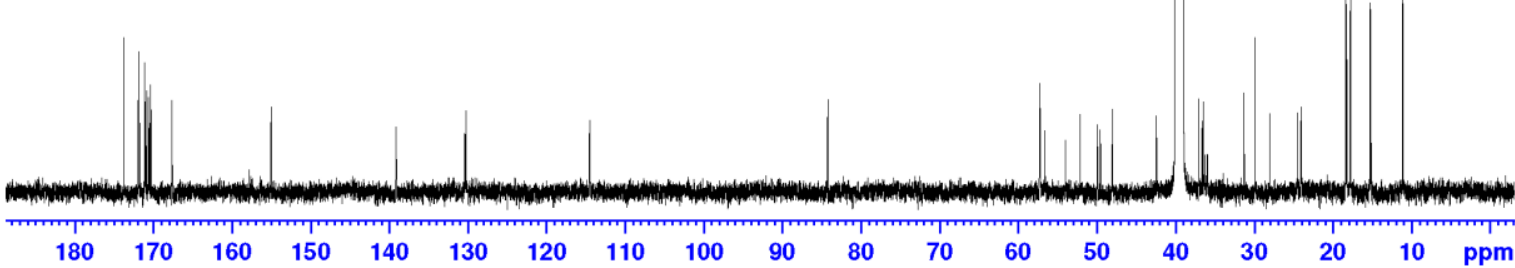


[mono-lodo-Tyr ${ }^{8}$ ]-Substance P

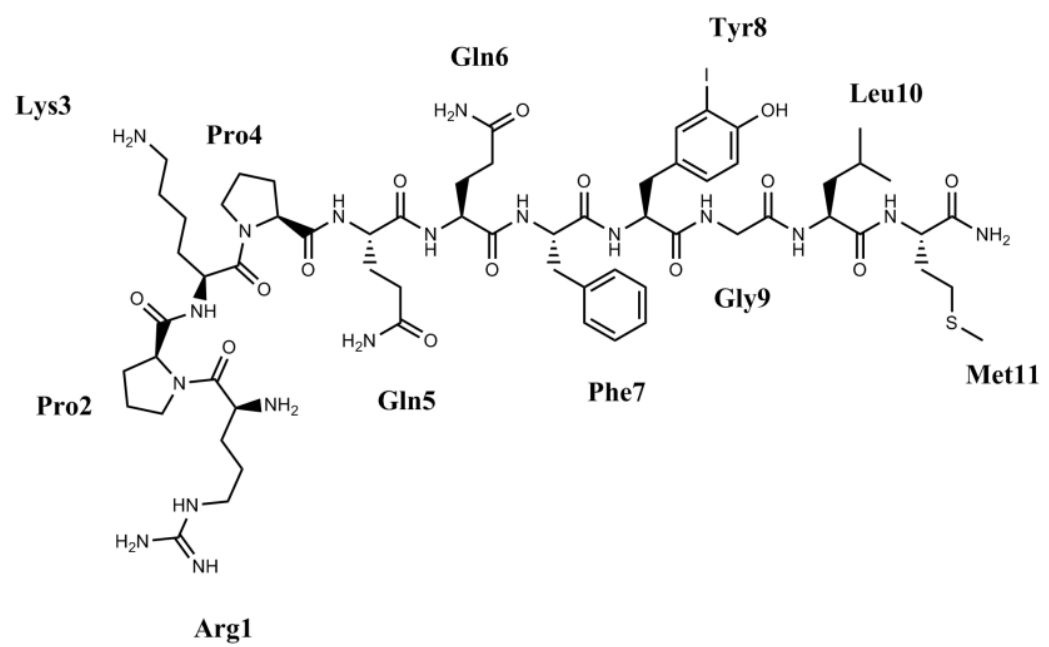

${ }^{1} \mathrm{H}$ NMR (700 MHz, $\mathrm{d}_{6}$-DMSO, $295 \mathrm{~K}$ ) and ${ }^{13} \mathrm{C}$ NMR (175 MHz, $\mathrm{d}_{6}$-DMSO, $295 \mathrm{~K}$ ).

\begin{tabular}{|c|c|c|}
\hline & ${ }^{1} \mathrm{H}$ & ${ }^{13} \mathrm{C}$ \\
\hline Arg-1 & 8.18 & - \\
\hline$\alpha$ & 4.16 & 50.48 (a) \\
\hline$\beta$ & $1.76 / 1.70$ & 27.11 \\
\hline$\gamma$ & 1.62 & 23.62 \\
\hline$\delta$ & 3.13 & 40.22 \\
\hline$\varepsilon$ & 7.69 & - \\
\hline$\zeta$ & - & 156.79 \\
\hline$C^{\prime}$ & - & 166.84 \\
\hline Pro-2 & 4.43 & 59.24 \\
\hline$\beta$ & $2.11 / 1.75$ & 29.22 \\
\hline$\gamma$ & $1.91 / 1.83$ & 24.66 \\
\hline$\delta$ & $3.70 / 3.46$ & 47.04 \\
\hline$C^{\prime}$ & - & 170.98 \\
\hline Lys-3 & 8.31 & - \\
\hline$\alpha$ & 4.43 & 50.43 (a) \\
\hline
\end{tabular}




\begin{tabular}{|c|c|c|}
\hline$\beta$ & $1.70 / 1.51$ & 30.12 \\
\hline$\gamma$ & 1.39 & 21.84 \\
\hline$\delta$ & 1.54 & 26.56 \\
\hline$\varepsilon$ & 2.75 & 38.58 \\
\hline$\zeta$ & 7.77 & - \\
\hline$C^{\prime}$ & - & 170.38 \\
\hline Pro-4 & 4.30 & 59.83 \\
\hline$\beta$ & $2.09 / 1.82$ & 29.03 \\
\hline$\gamma$ & $1.92 / 1.85$ & 24.66 \\
\hline$\delta$ & $3.60 / 3.57$ & 46.88 \\
\hline$C^{\prime}$ & - & 172.04 \\
\hline Gln-5 & 8.27 & - \\
\hline$\alpha$ & 4.12 & 52.80 \\
\hline$\beta$ & $1.85 / 1.73$ & 27.39 \\
\hline$\gamma$ & 2.12 & 31.48 \\
\hline$\delta$ & - & 174.24 \\
\hline $\mathrm{NH}_{2}$ & $7.32 / 6.87$ & - \\
\hline$C^{\prime}$ & - & 174.24 \\
\hline Gln-6 & 7.85 & - \\
\hline$\alpha$ & 4.15 & 52.30 \\
\hline$\beta$ & $1.81 / 1.71$ & 27.90 \\
\hline$\gamma$ & 2.04 & 31.57 \\
\hline$\delta$ & - & 174.19 \\
\hline $\mathrm{NH}_{2}$ & $7.33 / 6.87$ & - \\
\hline$C^{\prime}$ & - & 170.87 \\
\hline Phe-7 & 7.97 & - \\
\hline
\end{tabular}




\begin{tabular}{|c|c|c|}
\hline$\alpha$ & 4.42 & 54.13 \\
\hline$\beta$ & $2.93 / 2.74$ & 37.50 \\
\hline$\gamma$ & - & 137.59 \\
\hline$\delta$ & 7.15 & 129.11 \\
\hline$\varepsilon$ & 7.19 & 128.04 \\
\hline$\zeta$ & 7.14 & 126.20 \\
\hline $\mathrm{C}^{\prime}$ & - & 170.92 \\
\hline Tyr8 & 8.03 & - \\
\hline$\alpha$ & 4.42 & 54.29 \\
\hline$\beta$ & $2.90 / 2.67$ & 36.18 \\
\hline$\gamma$ & - & 130.22 \\
\hline$\delta 1$ & 7.58 & 139.21 \\
\hline$\delta 2$ & 7.08 & 130.33 \\
\hline$\varepsilon 1$ & - & 84.28 \\
\hline$\varepsilon 2$ & 6.77 & 114.63 \\
\hline$\zeta$ & - & 155.10 \\
\hline$\zeta-\mathrm{OH}$ & 10.09 & - \\
\hline $\mathrm{C}^{\prime}$ & - & 171.30 \\
\hline Gly-9 & 8.23 & - \\
\hline$\alpha$ & 3.73 & 41.99 \\
\hline $\mathrm{C}^{\prime}$ & - & 168.64 \\
\hline Leu-10 & 8.02 & - \\
\hline$\alpha$ & 4.29 & 51.21 \\
\hline$\beta$ & 1.47 & 40.70 \\
\hline$\gamma$ & 1.61 & 24.12 \\
\hline$\delta$ & 0.88 & 23.13 \\
\hline
\end{tabular}




\begin{tabular}{|c|c|c|}
\hline$\overline{\delta^{\prime}}$ & 0.84 & 21.59 \\
\hline$C^{\prime}$ & - & 171.93 \\
\hline Met-11 & 7.98 & - \\
\hline$\alpha$ & 4.23 & 51.76 \\
\hline$\beta$ & $1.92 / 1.81$ & 31.59 \\
\hline$\gamma$ & $2.45 / 2.38$ & 29.70 \\
\hline$\delta$ & 2.02 & 14.65 \\
\hline$C^{\prime}$ & - & 173.08 \\
\hline $\mathrm{NH}_{2}$ & $7.21 / 7.07$ & - \\
\hline
\end{tabular}

(a) Might be interchanged

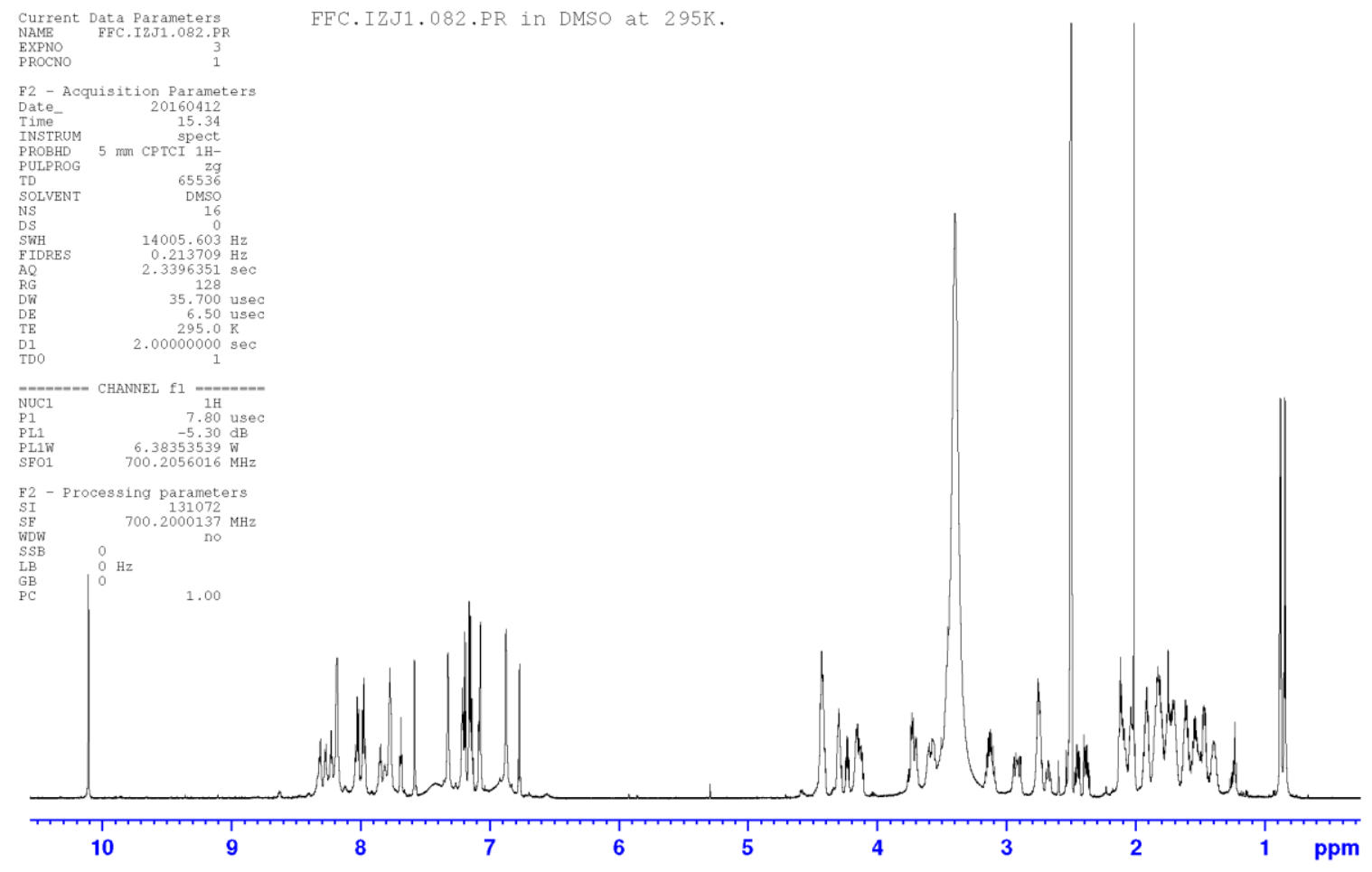




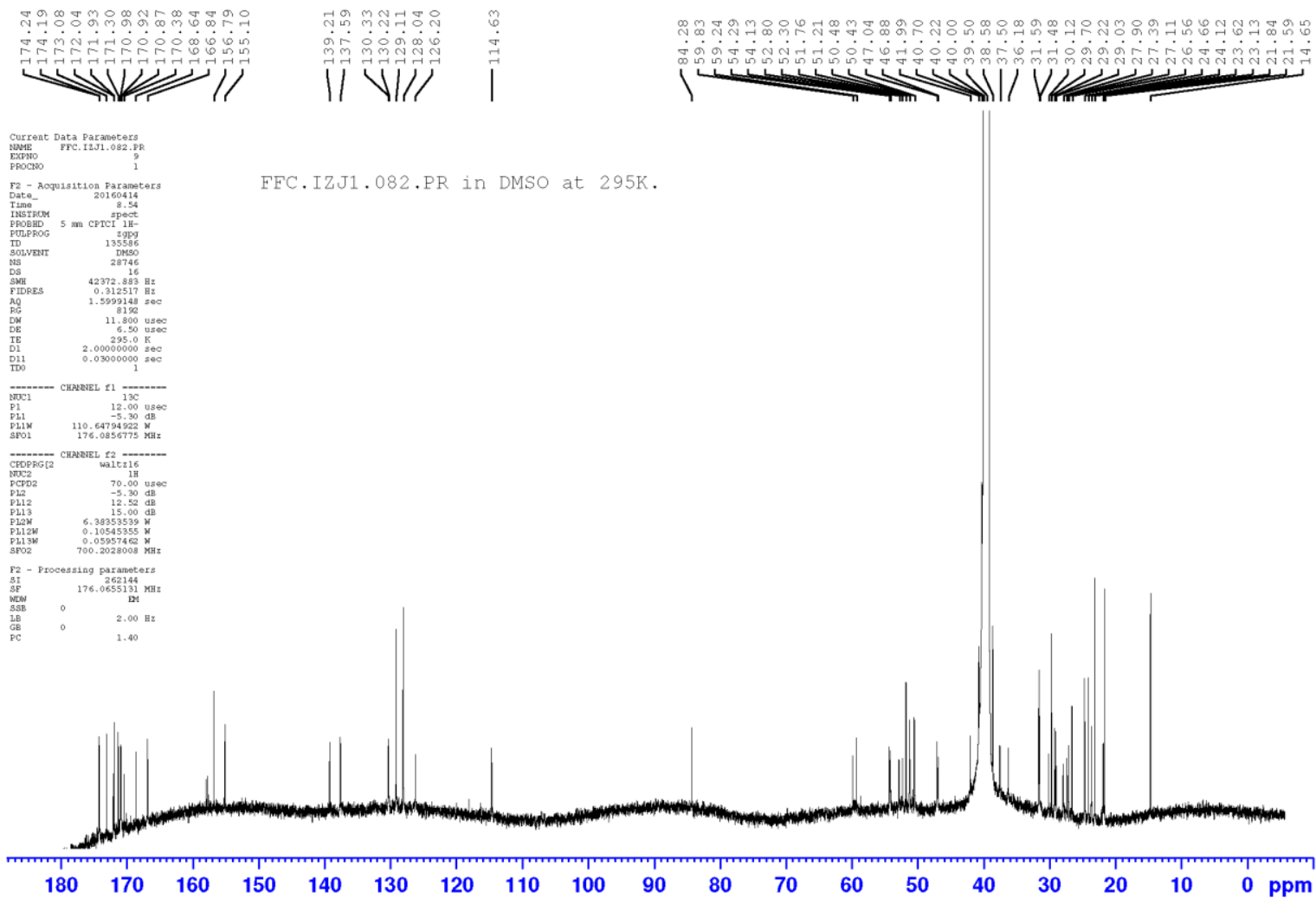

\section{Supporting References}

1. J. M. Collins, K. A. Porter, S. K. Singh and G. S. Vanier, Organic letters, 2014, 16, 940-943.

2. J. M. Chalker, C. S. Wood and B. G. Davis, J. Am. Chem. Soc., 2009, 131, 16346-16347.

3. V. Hong, S. I. Presolski, C. Ma and M. Finn, Angewandte Chemie International Edition, 2009, 48, 9879-9883. 